\title{
G0.253+0.016: A centrally condensed, high-mass protocluster
}

\author{
J. M. Rathborne \\ CSIRO Astronomy and Space Science, P.O. Box 76, Epping NSW, 1710, Australia; \\ Jill.Rathborne@csiro.au \\ and \\ S. N. Longmore ${ }^{1}$ \\ European Southern Observatory, Karl-Schwarzschild-Str. 2, 85748 Garching bei Munchen, \\ Germany \\ and \\ J. M. Jackson \\ Institute for Astrophysical Research, Boston University, Boston, MA 02215, USA \\ and \\ J. B. Foster ${ }^{2}$ \\ Institute for Astrophysical Research, Boston University, Boston, MA 02215, USA \\ and \\ Y. Contreras \\ CSIRO Astronomy and Space Science, P.O. Box 76, Epping NSW, 1710, Australia \\ and \\ G. Garay \\ Universidad de Chile, Camino El Observatorio1515, Las Condes, Santiago, Chile \\ and \\ L. Testi \\ European Southern Observatory, Karl-Schwarzschild-Str. 2, 85748 Garching bei Munchen, \\ Germany; INAF-Arcetri, Largo E. Fermi 5, I-50125 Firenze, Italy; Excellence Cluster \\ Universe, Boltzmannstr. 2, D-85748, Garching, Germany
}


and

J. F. Alves

University of Vienna, Türkenschanzstrasse 17, 1180 Vienna, Austria

and

J. Bally

Center for Astrophysics and Space Astronomy, University of Colorado, UCB 389, Boulder, CO 8030

and

N. Bastian

Astrophysics Research Institute, Liverpool John Moores University, Egerton Wharf, Birkenhead CH41 1LD, UK

and

J. M. D. Kruijssen

Max-Planck Institut fur Astrophysik, Karl-Schwarzschild-Strasse 1, 85748, Garching, Germany

and

E. Bressert

CSIRO Astronomy and Space Science, P.O. Box 76, Epping NSW, 1710, Australia

\begin{abstract}
Despite their importance as stellar nurseries and the building blocks of galaxies, very little is known about the formation of the highest mass clusters. The dense clump G0.253+0.016 represents an example of a clump that may form an Arches-like, high-mass cluster. Here we present molecular line maps toward G0.253+0.016 taken as part of the MALT90 molecular line survey, complemented with APEX observations. Combined, these data reveal the global physical properties and kinematics of G0.253+0.016. Recent Herschel data show that while the dust temperature is low $(\sim 19 \mathrm{~K})$ toward its centre, the dust temperature
\end{abstract}


on the exterior is higher $(\sim 27 \mathrm{~K})$ due to external heating. Our new molecular line data reveal that, overall, the morphology of dense gas detected toward G0.253+0.016 matches very well its IR extinction and dust continuum emission. An anti-correlation between the dust and gas column densities toward its centre indicates that the clump is centrally condensed with a cold, dense interior in which the molecular gas is chemically depleted. The velocity field shows a strong gradient along the clump's major axis, with the blue-shifted side at higher Galactic longitude. The optically thick gas tracers are systematically red-shifted with respect to the optically thin and hot gas tracers, indicating radial motions. The gas kinematics and line ratios support the recently proposed scenario in which G0.253+0.016 results from a tidal compression during a recent pericentre passage near SgrA*. Because G0.253+0.016 represents an excellent example of a clump that may form a high-mass cluster, its detailed study should reveal a wealth of knowledge about the early stages of cluster formation.

Subject headings: dust, extinction-stars:formation-ISM:clouds-infrared:ISM-radio lines:ISM

\section{Introduction}

G0.253+0.016, an infrared dark cloud (IRDC) located in close proximity to the Galactic Centre (Lis \& Carlstrom 1994; Lis \& Menten 1998; Lis et al. 2001; Immer et al. 2012), has recently gained attention as a potential candidate of a high-mass cluster in a very early stage of formation (Longmore et al. 2012). G0.253+0.016 is clearly seen as an extinction feature from mid- to far-IR wavelengths. Recent modelling of Herschel data reveals a central dust temperature of $\sim 20 \mathrm{~K}$, peak $\mathrm{H}_{2}$ column density of $\sim 3.3 \times 10^{23} \mathrm{~cm}^{-2}$, and mass of $\sim 1.3 \times 10^{5} \mathrm{M}_{\odot}$ (Molinari et al. 2011; Longmore et al. 2012). Despite its high mass and density, it shows little evidence for wide-spread star formation, consistent with the low dust temperature. This combination of properties, little star formation and a low dust temperature, yet with a high mass and column density, make G0.253+0.016 extreme when compared to other known Galactic molecular clumps. Indeed, Ginsburg et al. (2012) find no

\footnotetext{
${ }^{1}$ Current Address: Astrophysics Research Institute, Liverpool John Moores University, Egerton Wharf, Birkenhead CH41 1LD, UK

${ }^{2}$ Current Address: Department of Astronomy, Yale University, P.O. Box 208101 New Haven, CT 065208101, USA
} 
other examples of cold, starless clumps with masses $>10^{4} \mathrm{M}_{\odot}$ in the recent Bolocam Galactic Plane Survey. Because it contains $\sim 10^{5} \mathrm{M}_{\odot}$ of material, G0.253+0.016 has sufficient mass to form a young massive cluster (YMC) through direct collapse. As such, its detailed study may reveal the initial conditions within a protocluster and the processes by which a high-mass cluster is formed.

Young massive clusters are gravitationally bound stellar systems with masses $>10^{4} \mathrm{M}_{\odot}$ and ages < 100 Myr (Portegies Zwart et al. 2010). Only a few YMCs have been identified within our Galaxy (e.g., Arches, Quintuplet, Westerlund 1, RSCG, GLIMPSE-CO1: Figer et al. 1999; Clark et al. 2005; Figer et al. 2006; Davies et al. 2011). Recent work suggests that YMCs may be the 'missing link' between open clusters and globular clusters and, as such, may be the local-universe analogs of the progenitors of globular clusters (e.g. Elmegreen \& Efremov 1997; Bastian 2008; Kruijssen \& Cooper 2012). Characterising and understanding how these YMCs form is critical to connect Galactic and extra-galactic cluster formation. Identifying a sample of Galactic molecular clouds that may form YMCs is important because their detailed study can shed light not only on how these high-mass clusters form, but potentially on how all clusters form. To date, G0.253+0.016 is one of a handful of known molecular clumps with enough mass to form a cluster of similar mass to these Galactic YMCs through direct collapse. Because G0.253+0.016 shows evidence for structure on small spatial scales $\left(7.5^{\prime \prime}\right)$, Longmore et al. (2012) speculate that it may be destined to form a YMC through hierarchical fragmentation, in a scaled up version of open cluster formation. Indeed, recent models predict that G0.253+0.016 should form a cluster through hierarchical fragmentation in which $>80 \%$ of the stars should remain bound after the expulsion of the residual gas by feedback (Kruijssen 2012).

Located at a distance of $\sim 8.5 \mathrm{kpc}, \mathrm{G} 0.253+0.016$ lies $\sim 100 \mathrm{pc}$ from the Galactic Centre (GC; Molinari et al. 2011). Its location within the harsh GC environment may provide clues to the formation of such a high-mass protocluster and whether star formation can progress within it. What remains unknown is if the influence of strong tidal forces, high turbulence, and extreme magnetic fields, radiation fields, and cosmic ray fluxes within the GC region helps or hinders the formation of a high-mass cluster. While some YMCs within the Galaxy are located close to the GC (e.g. Arches, Quintuplet), recent work finds that given the large reservoir of dense gas available, the broader GC region appears to be under-producing stars compared to commonly assumed relations between gas mass and star formation (e.g., Longmore et al. 2013a).

Molecular line studies show that the bulk of the gas in the Central Molecular Zone (CMZ), the region within $\sim 200 \mathrm{pc}$ of the Galactic Centre, has temperatures of $\sim 80 \mathrm{~K}$ and densities $>10^{4} \mathrm{~cm}^{-3}$ (Walmsley et al. 1986; Ao et al. 2013). Somewhat more surprising is 
that many complex organic molecules show bright, widespread emission across the CMZ, leading to the speculation that shock chemistry might dominate in the region (Wilson et al. 1982; Martín-Pintado et al. 2001). Molecular clouds located in the CMZ have exceptional physical properties; they are denser, warmer, more turbulent, and more massive compared to molecular clouds in the Galactic disk. Such high densities are required for the molecular clouds to survive in the steep Galactic Center gravitational potential (Bania et al. 1986).

Previous molecular line observations toward G0.253+0.016 (also known as GCM0.25+0.011 and M0.25+0.01; Guesten et al. 1981; Lis \& Carlstrom 1994) reveal large line-widths, indicating a high degree of turbulence, similar to other molecular clouds near the GC. Given its location in the CMZ where the interstellar radiation field (ISRF) and cosmic ray ionisation rate (CRIR) are high (Clark et al. 2013; Yusef-Zadeh et al. 2013), one might expect a high-density clump like G0.253+0.016 to be externally heated. Indeed, observations have shown that it has a low internal luminosity and that its derived dust temperature increases smoothly from $\sim 19 \mathrm{~K}$ in its centre to $\sim 27 \mathrm{~K}$ toward its edges (Lis et al. 2001; Longmore et al. 2012).

These observations are consistent with recent SPH modelling of the dust and gas temperature distribution in G0.253+0.016 (Clark et al. 2013). In these models, the gas and dust temperatures are derived for G0.253+0.016 as the ISRF and CRIR vary. This modelling suggest that a very high ISRF, combined with a high CRIR, reproduces well the observed discrepant dust and gas temperatures: both the ISRF and CRIR are predicted to be 1000 times higher than the values measured in the solar neighbourhood. Indeed, the models of Clark et al. (2013) find that the gas and dust are not coupled, have different temperatures throughout the clump, and that G0.253+0.016 is externally heated with a relatively cooler interior that is highly sub-structured.

Despite its high mass, there appears to be very little active high-mass star formation within G0.253+0.016. The detection of a weak water maser toward it (Lis \& Carlstrom 1994; Breen \& Ellingsen 2011) supports the idea that star formation may be 'turning on' within this clump (Lis \& Menten 1998). JVLA radio continuum observations reveal three compact $\mathrm{H}$ is regions located toward the periphery of the clump; their fluxes suggest that if they lie at the distance of G0.253+0.016, then they are powered by B0.5 ZAMS stars (Rodríguez \& Zapata 2013). These H II regions, however, have not yet been definitively shown to be associated with G0.253+0.016. Recent CARMA and SMA observations of $\mathrm{N}_{2} \mathrm{H}^{+}$line and dust continuum emission show little evidence for dense material on small scales, leading Kauffmann et al. (2013) to speculate that G0.253+0.016 may lack the potential to form a cluster.

A measured gas temperature of $\sim 80 \mathrm{~K}$, significantly higher than its derived dust tem- 
perature $(\sim 20 \mathrm{~K})$, led Lis et al. (2001) to speculate that shocks associated with clump-clump collisions might be the dominant heating source for the gas, rather than reprocessed UV radiation. Indeed, recent $\mathrm{H}_{2} \mathrm{CO}$ mapping of the dense gas across the $\mathrm{CMZ}$ measure gas kinetic temperatures ranging from 50-100 K (Ao et al. 2013), leading to the conclusion that the gas may be heated by the dissipation of turbulent energy and/or cosmic rays rather than by photon heating. This idea is also supported by other recent work that suggests that the high cosmic ray ionisation rate in the GC is responsible for the high gas temperatures (e.g. Yusef-Zadeh et al. 2013). Limited observations of SiO emission toward G0.253+0.016 show an overall correlation between the $\mathrm{SiO}$ emission and high column density gas (Lis et al. 2001), however, fully-sampled maps are needed to understand this apparent correspondence and to determine the details of the gas kinematics.

To provide a more detailed picture of the dense and shocked gas within G0.253+0.016 we utilise molecular line data obtained recently as part of the Millimetre Astronomy Legacy Team $90 \mathrm{GHz}$ (MALT90) survey. This survey covers many important spectral lines within the $3 \mathrm{~mm}(90 \mathrm{GHz})$ regime. We complement these data with additional, higher frequency APEX observations (at 230 and $345 \mathrm{GHz}$ ). When combined, these data can be used to measure the global physical properties and kinematics of the gas within G0.253+0.016 and to determine whether it may be in the early stages of forming a high-mass cluster triggered by a recent close passage to Sgr A*, as suggested recently by Longmore et al. (2013b).

\section{The Millimetre Astronomy Legacy Team $90 \mathrm{GHz}$ (MALT90) survey}

G0.253+0.016 was observed in July 2010 during the first season of the MALT90 ${ }^{3}$ survey (Foster et al. 2011; Jackson et al. 2013, Rathborne et al. in prep). By observing thousands of high-mass star-forming clumps, MALT90 aims to characterise their global chemical and physical evolution.

\subsection{Survey strategy}

MALT90 utilizes the Mopra 22m telescope located near Coonabarabran in New South Wales, Australia to obtain small maps around clumps identified in the recent $870 \mu \mathrm{m}$ continuum APEX (Atacama Pathfinder Experiment) Telescope Large Area Survey of the Galaxy (ATLASGAL; Schuller et al. 2009; Contreras et al. 2013). For all maps, the 'zoom mode'

\footnotetext{
${ }^{3}$ http://malt90.bu.edu
} 
of the MOPS spectrometer was configured to a central frequency of $89.690 \mathrm{GHz}$. The zoom mode allows 16 individual IFs to be tuned to any molecular transition within a range of 8 $\mathrm{GHz}$, allowing us to simultaneously map their distribution. Each zoom band was $137.5 \mathrm{MHz}$ wide, with 4096 spectral channels, corresponding to a velocity resolution of $0.11 \mathrm{~km} \mathrm{~s}^{-1}$. At $90 \mathrm{GHz}$, Mopra's beam is $\sim 38^{\prime \prime}$ and the typical pointing accuracy is better than 8 " Foster et al. 2011).

MALT90 has obtained $3^{\prime} \times 3^{\prime}$ maps around $\sim 2,000$ ATLASGAL clumps. The maps were obtained using the On-The-Fly (OTF) mode, scanning in Galactic coordinates. To facilitate the removal of any striping artefacts or baseline variations in the maps that result from weather fluctuations, two maps were obtained by scanning in orthogonal directions: one scanning in Galactic Latitude, the other in Galactic Longitude. At the end of each scanning row a reference position was observed. An individual absolute reference position was used, located at a position a degree away from the source position in Galactic Latitude. The pointing was checked using a nearby, bright SiO maser immediately before and after the maps were obtained. The system temperature was measured via a paddle every 15 mins (two per map). Each map took $\sim 31$ mins to complete. The data reduction was performed using an automated python-based script (ASAP, Livedata and Gridzilla; see Jackson et al. 2013 for more details). The processed data cubes were downloaded from the MALT90 on-line archive 4 . The typical $\mathrm{rms}$ noise of $\mathrm{T}_{A}^{*}$ is $\sim 0.25 \mathrm{~K}$ per channel. To convert to main-beam brightness temperature $\left(\mathrm{T}_{m b}\right)$, we divide the antenna temperature $\left(\mathrm{T}_{A}^{*}\right)$ by the main-beam efficiency $\left(\eta_{m b}\right)$ of 0.49 (Ladd et al. 2005). To cover the full extent of G0.253+0.016 we combined two adjacent MALT90 maps. In all images the $(0,0)$ offset in Galactic Longitude and Latitude corresponds to the peak in the $870 \mu \mathrm{m}$ dust continuum emission at $l=0.257^{\circ}$, $b=0.016^{\circ}$.

\subsection{Observed molecular transitions}

The 16 available IFs for the MALT90 survey were tuned to the specific molecular transitions listed in Table 1 (Jackson et al. 2013). These molecules and transitions were selected because they span a range in excitation energies and critical densities, and, thus, can be used to reveal the physical and chemical state of the gas. The four brightest transitions that are typically detected towards clumps within the survey are $\mathrm{N}_{2} \mathrm{H}^{+}, \mathrm{HNC}, \mathrm{HCO}^{+}$, and $\mathrm{HCN}(1-$ $0)$. These are ground-state rotational transitions of molecules with similar critical densities and are typically detected in dense star-forming regions independent of the star formation

\footnotetext{
${ }^{4}$ http://atoa.atnf.csiro.au/MALT90
} 
activity and, thus, internal temperatures. Because $\mathrm{N}_{2} \mathrm{H}^{+}$better resists depletion at low temperatures and high-densities compared to carbon-bearing species, it traces both hot and cold gas. As such, it is a useful probe of the gas associated with all phases in the evolutionary sequence of a high-mass star-forming clump. $\mathrm{HCO}^{+}$is a good tracer of kinematics, as it often shows broad line wings indicative of outflows or line asymmetries indicative of infall. However, because these ground-state transitions are often optically thick, also included within the survey are four optically thin isotopologues: ${ }^{13} \mathrm{CS}(2-1),{ }^{13} \mathrm{C}^{34} \mathrm{~S}(2-1), \mathrm{HN}^{13} \mathrm{C}(1-0)$, and $\mathrm{H}^{13} \mathrm{CO}^{+}(1-0)$.

Several molecular transitions that trace more complex chemistry are also included within the survey: $\mathrm{CH}_{3} \mathrm{CN} 5(1)-4(1), \mathrm{HC}_{3} \mathrm{~N}(10-9), \mathrm{HC}^{13} \mathrm{CCN}(10-9)$, $\mathrm{HNCO} 4(0,4)-3(0,3)$, and HNCO 4(1,3)-3(1,2). These high excitation energy, complex carbon-bearing species trace heated gas and are often associated with 'hot molecular cores' (HMCs): regions of molecular material that are compact $(<0.1 \mathrm{pc})$, dense $\left(10^{5}-10^{8} \mathrm{~cm}^{-3}\right)$, with high temperatures $\left(\mathrm{T}_{\text {gas }}>\right.$ 100 K; Garay \& Lizano 1999; Kurtz et al. 2000; Churchwell 2002). Because their environment is heated by the UV radiation from a high-mass star, HMCs trace the immediate vicinity around a recently formed high-mass star and are an excellent signpost to the earliest phases in the formation of a high-mass star.

Also included is the $\mathrm{H} 41 \alpha$ recombination line which traces ionized gas; $\mathrm{SiO}(2-1)$ which traces shocked gas and outflows; and $\mathrm{C}_{2} \mathrm{H}(1-0) 3 / 2-1 / 2$ which, in addition to tracing cold dense gas (Vasyunina et al. 2011), can also be produced in photodissociation regions (PDRs; Lo et al. 2009; Gerin et al. 2011) when the UV radiation from a high-mass star heats and dissociates molecules on the edge of dense molecular material.

Of the molecules that have been observed, $\mathrm{HNC}, \mathrm{N}_{2} \mathrm{H}^{+}, \mathrm{C}_{2} \mathrm{H}, \mathrm{HCN}$, and $\mathrm{CH}_{3} \mathrm{CN}$ all show hyperfine splitting (listed in approximate order of increasing separation between the hyperfine components). Because the measured line-widths toward G0.253+0.016 are broad it is often difficult to separate the hyperfine components as their offset in their hyperfine splitting is significantly less than the line-width.

\subsection{Complementary APEX observations: higher J transitions}

While the molecular transitions observed as part of the MALT90 survey can reveal the location, kinematics, and opacity of the dense and shocked/heated gas, by themselves, they are insufficient to determine the physical conditions reliably. Only with complementary observations of the higher J transitions of the same molecular species can we perform a multilevel excitation analysis which provides information on the physical properties. To obtain 
these complementary data we utilised the APEX telescope, located in Llano de Chajnantor, Chile, to map emission from the $\mathrm{HNC}, \mathrm{HCN} \mathrm{HCO}^{+}$, and $\mathrm{N}_{2} \mathrm{H}^{+}(3-2)$ and (4-3), and $\mathrm{SiO}(5-$ 4) transitions.

Using the OTF mapping mode, we obtained half-beam sampled $3^{\prime} \times 3^{\prime}$ maps across G0.253+0.016 in each of the transitions listed above. The observations were conducted in September 2012. In all cases the maps were centred on the peak in the dust continuum emission and, thus, only cover the brightest part of the clump (i.e. the coverage is similar to that of the individual map from the MALT90 survey in the lower right of the moment maps in Fig. 2). We used the APEX-1 $\left(\sim 230 \mathrm{GHz}\right.$, beam size of $\left.\sim 27^{\prime \prime}\right)$ and APEX-2 $(\sim 350 \mathrm{GHz}$, beam size of $\sim 18^{\prime \prime}$ ) heterodyne receivers tuned to the transitions listed above which resulted in a velocity resolution of $\sim 1.2 \mathrm{~km} \mathrm{~s}^{-1}$. The maps took between $1-2.5$ hours to complete, depending on the observing frequency. A fixed off-source position was used for all maps. A nearby $\mathrm{SiO}$ maser was used to check the pointing accuracy approximately every hour and was typically better than $4^{\prime \prime}$. During our observations the water vapour column density ranged from $0.6-1.2 \mathrm{~mm}$ and the system temperatures were typically $200-300 \mathrm{~K}$. The data were baseline subtracted before being combined and gridded into data cubes within the GILDAS package. While each cube was obtained so that it was Nyquist sampled at the corresponding frequency, all cubes were smoothed and re-gridded in $(l, b)$ to match the beam and pixel size of the MALT90 maps. The final maps have a $\mathrm{T}_{A}^{*} 1 \sigma \mathrm{rms}$ noise sensitivity of $\sim 0.2 \mathrm{~K} \mathrm{channel}^{-1}$ in the $38^{\prime \prime}$ beam. To convert from $\mathrm{T}_{A}^{*}$ to $\mathrm{T}_{m b}$ we used a beam efficiency, $\eta_{m b}$, of 0.73 and 0.75 for the 230 and $350 \mathrm{GHz}$ data respectively (Güsten et al. 2006).

\subsection{Moment analysis, channel maps, position-velocity diagrams}

The observed emission was characterised via a moment analysis (Sault et al. 1995). This analysis is independent of Gaussian deconvolution and assumptions about individual velocity components. However, care needs to be taken in the interpretation when more than one velocity component is apparent and with molecules that show hyperfine splitting.

For each molecular transition we compute the first three moments: the zeroth moment $\left(\mathrm{M}_{0}\right.$, or integrated intensity), the first moment $\left(\mathrm{M}_{1}\right.$, the intensity weighted velocity field), and the second moment $\left(\mathrm{M}_{2}\right.$, the intensity weighted velocity dispersion, $\left.\sigma\right)$. These are defined as

$$
M_{0}=\int T_{A}^{*}(v) d v, \quad M_{1}=\frac{\int T_{A}^{*}(v) v d v}{\int T_{A}^{*}(v) d v}, \quad M_{2}=\sqrt{\frac{\int T_{A}^{*}(v)\left(v-M_{1}\right)^{2} d v}{\int T_{A}^{*}(v) d v}}
$$

where $\mathrm{T}_{A}^{*}(v)$ is the measured brightness at a given velocity $v$. The channel maps show the 
emission integrated over $\sim 6 \mathrm{~km} \mathrm{~s}^{-1}$ around the listed velocity. In all cases, the major axis of the clump is shown as the diagonal solid line on the three moment maps. The positionvelocity diagrams were made by averaging the emission over the clump's minor axis and shows the emission along the major axis (position) as a function of velocity.

\section{Results}

\subsection{Dense, hot gas: morphological match to the IR extinction}

The MALT90 data reveal extended emission toward G0.253+0.016 from 12 of the 16 transitions observed as part of the survey. In all cases, emission was also detected toward G0.253+0.016 in the higher $\mathrm{J}$ transitions observed using APEX. The four main density tracers $\left(\mathrm{N}_{2} \mathrm{H}^{+}, \mathrm{HCN}, \mathrm{HCO}^{+}\right.$, and $\mathrm{HNC} ;(1-0),(3-2)$ and (4-3)) show the brightest emission. Also detected were three isotopologues $\left(\mathrm{H}^{13} \mathrm{CO}^{+}(1-0), \mathrm{HN}^{13} \mathrm{C}(1-0)\right.$, and ${ }^{13} \mathrm{CS}(2-$ 1)) and extended emission from a number of tracers of complex chemistry and/or shocks $\left(\mathrm{CH}_{3} \mathrm{CN} 5(1)-4(1), \mathrm{C}_{2} \mathrm{H}(1-0) 3 / 2-1 / 2, \mathrm{HNCO} 4(0,4)-3(0,3), \mathrm{HC}_{3} \mathrm{~N}(10-9)\right.$, $\mathrm{SiO}(2-1)$ and $(5-4))$. Table 1 lists, for each of the detected molecular line transitions, the position of the peak (in Galactic Longitude, Galactic Latitude and LSR velocity) and the corresponding measured peak brightness temperature $\left(\mathrm{T}_{m b}\right)$. Figure 1 shows the integrated intensity image for $\mathrm{HNCO} 4(0,4)-3(0,3)$ overlaid on the mid-IR extinction and sub-mm dust continuum emission toward G0.253+0.016.

Figures 2 and 3 show the integrated intensity images for each of the detected molecular transitions. Similar to the dense gas tracers, the overall morphology of the emission from the isotopologues also match well the emission from more complex molecules that typically trace hot/shocked gas. The fact that the morphology of the emission from the dense gas tracers and complex molecules matches well the morphology of the dust continuum emission and IR extinction demonstrates that the molecular line emission originates from the clump rather than from the surrounding large-scale GC environment.

Despite the overall correspondence, there are clear differences in the location of the peaks and relative extents of the integrated emission from the various molecules and transitions. Transitions with higher critical densities and excitation temperatures are less extended compared to those with lower critical densities and excitation temperatures (i.e. compare HCN (4-3) and (1-0) in Fig. 22. Moreover, while the integrated intensities for most transitions show a single peak toward the lower part of the clump, the integrated intensities for

${ }^{13} \mathrm{CS}(2-1), \mathrm{HN}^{13} \mathrm{C}(1-0)$, and $\mathrm{HNC}(3-2)$ peak toward the clump's centre. Moreover, the integrated intensities from $\mathrm{HNCO} 4(0,4)-3(0,3), \mathrm{HC}_{3} \mathrm{~N}(10-9), \mathrm{HCO}^{+}(3-2)$, and $\mathrm{HCN}(3-2)$ 
and (4-3) show two peaks. These differences arise from variations in the excitation, optical depth, and chemistry within the clump.

\subsection{Gas density and temperature}

While the critical densities of the observed transitions are all high $\left(10^{5}-10^{7} \mathrm{~cm}^{-3}\right)$, the excitation energies of the 'hot core' molecules $\mathrm{E}_{u} / k$ of $10-20 \mathrm{~K}$ are much higher compared to $\mathrm{E}_{u} / k$ of $\sim 4 \mathrm{~K}$ for the dense gas tracers (see Table 11). The presence of the hot core molecules usually indicates the location of gas that has either a high temperature (i.e. > $100 \mathrm{~K}$ ) and/or high density (i.e. the gas density is well above the critical density, $n_{\text {crit }}$ ): these conditions are needed for both their formation and excitation. For the observed molecules, $n_{\text {crit }}$ is $\sim 10^{5}-10^{7} \mathrm{~cm}^{-3}$ (Table 1). Toward G0.253+0.016 the average volume density is $\sim 2$ $\times 10^{4} \mathrm{~cm}^{-3}$ (calculated using a radius equal to the geometric mean of the major and minor axes and that assuming the same extent along the line of sight). If the gas is not highly clumped, this volume density falls well below the critical densities for these molecules, and thus, the excitation would be sub-thermal (i.e. $\mathrm{n}<\mathrm{n}_{\text {crit }}$ ). In other words, if the gas is approximately uniformly distributed, the excitation temperatures $\left(\mathrm{T}_{e x}\right)$ will be significantly lower than the gas kinetic temperatures $\left(\mathrm{T}_{K}\right)$. However, because this volume density is averaged over parsec size-scales, the local volume density may be significantly higher if the gas is clumpy and highly structured on small scales. Thus, small regions with higher volume densities may lie at or above the critical density (i.e. $\mathrm{n} \sim \mathrm{n}_{c r i t}$ ), and in these regions $\mathrm{T}_{e x}$ will approach $\mathrm{T}_{K}$.

Using the combination of the MALT90 and APEX datasets, we can investigate the gas density and temperature within G0.253+0.016 by means of excitation and radiative transfer modelling. Figure 4 plots the expected line ratios for $\mathrm{HNC}$ as a function of volume density and kinetic temperature. This plot was generated using RADEX, a non-LTE radiative transfer model (van der Tak et al. 2007) assuming a spherical cloud and a uniform density and temperature. The input column density of $\operatorname{HNC}\left(10^{15} \mathrm{~cm}^{-2}\right)$ was estimated from the dust-derived column density measured toward G0.253+0.016 (Longmore et al. 2012) and assuming an abundance of $\mathrm{HNC}$ relative to $\mathrm{H}_{2}$ of $3 \times 10^{-9}$ (Schilke et al. 1992).

For transitions that are both optically thin and with the same excitation conditions, the line intensity ratio equals the abundance ratio of the two species (abundance ratio of ${ }^{12} \mathrm{C} /{ }^{13} \mathrm{C}$ is $\sim 20$ for the GC region; Wilson (1999); Savage et al. (2002)). If both transitions are optically thick and their emitting regions have the same excitation conditions, then the intensity ratio is unity. Using the detected isotopologues we find line intensity ratios of $\sim 4-6$ from $\mathrm{HNC}$ and $\mathrm{HN}^{13} \mathrm{C}(1-0)$ and slightly higher ratios of $\sim 6-8$ from $\mathrm{HCO}^{+}$and 
$\mathrm{H}^{13} \mathrm{CO}^{+}(1-0)$. These ratios give an estimate of the opacity of the ${ }^{12} \mathrm{C}$ molecules of $2-3$, which implies that the emission is moderately optically thick.

Overlaid on Figure 4 are the observed line ratios toward three positions within G0.253+0.016 (solid lines, P2, P3 and P4, see Fig. 6). Assuming that the beam filling factor is similar for both transitions, the HNC (4-3) to (3-2) line ratios indicate a gas density of $\sim 10^{6} \mathrm{~cm}^{-3}$. This derived density is an order of magnitude higher than that derived toward G0.253+0.016 from LVG modelling of the emission from CS and $\mathrm{C}^{34} \mathrm{~S}$ (Lis et al. 2001). The large disparity between the average volume density and the implied local volume density indicated by the line ratios indicates that the gas is highly clumped, at least in the gas traced by HNC. The models, however, should be treated with caution if multiple gas components with different temperatures and densities are present along the same line of sight, especially for optically thick lines, where radiative transfer effects can significantly alter the observed line ratios. Indeed, no single component solution exists for $\mathrm{HCO}^{+}$emission.

While the kinetic temperature cannot be well constrained by our data, recent $\mathrm{H}_{2} \mathrm{CO}$ observations that are sensitive to the gas temperature indicate a kinetic temperature of 65 ${ }_{-10}^{+20}$ or $70_{-15}^{+25}$ (depending on the assumed abundance; Ao et al. 2013). Within the errors, this kinetic temperature is indistinguishable from the global kinetic temperature of the molecular gas within the broader GC region $(65 \pm 10 \mathrm{~K}$; Ao et al. 2013).

\subsection{Comparison between the dust and gas column density distribution}

If both the dust and gas were optically thin and traced the same material throughout the clump, then the integrated intensity of an optically thin gas tracer ought to follow the column density profile derived from the dust. Instead, we find that regardless of the gas tracer, their integrated intensities peak at different locations when compared to the dust column density. Figure 5 shows the normalised dust column density profile along the major axis of G0.253+0.016 overlaid with $70 \mu \mathrm{m}$ extinction profile and the integrated intensity emission profiles from three different gas tracers $\left(\mathrm{HNC}, \mathrm{HN}^{13} \mathrm{C}\right.$, and $\mathrm{HNCO}$ ).

Overall, the dust column density profile is matched well by the $70 \mu \mathrm{m}$ extinction profile. In contrast, the line emission profiles appear anti-correlated with the dust column density toward the clump's centre, regardless of the gas tracer (i.e. $\mathrm{HNC}, \mathrm{HN}^{13} \mathrm{C}$, or $\mathrm{HNCO}$; these three molecules were selected to represent the emission from the dense gas tracers, isotopolgues, and hot gas tracers respectively).

The Herschel dust emission indicates a peak $\mathrm{H}_{2}$ column density of $3.3 \times 10^{23} \mathrm{~cm}^{-2}$ and a total mass for the clump of $1.3 \times 10^{5} \mathrm{M}_{\odot}$. The lack of molecular line emission (particularly 
from the isotopologues, e.g. $\mathrm{HN}^{13} \mathrm{C}$ ) at the peak of the dust column density is therefore surprising. Indeed, none of the molecular lines peak toward the dust column density peak. If the molecular line emission is optically thin, then the most plausible explanation is that there is severe chemical gas depletion in the clump's cold interior. Depletion arises when the gas and dust have a low temperature and high column density $\left(\mathrm{T}_{\text {gas }} \sim \mathrm{T}_{\text {dust }} \sim 10 \mathrm{~K}, \mathrm{~N}\left(\mathrm{H}_{2}\right)\right.$ $>10^{22} \mathrm{~cm}^{-2}$, e.g., Redman et al. 2002), and the molecular gas freezes onto the dust grains in the form of ice. In these regions, despite a large mass of dust, the usual molecular markers of the gas mass are absent because these molecular species are frozen and hence do not emit in molecular lines. While the dust conditions inferred for the center of G0.253+0.016 have precisely the properties required for chemical depletion, the gas temperature may be slightly higher (a few $10 \mathrm{~K}$ ). Recent modelling of the gas and dust temperature distribution within G0.253+0.016, shows that for densities of $10^{4} \mathrm{~cm}^{-3}$, the gas temperature is roughly $70 \mathrm{~K}$, while the dust temperature may be closer to $20 \mathrm{~K}$ (Clark et al. 2013). However, as the density increases (presumably toward the clump's centre) and the dust temperature decreases $(\sim 10 \mathrm{~K})$, then the gas and dust temperatures should become more strongly coupled and the gas temperature will be closer to the dust temperature. Detailed radiative transfer modelling is clearly needed to determine the exact gas temperature distribution within the clump. Nevertheless, based on these observations we suggest that G0.253+0.016 is centrally condensed with a cold, dense interior in which the gas is depleted. The lack of molecular line emission toward the clump center therefore simply reflects the absence of these molecular species in the gas phase due to depletion.

\subsection{Velocity gradient, complex gas kinematics}

Figure 6 shows, for HNCO, the three moment maps, the channel maps, and positionvelocity $(p v)$ diagram (similar figures for all other detected molecules transitions are included on-line, Figs. 10 29). If the emission arises from a single clump, then a velocity gradient is clearly evident from the intensity weighted velocity field. Included on the position-velocity diagrams is a solid diagonal line marking the HNCO velocity gradient seen across G0.253+0.016 $\left(\sim 18.6 \mathrm{~km} \mathrm{~s}^{-1} \mathrm{pc}^{-1}\right)$.

Figure 7 shows spectra at 5 positions across G0.253+0.016. The spectra were extracted at the positions marked on the integrated intensity image show in Figure 6 and were selected to show details of the kinematics across the clump. We group the molecules into three separate panels based on whether they typically trace emission that is likely to be optically thick ( $\mathrm{HCN}, \mathrm{HCO}^{+}, \mathrm{N}_{2} \mathrm{H}^{+}$, $\mathrm{HNC}$; upper panels), optically thin $\left(\mathrm{HN}^{13} \mathrm{C}, \mathrm{H}^{13} \mathrm{CO}^{+},{ }^{13} \mathrm{CS}\right.$; middle panels), or from the higher excitation energy 'hot core' molecules $\left(\mathrm{HC}_{3} \mathrm{~N}, \mathrm{HNCO}\right.$, 
$\mathrm{SiO}, \mathrm{CH}_{3} \mathrm{CN}$; lower panels). Although the line profiles within each of these groups are quite similar, the profiles differ in a systematic way between the optically thick lines and the other two optically thin and hot gas categories.

To emphasize their systematic differences, we remove the clump's velocity gradient using the velocity determined from the intensity-weighted velocity field of HNCO (we select this transition as it shows well the velocity gradient and the emission is detected with a high signal to noise). Plotting the spectra in this way simplifies their interpretation.

For each position, we plot the spectra on a velocity axis relative to the derived $\mathrm{V}_{L S R}$ at that position. The derived $V_{L S R}$ for each position is shown at the top of each column of Figure 7 and is marked by the solid vertical line in each spectrum at the velocity offset of $0 \mathrm{~km} \mathrm{~s}^{-1}$. The vertical dotted lines delineate the velocity range we attribute to the emission from G0.253+0.016 $\left( \pm 35 \mathrm{~km} \mathrm{~s}^{-1}\right.$ around the derived $\left.\mathrm{V}_{L S R}\right)$. Evident in the $\mathrm{HCO}^{+}\left(1^{-}\right.$ 0 ) spectra are two other molecular clouds along the line of sight (at $V_{L S R}$ of -40 and $\left.75 \mathrm{~km} \mathrm{~s}^{-1}\right)$. These are unrelated clouds 5 and as such we exclude their emission from all subsequent analyses.

Toward all selected positions, the spectra from the optically thin tracers and those from the hot/shocked gas have similar profiles: a single velocity component is seen toward the edge of the cloud (P1, P4 and P5), while toward its centre (P2 and P3) there are two apparent velocity components. In contrast, spectra from the optically thick gas tracers show that toward all positions the emission peaks red-ward of the other species and of the derived $\mathrm{V}_{L S R}$. The consistent pattern of optically thick lines peaking at more positive velocities than the optically thin or hot core lines persists regardless of the local systemic velocity of the cloud. In other words, the redshift of the optically thick lines with respect to the optically thin and hot core lines is independent of the overall velocity gradient of the cloud.

The $\mathrm{HCO}^{+}$and $\mathrm{HCN}$ profiles toward P1 and P2 show additional blue-shifted absorption at $\mathrm{V}_{L S R}$ near $0 \mathrm{~km} \mathrm{~s}^{-1}$. Because all gas in circular Galactic orbits has a radial velocity of $0 \mathrm{~km} \mathrm{~s}^{-1}$ toward the Galactic Center, any cold gas in the intervening $8.5 \mathrm{kpc}$ will absorb at this velocity. Indeed, a $0 \mathrm{~km} \mathrm{~s}^{-1}$ absorption feature is widely seen throughout the CMZ and is most obvious from the molecules that are widespread, with low excitation energies (e.g.

\footnotetext{
${ }^{5}$ While the $75 \mathrm{~km} \mathrm{~s}^{-1}$ cloud is clearly detected in $\mathrm{HCO}^{+}(1-0)$ and $\mathrm{SiO}(2-1)$, we believe it is unrelated to G0.253+0.016. In the 'elliptical ring' model presented recently by Molinari et al. (2011) based on molecular line velocities, it is thought that the $75 \mathrm{~km} \mathrm{~s}^{-1}$ cloud lies on the back side of the ring, compared to G0.253+0.016 which lies on the front side. Moreover, the location of these two clouds lies close to the "cross over' point of the ring, where the front and back sides cross our line of sight. The fact that G0.253+0.016 is IR-dark while the $75 \mathrm{~km} \mathrm{~s}^{-1}$ cloud is not, also argues that they are unrelated and probably at different physical locations along the line of sight.
} 
$\mathrm{HCO}^{+}$and $\mathrm{HCN}$; Jones et al. 2012).

Figure 8 compares the emission profiles from several different transitions of the same molecular species. We select $\mathrm{HCO}^{+}$as we have observed it in four different transitions and it does not have hyperfine components. Because the molecular transitions are tracers of material at different critical densities and excitation energies, their relative extent, location and velocity profiles can be used to trace density and temperature gradients within the cloud. The emission from the different transitions peak at different velocities: transitions with higher critical densities peak closer to the local systemic velocity derived from the optically thin lines.

\subsection{Small-scale structure}

Given an effective radius of $2.9 \mathrm{pc}$ and a dust-derived mass of $\sim 1.3 \times 10^{5} \mathrm{M}_{\odot}$, the average $\mathrm{H}_{2}$ volume density of G0.253+0.016 is $\sim 2 \times 10^{4} \mathrm{~cm}^{-3}$. For this density, the estimated free-fall time is $\sim 0.2$ Myrs. Because this is comparable to the turbulent crossing time of $\sim$ 0.4 Myrs, the clump should be undergoing gravitational collapse and fragmentation. Indeed, the $450 \mu \mathrm{m}$ dust continuum emission shows substructure down to the limit of the angular resolution of the data $\left(7.5^{\prime \prime} ; 0.3 \mathrm{pc}\right.$; Longmore et al. 2012).

With a kinetic temperature of $\sim 70 \mathrm{~K}$ and a volume density of $\sim 10^{6} \mathrm{~cm}^{-3}$ RADEX models can be used to generate the expected line intensities. The input column density of HNC $\left(10^{15} \mathrm{~cm}^{-2}\right)$ was estimated from the dust-derived column density measured toward G0.253+0.016 and assuming an abundance of $\mathrm{HNC}$ relative to $\mathrm{H}_{2}$ of $3 \times 10^{-9}$ (Schilke et al. 1992). For this temperature and density, the predicted HNC (1-0), (3-2), and (4-3) line brightness temperatures are $\sim 48, \sim 34$, and $\sim 27 \mathrm{~K}$ respectively for homogeneous gas that fills the beam. These intensities are much higher than what is measured toward G0.253+0.016, which suggests that the gas could be highly clumped on small scales and that the smaller observed intensities result from low beam-filling factors. Indeed, the channel maps clearly show that the emission has structure in both position and velocity (e.g. Fig. 6). While the angular resolution of our current data is insufficient to pinpoint the individual small-scale fragments, the data are consistent with a clump that is highly sub-structured.

The ratio of the predicted to measured line intensities can be used to estimate the beam filling factor. We find values of $\sim 0.032, \sim 0.025$, and $\sim 0.019$ for the HNC $(1-0),(3-2)$, and (4-3) lines respectively. The general trend of a higher filling factor for the lower J transitions is expected as the emission from the lower $\mathrm{J}$ transitions traces less dense, colder material that is presumably more widespread within the beam. 
The inferred clumping of the molecular emission, however, depends on the validity of the RADEX model assumption of uniform gas properties throughout the cloud. If multiple components of different temperatures and densities exist along the line of sight, then the model results may be invalid because the radiative transfer and optical depth effects are not properly accounted for. For example, if the optically thick (1-0) lines are suppressed through self-absorption, RADEX would overpredict the densities and brightness temperatures due to artificially high $(3-2) /(1-0)$ line ratios.

Higher angular resolution observations are clearly needed to determine the small-scale structure of G0.253+0.016. Despite their significant improvement in angular resolution, recent CARMA and SMA observations of this clump show a lack of dense gas on spatial scales of $\sim 2^{\prime \prime}$, which led Kauffmann et al. (2013) to speculate that the clump has insufficient dense material to form stars. This result is puzzling if the gas in fact is highly clumped with high brightness temperatures as the RADEX modelling suggests. Our recent ALMA cycle 0 observations of the $90 \mathrm{GHz}$ line and continuum emission toward G0.253+0.016 show many compact fragments in dense gas tracers (1.7"; Rathborne et al. 2013). The latter detection of the small-scale dense gas is a result of the improvement in sensitivity provided by ALMA and will be discussed in future work.

\section{Discussion}

The presence of cold dust, 'hot core' chemistry, and complicated kinematics within G0.253+0.016 is intriguing and may provide clues as to how this clump formed and whether it is on the verge of collapse. While the presence of cold dust, complex chemistry, and broad line-widths is well documented in the CMZ (e.g., Wilson et al. 1982; Martín-Pintado et al. 2001), G0.253+0.016 is extreme compared to more typical clumps in the Galactic disk as it has a high-density, shows little star formation, and has sufficient mass to form a YMC through direct collapse.

In this section we discuss the formation of G0.253+0.016 and posit two scenarios that may explain the presence of the hot gas within G0.253+0.016 with very different predictions for its distribution and kinematics.

\subsection{The formation of G0.253+0.016 via a close passage to Sgr A*}

Longmore et al. (2013b) suggest that the formation of G0.253+0.016 has been triggered by the pericentre passage of a gas stream close to the bottom of the Galactic gravitational 
potential near Sgr A*, during which the gas is stretched in the orbital direction, but compressed in the direction perpendicular to its orbit. It is then argued that this compression leads to an accelerated dissipation of turbulent energy and hence triggers star formation. In this picture, G0.253+0.016 recently passed pericentre $\sim 0.6$ Myrs ago and therefore should be on the verge of initiating star formation, whereas clouds like Sgr B2 that passed pericentre 1-2 Myr ago should be exhibiting prevalent star formation. This scenario accounts for many of the observed properties of Galactic Center clouds.

If this scenario accurately describes G0.253+0.016, then its molecular line emission ought to show: (1) that the dynamical time scales of its motions are comparable to the time since pericentre passage ( 0.6 Myr in the model of Molinari et al. 2011), (2) that it is elongated in the direction of its orbital motion, and (3) that there is evidence for bulk radial motions as the cloud is stretched and compressed.

Each of these predictions are consistent with the observed properties of G0.253+0.016. Firstly, the implied dynamical time scale for radial motions is $\sim 0.6 \mathrm{Myr}$, in good agreement with the estimated time since closest passage to the Galactic Center in the Molinari et al. (2011) model (the optically thick lines peak at velocities red-ward of the optically thin lines by $\sim 5 \mathrm{~km} \mathrm{~s}^{-1}$ and its effective radius is $2.9 \mathrm{pc}$, leading to a dynamical time scale, $t=r / v$, of $0.6 \mathrm{Myr}$ ). Second, the morphology of G0.253+0.016 is indeed elongated, in the predicted direction along its orbit. Third, the observations clearly show bulk radial motions, as evidenced by the systematic redshift of the optically thick emission with respect to the optically thin and hot gas tracers.

In the scenario where G0.253+0.016 results from a pericentre passage, preliminary results from numerical simulations of this process (Kruijssen et al. in preparation) show that its center (within the tidal radius) will dissipate its turbulent energy at an accelerated rate and will therefore collapse, while its diffuse outer envelope (outside the tidal radius) will be stripped. Because both the collapsing center and the stripped envelope are characterised by radial motions, it is not clear whether the observed optically thick molecular lines are probing the collapsing center or the expanding envelope. Nevertheless, in either case, radial motions are clearly seen in the molecular line data presented here. The exact comparison to the numerical simulations will be presented in a future paper.

\subsection{Two clumps colliding}

One scenario for the presence of hot gas within G0.253+0.016 is that it has formed recently as a result of clump-clump collisions. In this scenario, the shocks from the collisions 
are responsible for heating the gas. Indeed, widespread shocks, traced via $\mathrm{SiO}$, are clearly associated with G0.253+0.016 (Lis et al. 2001; Kauffmann et al. 2013). From large-scale CO mapping toward G0.253+0.016, Lis \& Menten (1998) found evidence for a velocity gradient and complex kinematics within the clump that they interpret to be the superposition of two spatially overlapping components that may be interacting. In this collision/interaction scenario, the dust is not thermally coupled to the gas and, thus, has not yet had time to be heated. If true, then we would expect to see two velocity components in the dense gas tracers and a central zone of hot gas at the site of the collision. The hot/shocked gas should coincide with the position and velocity of the collision site.

While our observations show two velocity components in the dense gas tracers toward the centre of the clump where presumably the components are interacting, the hot/shocked gas tracers are not isolated to this central region ( in position or velocity). Instead, the hot/shocked gas has similar distribution and kinematics to the optically thin gas tracers and, thus, arises from similar regions across the clump. The fact that the emission from the hot/shocked gas is spread over the whole clump argues against this simple clump-clump collision scenario as a mechanism for producing the hot/shocked gas. There is no evidence for a distinct interaction zone.

\subsection{A centrally condensed clump, with depletion in its cold interior}

An alternative scenario is one in which the clump is a single, coherent structure, with the hot gas distributed throughout. When comparing the emission from different transitions of the same molecule, the integrated intensity images and position-velocity diagrams show that the (1-0) emission is more extended compared to the emission from the (3-2) and (43) transitions and isotopologues (for $\mathrm{HCO}^{+}$see Figs. 20, 21, 22 and 23). Moreover, their spectra (Fig. 8) show that the emission from the different transitions also peak at different velocities. Our data are consistent with a gradient in velocity that follows the critical density of the transitions: emission from the higher J transitions peak toward a more central velocity. This velocity shift combined with a decrease in the size of the emitting region in the higher density gas indicates a density gradient of material that is centrally condensed.

Moreover, the observed anti-correlation between the dust column density and the molecular integrated intensity toward the clump's centre shows that the molecules are absent in the highest density region at the clump's center. One possible explanation for this disparity is molecular depletion in its cold interior. Thus, the absence of emission from the optically thin species toward the clump's center and systemic velocity may simply reflect depletion in the cloud's cold, dense interior. If true, then the two velocity components observed in 
the both the optically thin and hot/shocked gas are not tracing physically distinct clumps, but are instead simply tracing the velocity fields of a centrally condensed clump. In this scenario, the apparent presence of two distinct velocity components arises from the lack of emission in the center of a large, extended clump with a smooth velocity gradient.

\subsection{Radial motions}

The fact that the observed velocity field of the heated/shocked gas matches well the optically thin isotopologues implies that the emission from all of these molecules are tracing the same material. In contrast, we find that the optically thick gas is always red-shifted with respect to the optically thin/hot gas tracers (e.g. Fig. 7). Because the optically thick lines probe the $\tau=1$ surface, their persistent redshift with respect to the optically thin and hot gas tracers demonstrates that the cloud has radial motions and that the gas properties vary along the line of sight. Such asymmetries arise from the fact that the radial motions separate these distinct gas components since they have different radial velocities. Such an effect cannot arise from a foreground cloud; because of the large velocity gradient, such a foreground cloud would have to have exactly the same velocity gradient to absorb at precisely the right velocity to maintain the constant red-ward asymmetry.

\subsubsection{P Cygni profile: an expanding, centrally condensed clump}

One standard interpretation of this red-ward asymmetry is a 'P Cygni' type line profile due to expanding motions (see Fig. 9 for a schematic). In this interpretation, the blue-shifted material arises from the outer surface of the cloud, and the red-shifted material from the cloud's interior. The brighter emission at red-shifted velocities then arises from the fact that the interior of the cloud has a significantly higher excitation temperature than the exterior portion $\left(\mathrm{T}_{e x}\right.$ inner $>\mathrm{T}_{e x}$ outer). The lower excitation temperature in the exterior layers could be due to colder temperatures in the exterior if the gas is thermalized $\left(\mathrm{n}>>\mathrm{n}_{\text {crit }}\right)$, or to sub-thermal excitation $\left(\mathrm{n}<<\mathrm{n}_{\text {crit }}\right)$. The lower excitation exterior will not only be fainter than the higher excitation temperature interior, it can also absorb line emission from the

interior. Combined, these effects lead to substantial red-blue asymmetries in the line profiles of optically thick gas. 


\subsubsection{Baked Alaska: a collapsing, centrally condensed clump}

An alternative to this 'P Cygni' interpretation is a 'Baked Alaska' collapse model where the clump is externally heated $\left(\mathrm{T}_{K}\right.$ outer $>\mathrm{T}_{K}$ inner $)$. For a collapsing cloud, redshifted optically thick emission arises from the cloud's exterior and blue-shifted emission from the cloud's interior. If G0.253+0.016 is indeed collapsing, the brighter redshifted emission arises from the fact that the exterior layers have a higher excitation temperature than the cloud's interior (see Fig. 9 for a schematic). In this scenario, the gas is thermalized throughout the cloud $\left(\mathrm{n}>>\mathrm{n}_{\text {crit }}\right)$. This hot-exterior, cold-interior 'Baked Alaska' model is consistent with the recent observations and $\mathrm{SPH}$ modelling of the dust and gas temperature distribution in G0.253+0.016: both indicate that the clump is externally heated (Lis et al. 2001; Longmore et al. 2012; Clark et al. 2013). Thus, there is some evidence to support the idea that the radial motions indicate collapse, a scenario which also accounts nicely for the extremely high mass and density of the cloud.

\subsection{The cluster formation potential of G0.253+0.016 and the implications for the formation of high-mass, bound clusters}

The fact that G0.253+0.016 may be unique in the Galaxy in terms of its high density, high mass, and lack of prevalent star formation may be important for understanding how high-mass, bound clusters are formed. While the simplest idea for the formation of a highmass cluster is through direct collapse of a large, high-mass, dense and cold molecular clump progenitor, there may in fact be multiple channels for the formation of high-mass star clusters. Rather than the simple collapse of a single molecular clump, high-mass clusters may form more slowly via gradual star formation as gas is continually accreted onto a central potential well from a more extend reservoir of material. While plausible, the observed lack of age spreads in young high-mass clusters argues against this formation scenario (Clark et al. 2005; Negueruela et al. 2010; Kudryavtseva et al. 2012). Massive clusters may also form through mergers of smaller groups of stars that have already formed but are part of a common potential well (e.g., McMillan et al. 2007; Allison et al. 2009).

The observed gas morphology and kinematics suggest that G0.253+0.016 is indeed a single, coherent high-mass dense, clump that may be highly fragmented on small spatial scales. As such, it has the potential to form a high-mass cluster. The puzzle, however, is how a dense, $10^{5} \mathrm{M}_{\odot}$ clump forms without rapidly producing stars. For G0.253+0.016, its location in the CMZ may provide the clue: the increased turbulence in the CMZ may in fact inhibit star formation below a column density threshold which is much higher in the CMZ than in the Galactic plane (Kruijssen et al. 2013). However, the fact that some of the most 
massive Galactic YMCs are located outside of the Galactic centre region (e.g., Westerlund 1, Glimpse-C01, NGC 3603, RSGCs) suggests that the unique conditions within the CMZ are not essential for their formation.

High-mass clusters are thought to form quickly, perhaps in less than a few Myrs. Recent observations of NGC 3603 and Westerlund 1 suggests that the age spread within the stellar population may be <0.4 Myr (Clark et al. 2005; Negueruela et al. 2010; Kudryavtseva et al. 2012). The fact that G0.253+0.016 is one of a handful of clumps with sufficient mass to form a YMC may support this fast formation scenario; at any given time the number of

high-mass cluster precursors within the Galaxy will be limited to just a few (Longmore et al. 2012). If true, then perhaps we are catching G0.253+0.016 at a very special time, immediately before the formation of the high-mass cluster. In this case, we would expect the gas and dust to be highly fragmented, the fragments being the precursors to the individual stars or sub-clusters. Recent models do predict that G0.253+0.016 should form a bound cluster through hierarchical fragmentation (Kruijssen 2012). While our data show evidence for fragmentation, higher-angular resolution data of both the optically thin and shocked gas tracers down to small scales $(<0.1 \mathrm{pc})$ are clearly needed to definitively determine whether or not $\mathrm{G} 0.253+0.016$ with give rise to a cluster in the future.

\section{Summary}

Utilizing molecular line data from the MALT90 survey combined with complementary APEX observations, we have investigated the global conditions and kinematics of the gas within G0.253+0.016. These data reveal a wealth of information because they combine molecular transitions that cover a broad range in critical densities and excitation energies and also include a number of key transitions that trace regions of complex chemistry and shocked gas.

While G0.253+0.016 appears dark in the mid- to far-IR, implying both a low dust temperature and high column density, the presence of widespread emission from tracers of hot gas suggests that its gas temperature may be significantly higher than its measured dust temperature, consistent with previous observations. The data show that the gas has substructure and complex kinematics. Both the observed broad line-widths and the presence of shocks and complex molecular line emission from within G0.253+0.016 is consistent with its location in the CMZ. The observed morphology and kinematics of the molecular line emission are tracing gas within a single, centrally condensed clump. The absence of gas emission toward the large dust column density peak at the clump's centre probably results from gas depletion in its cold interior. The systematic red-shift of the optically thick transitions com- 
pared with the optically thin and hot gas tracers demonstrate that G0.253+0.016 exhibits radial motions. If expanding, the outer portions have lower excitation temperatures than the inner portions. If contracting, the outer portions have higher excitation temperatures than the inner portions. Because the dust temperatures do in fact indicate external heating, the collapse model is consistent with the observations. No matter which interpretation is correct, the fact that the optically thick lines all peak at redder velocities than the optically thin and hot core lines independent of the local systemic velocity clearly demonstrates systematic radial motions in $\mathrm{G} 0.253+0.016$.

With such a high density G0.253+0.016 should be undergoing gravitational collapse and fragmentation, however, its rapid star formation may have been delayed due to the high turbulence and increased gas temperatures in the CMZ. Single component excitation models suggest, from the measured line ratios, that the gas appears to be clumped: higher angular resolution data is clearly needed to pinpoint these individual regions.

Because G0.253+0.016 is an excellent candidate for the progenitor of a high-mass cluster, reliably determining the distribution, size, mass, and motion of its small-scale fragments will be critical to reveal important clues about the resulting stellar cluster and how such a cluster is formed. Future ALMA observations will reveal these properties: only with this significant increase in sensitivity, angular resolution, and dynamic range can we begin to measure the initial conditions within protoclusters and test cluster formation models.

We thank the referee for their detailed comments which have improved the clarity of the paper considerably. We are grateful to both Peter Schilke and Thomas Moeller for assistance and advice using the RADEX radiative transfer modelling code. This publication is based on data acquired with the Mopra radio telescope and the Atacama Pathfinder Experiment (APEX). The Mopra radio telescope is part of the Australia Telescope National Facility which is funded by the Commonwealth of Australia for operation as a National Facility managed by CSIRO. The University of New South Wales Digital Filter Bank used for the observations with the Mopra Telescope was provided with support from the Australian Research Council. APEX is a collaboration between the Max-Planck-Institut fur Radioastronomie, the European Southern Observatory, and the Onsala Space Observatory

Facilities: Mopra, APEX.

\section{REFERENCES}

Allison, R. J., Goodwin, S. P., Parker, R. J., de Grijs, R., Portegies Zwart, S. F., \& Kouwen- 
hoven, M. B. N. 2009, ApJ, 700, L99

Ao, Y., Henkel, C., Menten, K. M., Requena-Torres, M. A., Stanke, T., Mauersberger, R., Aalto, S., Mühle, S., \& Mangum, J. 2013, A\&A, 550, A135

Bania, T. M., Stark, A. A., \& Heiligman, G. M. 1986, ApJ, 307, 350

Bastian, N. 2008, MNRAS, 390, 759

Breen, S. L. \& Ellingsen, S. P. 2011, MNRAS, 416, 178

Churchwell, E. 2002, ARA\&A, 40, 27

Clark, J. S., Negueruela, I., Crowther, P. A., \& Goodwin, S. P. 2005, A\&A, 434, 949

Clark, P. C., Glover, S. C. O., Ragan, S. E., Shetty, R., \& Klessen, R. S. 2013, ApJ, 768, L34

Contreras, Y., Schuller, F., Urquhart, J. S., Csengeri, T., Wyrowski, F., Beuther, H., Bontemps, S., Bronfman, L., Henning, T., Menten, K. M., Schilke, P., Walmsley, C. M., Wienen, M., Tackenberg, J., \& Linz, H. 2013, A\&A, 549, A45

Davies, B., Bastian, N., Gieles, M., Seth, A. C., Mengel, S., \& Konstantopoulos, I. S. 2011, MNRAS, 411, 1386

Elmegreen, B. G. \& Efremov, Y. N. 1997, ApJ, 480, 235

Figer, D. F., Kim, S. S., Morris, M., Serabyn, E., Rich, R. M., \& McLean, I. S. 1999, ApJ, 525,750

Figer, D. F., MacKenty, J. W., Robberto, M., Smith, K., Najarro, F., Kudritzki, R. P., \& Herrero, A. 2006, ApJ, 643, 1166

Foster, J. B., Jackson, J. M., Barnes, P. J., Barris, E., Brooks, K., Cunningham, M., Finn, S. C., Fuller, G. A., Longmore, S. N., Mascoop, J. L., Peretto, N., Rathborne, J., Sanhueza, P., Schuller, F., \& Wyrowski, F. 2011, ApJS, 197, 25

Garay, G. \& Lizano, S. 1999, PASP, 111, 1049

Gerin, M., Kaźmierczak, M., Jastrzebska, M., Falgarone, E., Hily-Blant, P., Godard, B., \& de Luca, M. 2011, A\&A, 525, A116

Ginsburg, A., Bressert, E., Bally, J., \& Battersby, C. 2012, ApJ, 758, L29

Guesten, R., Walmsley, C. M., \& Pauls, T. 1981, A\&A, 103, 197 
Güsten, R., Nyman, L. Å., Schilke, P., Menten, K., Cesarsky, C., \& Booth, R. 2006, A\&A, 454, L13

Immer, K., Menten, K. M., Schuller, F., \& Lis, D. C. 2012, A\&A, 548, A120

Jackson, J. M., Rathborne, J. M., Foster, J. B., Whitaker, J. S., Sanhueza, P., Claysmith, C., Mascoop, J. L., Wienen, M., Breen, S. L., Herpin, F., Duarte-Cabral, A., Csengeri, T., Longmore, S., Contreras, Y., Indermuehle, B., Barnes, P. J., Walsh, A. J., Cunningham, M. R., Brooks, K. J., Britton, T. R., Voronkov, M. A., Urquhart, J. S., Alves, J., Jordan, C. H., Hill, T., Hoq, S., Finn, S., C., S., Bains, I., Bontemps, S., Bronfman, L., Caswell, J. L., Deharveng, L., Ellingsen, S. P., Fuller, G. A., Garay, G., Green, J. A., Hindson, L., Jones, P. A., Lenfestey, C., Lo, N., Lowe, V., Mardones, D., Menten, K. M., Minier, V., Morgan, L. K., Motte, F., Muller, E., Peretto, N., Purcell, C. R., Schilke, P., Schneider-Bontemps, N., Schuller, F., Titmarsh, A., Wyrowski, F., \& Zavagno, A. 2013, ArXiv e-prints

Jones, P. A., Burton, M. G., Cunningham, M. R., Requena-Torres, M. A., Menten, K. M., Schilke, P., Belloche, A., Leurini, S., Martín-Pintado, J., Ott, J., \& Walsh, A. J. 2012, MNRAS, 419, 2961

Kauffmann, J., Pillai, T., \& Zhang, Q. 2013, ApJ, 765, L35

Kruijssen, J. M. D. 2012, MNRAS, 426, 3008

Kruijssen, J. M. D. \& Cooper, A. P. 2012, MNRAS, 420, 340

Kruijssen, J. M. D., Longmore, S. N., Elmegreen, B. G., Murray, N., Bally, J., Testi, L., \& Kennicutt, Jr., R. C. 2013, MNRAS submitted, arXiv:1303.6286

Kudryavtseva, N., Brandner, W., Gennaro, M., Rochau, B., Stolte, A., Andersen, M., Da Rio, N., Henning, T., Tognelli, E., Hogg, D., Clark, S., \& Waters, R. 2012, ApJ, 750, L44

Kurtz, S., Cesaroni, R., Churchwell, E., Hofner, P., \& Walmsley, C. M. 2000, Protostars and Planets IV, 299

Ladd, N., Purcell, C., Wong, T., \& Robertson, S. 2005, PASA, 22, 62

Lis, D. C. \& Carlstrom, J. E. 1994, ApJ, 424, 189

Lis, D. C. \& Menten, K. M. 1998, ApJ, 507, 794

Lis, D. C., Serabyn, E., Zylka, R., \& Li, Y. 2001, ApJ, 550, 761 
Lo, N., Cunningham, M. R., Jones, P. A., Bains, I., Burton, M. G., Wong, T., Muller, E., Kramer, C., Ossenkopf, V., Henkel, C., Deragopian, G., Donnelly, S., \& Ladd, E. F. 2009, MNRAS, 395, 1021

Longmore, S. N., Bally, J., Testi, L., Purcell, C. R., Walsh, A. J., Bressert, E., Pestalozzi, M., Molinari, S., Ott, J., Cortese, L., Battersby, C., Murray, N., Lee, E., Kruijssen, J. M. D., Schisano, E., \& Elia, D. 2013a, MNRAS, 429, 987

Longmore, S. N., Kruijssen, J. M. D., Bally, J., Ott, J., Testi, L., Rathborne, J., Bastian, N., Bressert, E., Molinari, S., Battersby, C., \& Walsh, A. J. 2013b, MNRAS, 433, L15

Longmore, S. N., Rathborne, J., Bastian, N., Alves, J., Ascenso, J., Bally, J., Testi, L., Longmore, A., Battersby, C., Bressert, E., Purcell, C., Walsh, A., Jackson, J., Foster, J., Molinari, S., Meingast, S., Amorim, A., Lima, J., Marques, R., Moitinho, A., Pinhao, J., Rebordao, J., \& Santos, F. D. 2012, ApJ, 746, 117

Martín-Pintado, J., Rizzo, J. R., de Vicente, P., Rodríguez-Fernández, N. J., \& Fuente, A. 2001, ApJ, 548, L65

McMillan, S. L. W., Vesperini, E., \& Portegies Zwart, S. F. 2007, ApJ, 655, L45

Molinari, S., Bally, J., Noriega-Crespo, A., Compiègne, M., Bernard, J. P., Paradis, D., Martin, P., Testi, L., Barlow, M., Moore, T., Plume, R., Swinyard, B., Zavagno, A., Calzoletti, L., Di Giorgio, A. M., Elia, D., Faustini, F., Natoli, P., Pestalozzi, M., Pezzuto, S., Piacentini, F., Polenta, G., Polychroni, D., Schisano, E., Traficante, A., Veneziani, M., Battersby, C., Burton, M., Carey, S., Fukui, Y., Li, J. Z., Lord, S. D., Morgan, L., Motte, F., Schuller, F., Stringfellow, G. S., Tan, J. C., Thompson, M. A., Ward-Thompson, D., White, G., \& Umana, G. 2011, ApJ, 735, L33

Müller, H. S. P., Schlöder, F., Stutzki, J., \& Winnewisser, G. 2005, Journal of Molecular Structure, 742, 215

Müller, H. S. P., Thorwirth, S., Roth, D. A., \& Winnewisser, G. 2001, A\&A, 370, L49

Negueruela, I., Clark, J. S., \& Ritchie, B. W. 2010, A\&A, 516, A78

Portegies Zwart, S. F., McMillan, S. L. W., \& Gieles, M. 2010, ARA\&A, 48, 431

Rathborne, J. M., Longmore, S. N., Jackson, J. M., Alves, J., Bally, J., Bastian, N., Bressert, E., Contreras, Y., Foster, J. B., Garay, G., Kruijssen, J. M. D., Testi, L., \& Walsh, A. J. 2013, submitted 
Redman, M. P., Rawlings, J. M. C., Nutter, D. J., Ward-Thompson, D., \& Williams, D. A. 2002, MNRAS, 337, L17

Rodríguez, L. F. \& Zapata, L. A. 2013, ApJ, 767, L13

Sault, R. J., Teuben, P. J., \& Wright, M. C. H. 1995, in Astronomical Data Analysis Software and Systems IV, ed. R. Shaw, H. Payne, \& J. Hayes, Vol. 77 (San Francisco: ASP Conference Series), 433

Savage, C., Apponi, A. J., Ziurys, L. M., \& Wyckoff, S. 2002, ApJ, 578, 211

Schilke, P., Walmsley, C. M., Pineau Des Forets, G., Roueff, E., Flower, D. R., \& Guilloteau, S. 1992, A\&A, 256, 595

Schöier, F. L., van der Tak, F. F. S., van Dishoeck, E. F., \& Black, J. H. 2005, A\&A, 432, 369

Schuller, F., Menten, K. M., Contreras, Y., Wyrowski, F., Schilke, P., Bronfman, L., Henning, T., Walmsley, C. M., Beuther, H., Bontemps, S., Cesaroni, R., Deharveng, L., Garay, G., Herpin, F., Lefloch, B., Linz, H., Mardones, D., Minier, V., Molinari, S., Motte, F., Nyman, L., Reveret, V., Risacher, C., Russeil, D., Schneider, N., Testi, L., Troost, T., Vasyunina, T., Wienen, M., Zavagno, A., Kovacs, A., Kreysa, E., Siringo, G., \& Weiß, A. 2009, A\&A, 504, 415

van der Tak, F. F. S., Black, J. H., Schöier, F. L., Jansen, D. J., \& van Dishoeck, E. F. 2007, A\&A, 468, 627

Vasyunina, T., Linz, H., Henning, T., Zinchenko, I., Beuther, H., \& Voronkov, M. 2011, A\&A, 527, A88

Walmsley, C. M., Guesten, R., Angerhofer, P., Churchwell, E., \& Mundy, L. 1986, A\&A, 155,129

Wilson, T. L. 1999, Reports on Progress in Physics, 62, 143

Wilson, T. L., Ruf, K., Walmsley, C. M., Martin, R. N., Batrla, W., \& Pauls, T. A. 1982, A\&A, 115, 185

Yusef-Zadeh, F., Wardle, M., Lis, D., Viti, S., Brogan, C., Chambers, E., Pound, M., \& Rickert, M. 2013, Journal of Physical Chemistry A, 117, 9404 
Table 1: Summary of the molecular transitions observed and the detected emission (ordered by increasing excitation energies ${ }^{\mathrm{a}}$.

\begin{tabular}{|c|c|c|c|c|c|c|c|}
\hline \multirow[t]{2}{*}{ Transition } & \multirow{2}{*}{$\begin{array}{c}\text { Frequency } \\
(\mathrm{GHz})\end{array}$} & \multirow{2}{*}{$\begin{array}{r}\mathrm{E}_{u} / k \\
(\mathrm{~K})\end{array}$} & \multirow{2}{*}{$\begin{array}{c}\mathrm{n}_{\text {crit }} \\
\left(\mathrm{cm}^{-3}\right)\end{array}$} & \multicolumn{4}{|c|}{ Peak position } \\
\hline & & & & $\begin{array}{l}\text { Long } \\
\text { (deg) }\end{array}$ & $\begin{array}{l}\text { Lat } \\
(\operatorname{deg})\end{array}$ & $\begin{array}{c}\text { Vel } \\
\left(\mathrm{km} \mathrm{s}^{-1}\right)\end{array}$ & $\begin{array}{l}\mathrm{T}_{m b} \\
(\mathrm{~K})\end{array}$ \\
\hline $\mathrm{H}^{13} \mathrm{CO}^{+}(1-0)$ & 86.754330 & 4.16 & $2 \times 10^{5}$ & 0.248 & 0.004 & 36.68 & 0.39 \\
\hline $\mathrm{HN}^{13} \mathrm{C}(1-0)$ & 87.090859 & 4.18 & $3 \times 10^{5}$ & 0.248 & 0.007 & 36.35 & 0.58 \\
\hline $\mathrm{C}_{2} \mathrm{H}(1-0) 3 / 2-1 / 2$ & 87.316925 & 4.19 & $2 \times 10^{5}$ & 0.243 & 0.007 & 38.69 & 1.00 \\
\hline $\mathrm{HCN}(1-0)$ & 88.631847 & 4.25 & $3 \times 10^{6}$ & 0.248 & 0.007 & 47.51 & 4.67 \\
\hline $\mathrm{HCO}^{+}(1-0)$ & 89.188526 & 4.28 & $2 \times 10^{5}$ & 0.246 & 0.007 & 44.48 & 3.31 \\
\hline $\mathrm{HNC}(1-0)$ & 90.663572 & 4.35 & $3 \times 10^{5}$ & 0.248 & 0.009 & 41.21 & 2.91 \\
\hline $\mathrm{N}_{2} \mathrm{H}^{+}(1-0)$ & 93.173772 & 4.47 & $3 \times 10^{5}$ & 0.248 & 0.007 & 43.32 & 3.53 \\
\hline $\mathrm{SiO}(2-1)$ & 86.847010 & 6.25 & $3 \times 10^{5}$ & 0.246 & 0.004 & 40.34 & 1.07 \\
\hline${ }^{13} \mathrm{CS}(2-1)$ & 92.494303 & 6.66 & $3 \times 10^{5}$ & 0.226 & -0.001 & 34.75 & 0.37 \\
\hline${ }^{13} \mathrm{C}^{34} \mathrm{~S}(2-1)$ & 90.926036 & 7.05 & $4 \times 10^{5}$ & - & - & - & - \\
\hline $\mathrm{HNCO} 4(0,4)-3(0,3)$ & 87.925238 & 10.55 & $1 \times 10^{6}$ & 0.246 & 0.007 & 36.86 & 2.52 \\
\hline $\mathrm{CH}_{3} \mathrm{CN} 5(1)-4(1)$ & 91.985316 & 20.39 & $4 \times 10^{5}$ & 0.243 & 0.004 & 33.13 & 0.87 \\
\hline $\mathrm{HC}_{3} \mathrm{~N}(10-9)$ & 90.978989 & 24.01 & $5 \times 10^{5}$ & 0.246 & 0.007 & 37.52 & 2.98 \\
\hline $\mathrm{HC}^{13} \mathrm{CCN}(10-9)$ & 90.593059 & 24.37 & $1 \times 10^{6}$ & - & - & - & - \\
\hline $\mathrm{HCN}(3-2)$ & 265.886434 & 25.52 & $7 \times 10^{7}$ & 0.243 & 0.007 & 43.30 & 3.70 \\
\hline $\mathrm{HCO}^{+}(3-2)$ & 267.557619 & 25.68 & $4 \times 10^{6}$ & 0.243 & 0.007 & 43.00 & 1.74 \\
\hline $\mathrm{HNC}(3-2)$ & 271.981142 & 26.11 & $8 \times 10^{6}$ & 0.258 & 0.019 & 40.80 & 1.21 \\
\hline $\mathrm{N}_{2} \mathrm{H}^{+}(3-2)$ & 279.511701 & 26.83 & $3 \times 10^{6}$ & 0.243 & 0.007 & 38.40 & 3.16 \\
\hline $\mathrm{SiO}(5-4)$ & 217.104980 & 31.26 & $5 \times 10^{6}$ & 0.241 & 0.007 & 39.07 & 0.56 \\
\hline $\mathrm{HCN}(4-3)$ & 354.505477 & 42.53 & $3 \times 10^{8}$ & 0.243 & 0.004 & 40.70 & 1.55 \\
\hline $\mathrm{HCO}^{+}(4-3)$ & 356.734288 & 42.80 & $9 \times 10^{6}$ & 0.248 & 0.004 & 35.00 & 0.84 \\
\hline $\mathrm{HNC}(4-3)$ & 362.630303 & 43.51 & $8 \times 10^{7}$ & 0.251 & 0.009 & 37.60 & 0.84 \\
\hline $\mathrm{N}_{2} \mathrm{H}^{+}(4-3)$ & 372.672509 & 44.71 & $8 \times 10^{6}$ & 0.241 & 0.007 & 40.00 & 0.37 \\
\hline HNCO $4(1,3)-3(1,2)$ & 88.239027 & 53.86 & $1 \times 10^{6}$ & - & - & - & - \\
\hline $\mathrm{H} 41 \alpha$ & 92.034475 & & & - & - & - & - \\
\hline
\end{tabular}

${ }^{a}$ Excitation energies and critical densities $\left(\mathrm{E}_{u}\right.$ and $\mathrm{n}_{\text {crit }}$ ) were calculated using values from the Leiden Atomic and Molecular Database (LAMDA; Schöier et al. 2005) and Cologne Database for Molecular Spectroscopy (CDMS; Müller et al. 2001, 2005) assuming a gas temperature of $20 \mathrm{~K}$. 

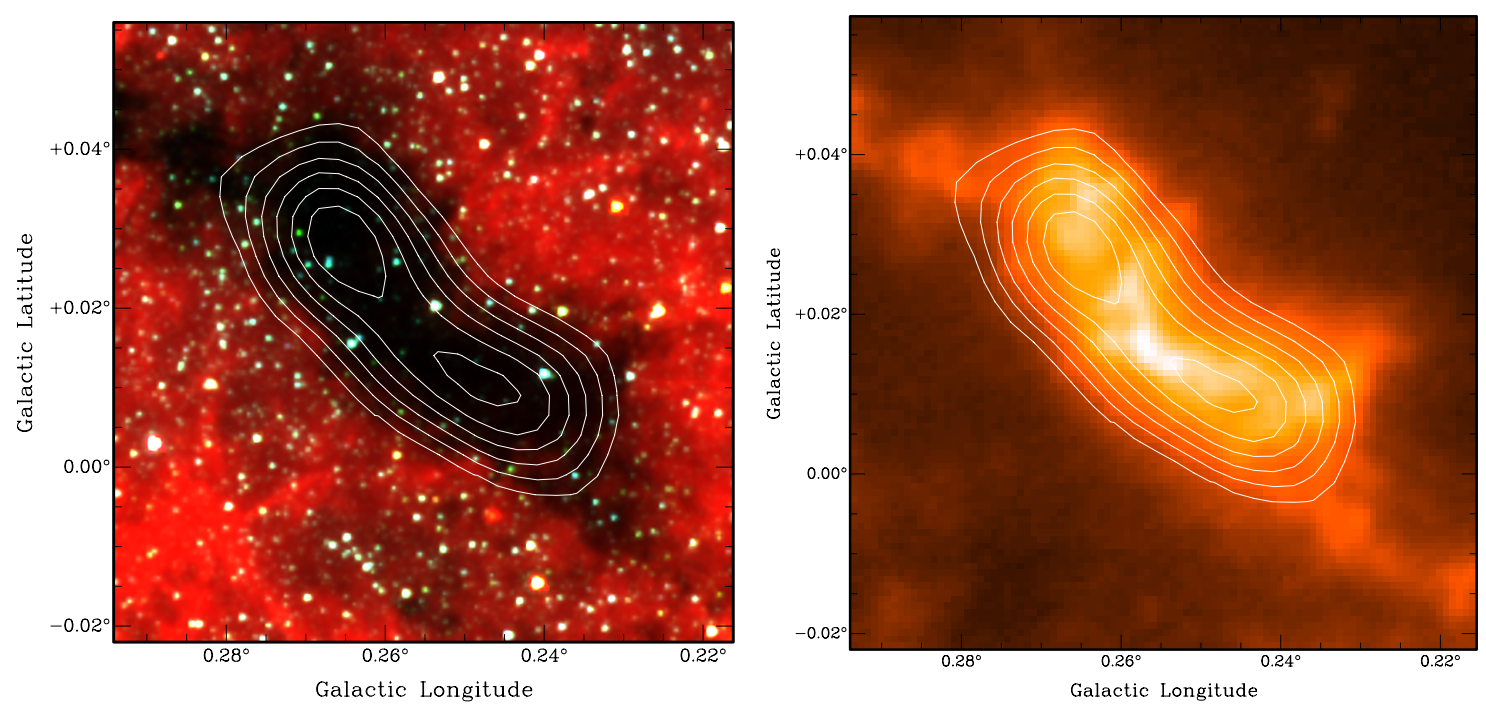

Fig. 1. - Continuum images toward G0.253+0.016 (left: Spitzer/IRAC three colour and right: JCMT $450 \mu \mathrm{m}$; Longmore et al. 2012) overlaid with the HNCO 4(0,4)-3(0,3) integrated intensity image (this image was generated by integrating the emission in the range $-25 \mathrm{~km} \mathrm{~s}^{-1}<\mathrm{V}_{L S R}<60 \mathrm{~km} \mathrm{~s}^{-1}$; see section 3.4 contours levels are from 10 to $90 \%$ of the peak in steps of $10 \%$. The $450 \mu \mathrm{m}$ emission ranges from $10 \mathrm{Jy}^{\text {beam }}{ }^{-1}$ at the clump's edge to $50 \mathrm{Jy}$ beam $^{-1}$ at the central peak). The clump is seen as an extinction feature in the IR but is a strong emitter at sub-mm/mm wavelengths. Overall the morphology of the molecular line emission matches well the mid-IR extinction and sub-mm dust continuum emission. 

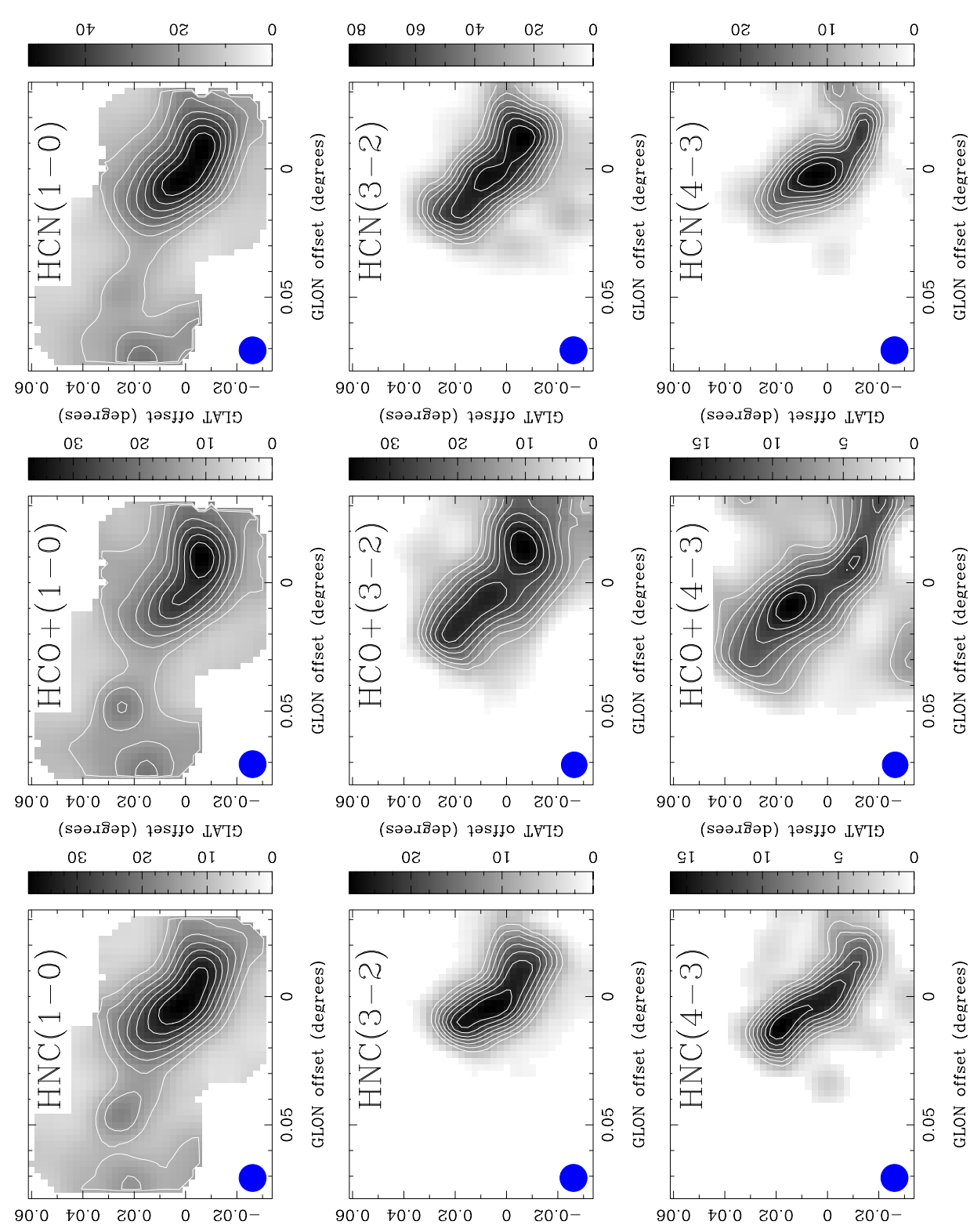

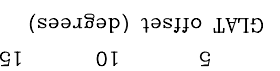

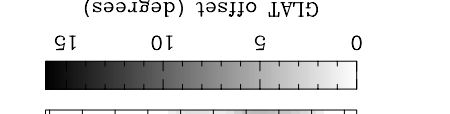

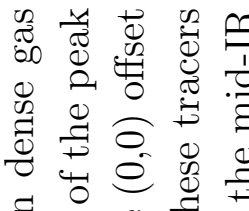

寻

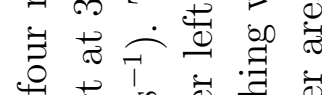

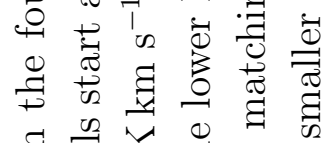

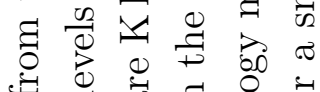

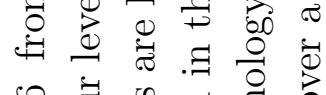

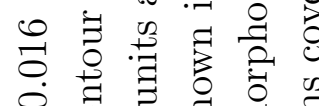

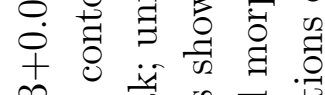

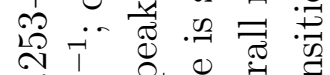

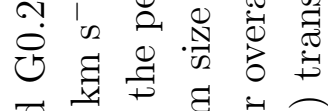

चี

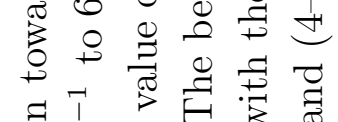

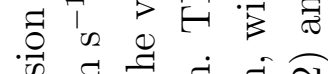

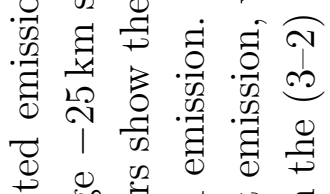

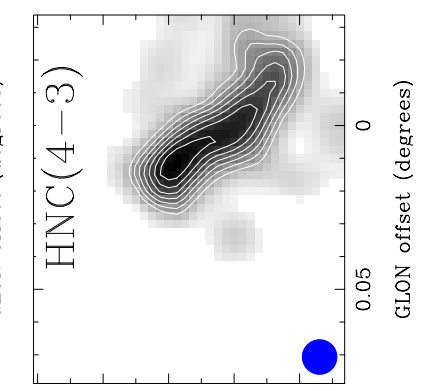

$20^{\circ} 0 \quad 70^{\circ} 0 \quad 20^{\circ} 0 \quad 0 \quad 20^{\circ} 0$

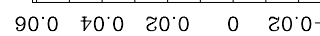

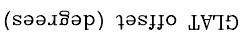

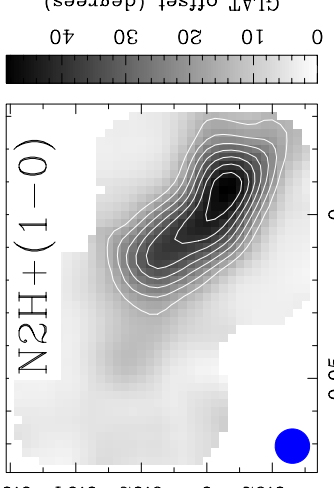

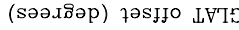

08

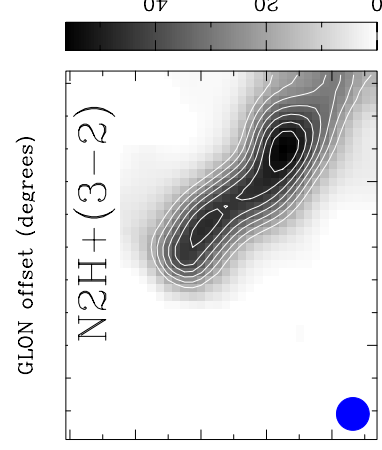

$90^{\circ} 0 \quad 70^{\circ} 0 \quad 80^{\circ} 0 \quad 0 \quad 80^{\circ}$

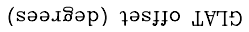

$90^{\circ} 0 \quad 10^{\circ} 0 \quad \approx 0^{\circ} 0 \quad 0 \quad 20^{\circ} 0$

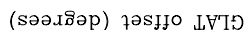

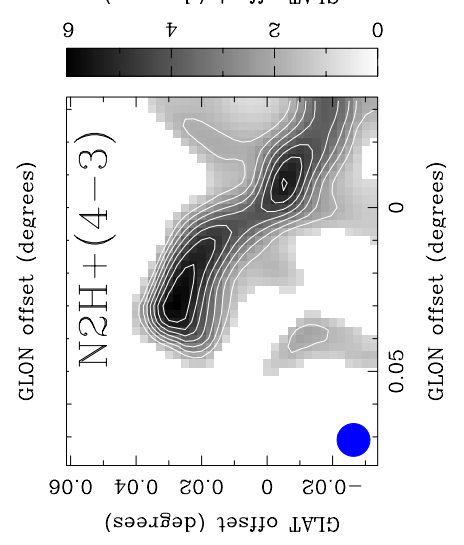

兽

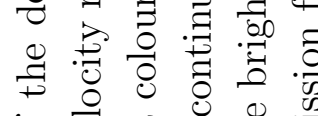

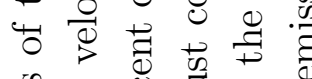

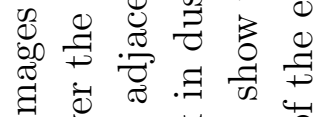

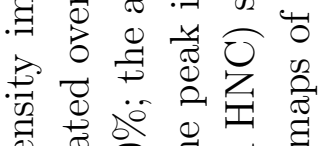

矛

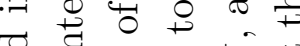

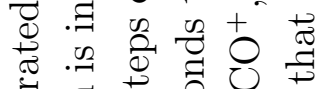

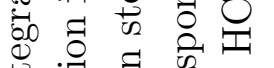

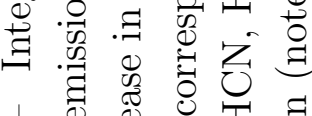

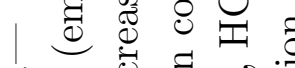

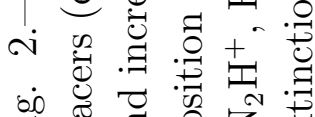

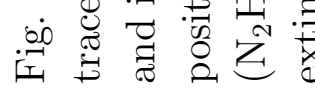



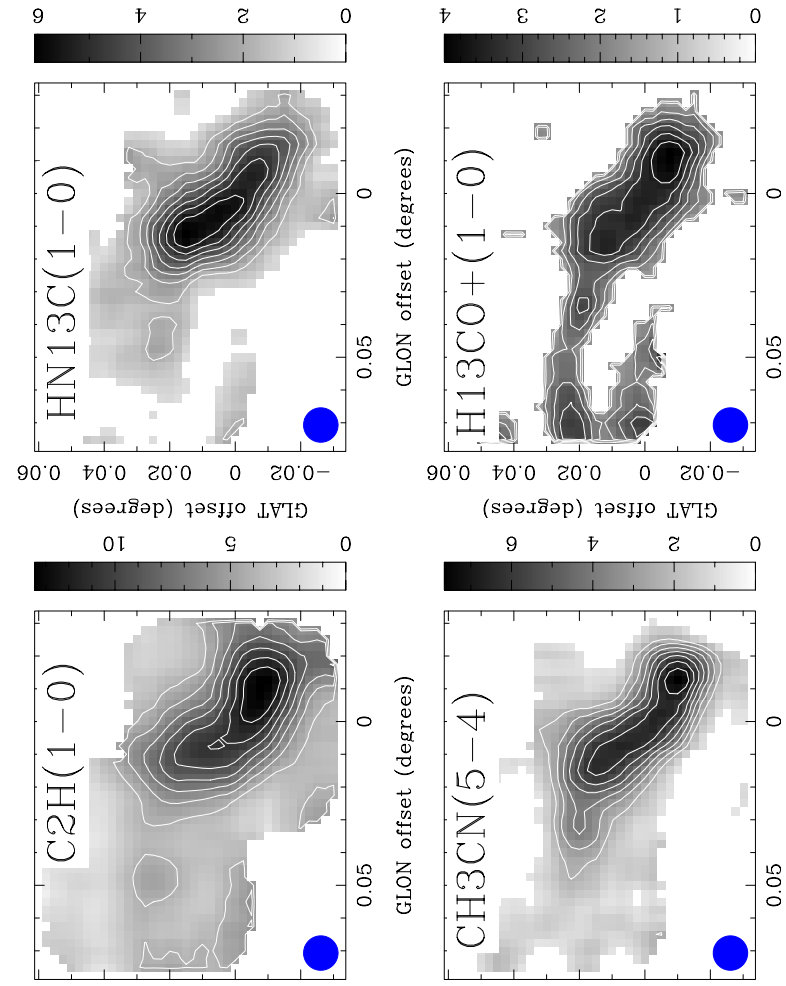

$90^{\circ} 0 \quad 10.080 .0 \quad 0 \quad 80^{\circ} 0-$

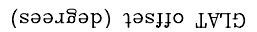

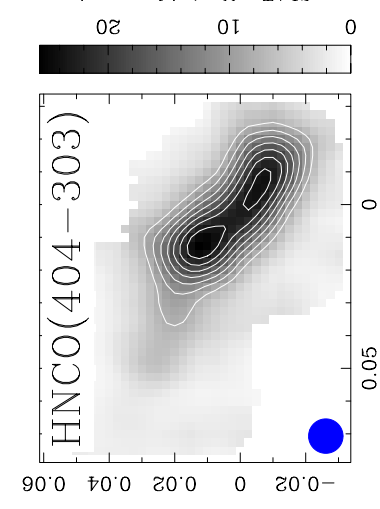

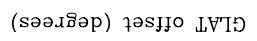

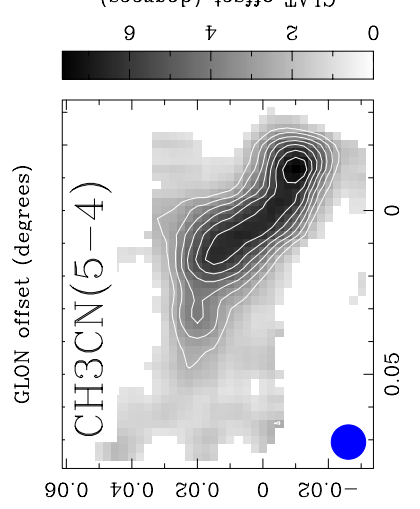

$20^{\circ} 0 \begin{gathered}1 \\ 1\end{gathered}$

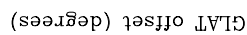

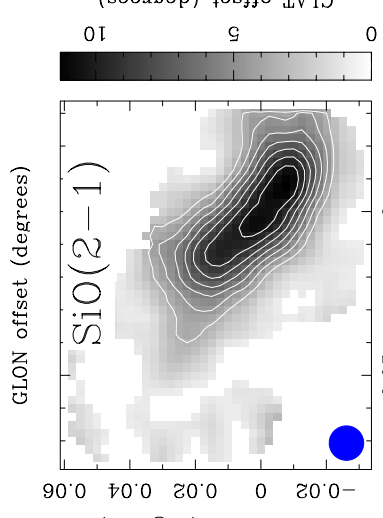

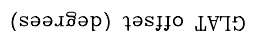

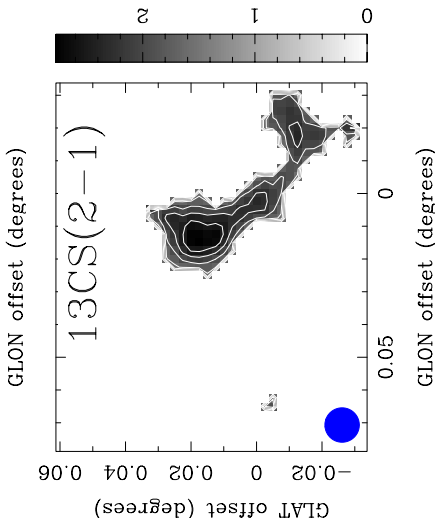

$0 z$ or

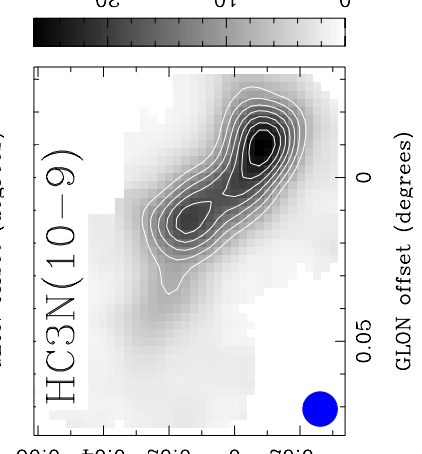

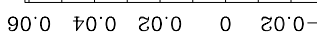

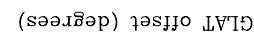

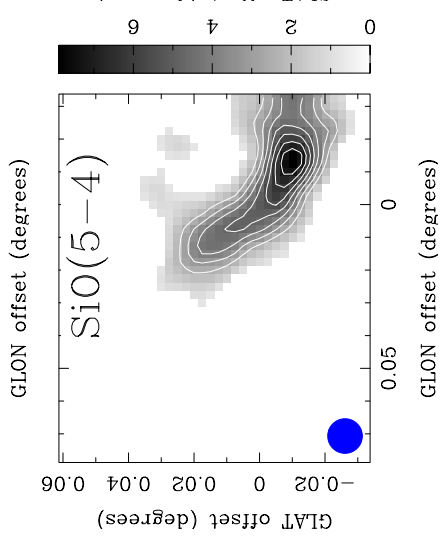

శ్రి

㐘

뭉

झु

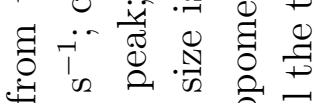

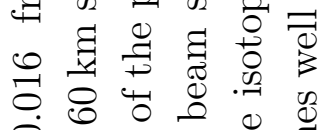

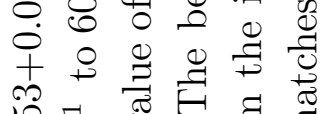

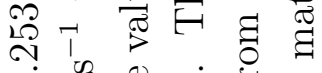

¿

茪

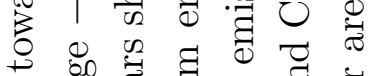

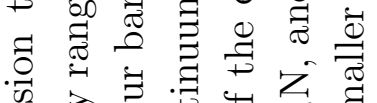

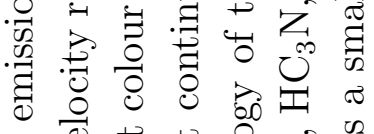

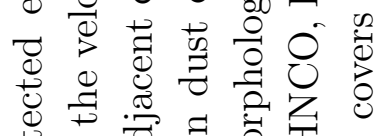

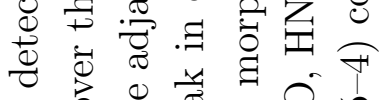

ఫ

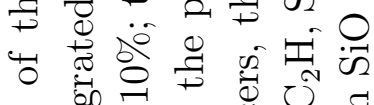

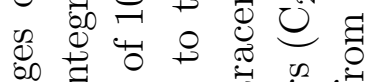

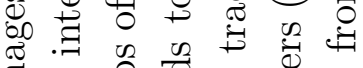

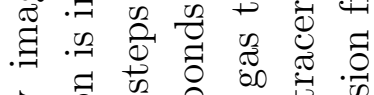

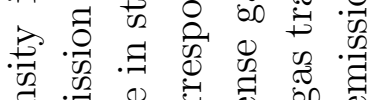

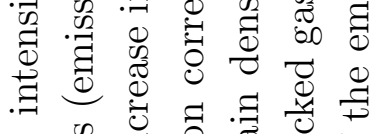

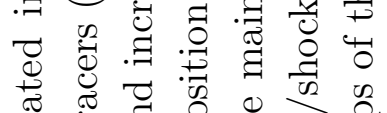

范

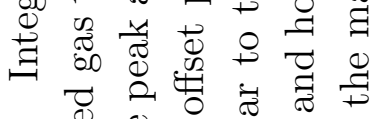

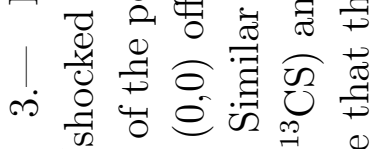

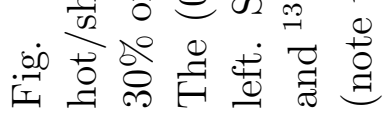




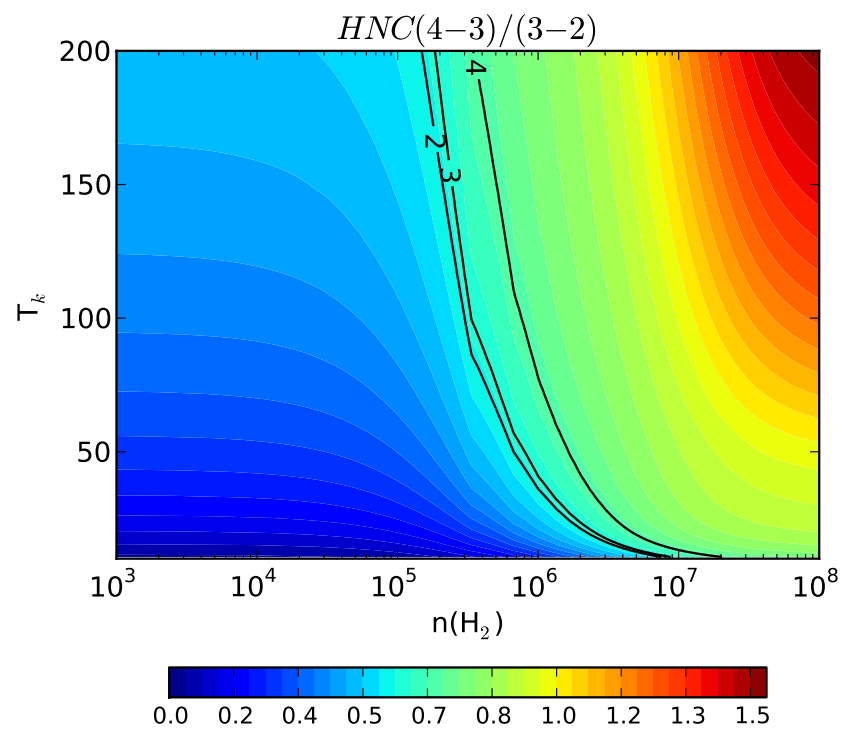

Fig. 4.- The predicted HNC line ratios as a function of volume density and kinetic temperature. This plot was generated using RADEX, a radiative transfer model under the assumption of non-LTE conditions. The solid black lines mark the values of the ratio calculated from the data toward three of the 5 positions within G0.253+0.016 (P2, P3, and $\mathrm{P} 4)$. While we are unable to constrain the kinetic temperature, we measure gas densities of $\sim 10^{6} \mathrm{~cm}^{-3}$. 

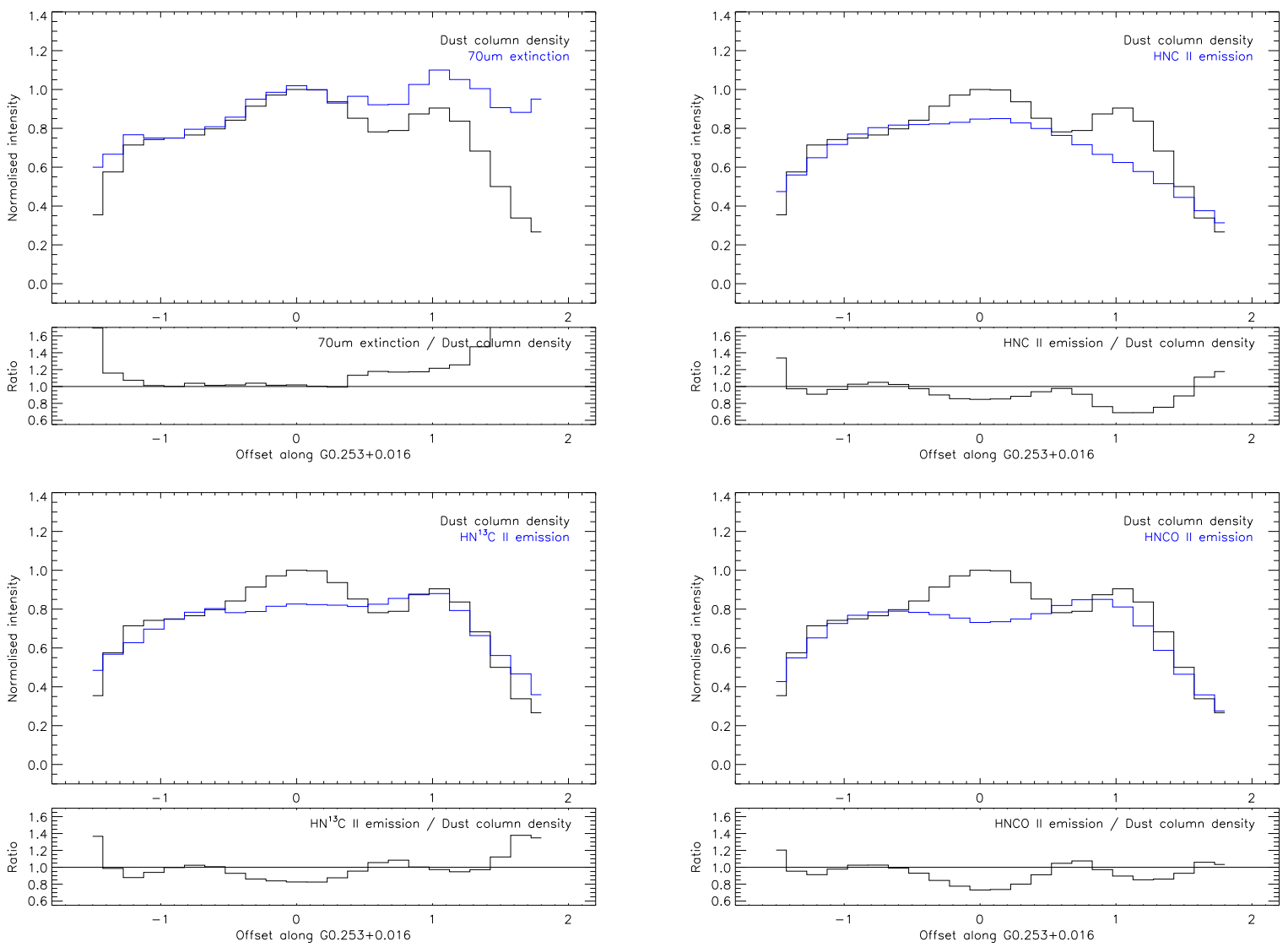

Fig. 5.- Normalised dust column density profile along the major axis of G0.253+0.016 overlaid with $70 \mu \mathrm{m}$ extinction profile (left upper panel), $\mathrm{HNC}$ (right upper panel), $\mathrm{HN}^{13} \mathrm{C}$ (left lower panel) and HNCO (right lower panel) integrated intensity emission profiles (in all cases the emission has been normalised to match the normalised dust column density toward the clump's edges). Under each panel is the ratio of the normalised profiles. Overall, the dust column density profile is matched well by the $70 \mu \mathrm{m}$ extinction profile (there is relatively more extinction toward the upper portion of the clump, positive offsets, compared to the centre and lower portion). In contrast, the line emission profiles appear anti-correlated with the dust column density toward the clump's centre, regardless of the gas tracer (i.e. $\mathrm{HNC}, \mathrm{HN}^{13} \mathrm{C}$, or $\mathrm{HNCO}$; these three molecules were selected to represent the emission from the dense gas tracers, isotopolgues, and hot gas tracers respectively). If the line emission is optically thin, then its profile ought to match the dust column density profile. Given the density of the clump, the emission from HNC is likely optically thick. However, the isotopologues and hot gas tracers (which are less likely to be optically thick) also show an absence of emission toward the clump's centre. Thus, if they are indeed optically thin, then we interpret this anti-correlation to arise due to gas depletion in the clump's cold interior. 

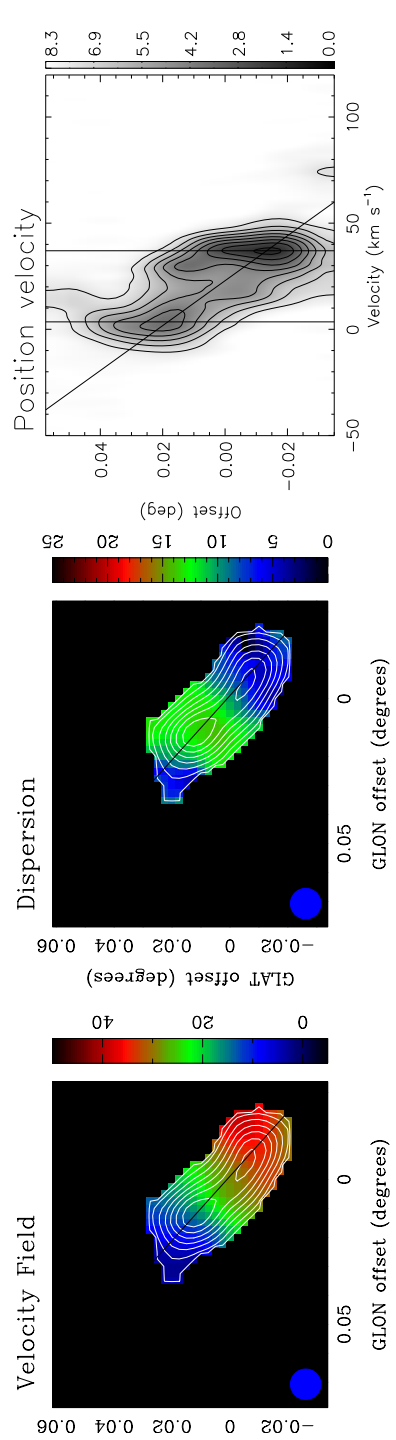

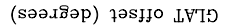

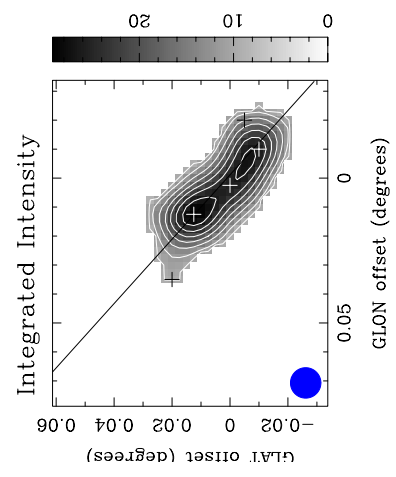

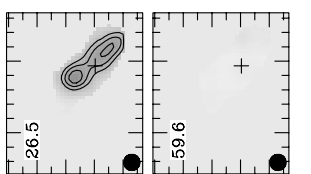
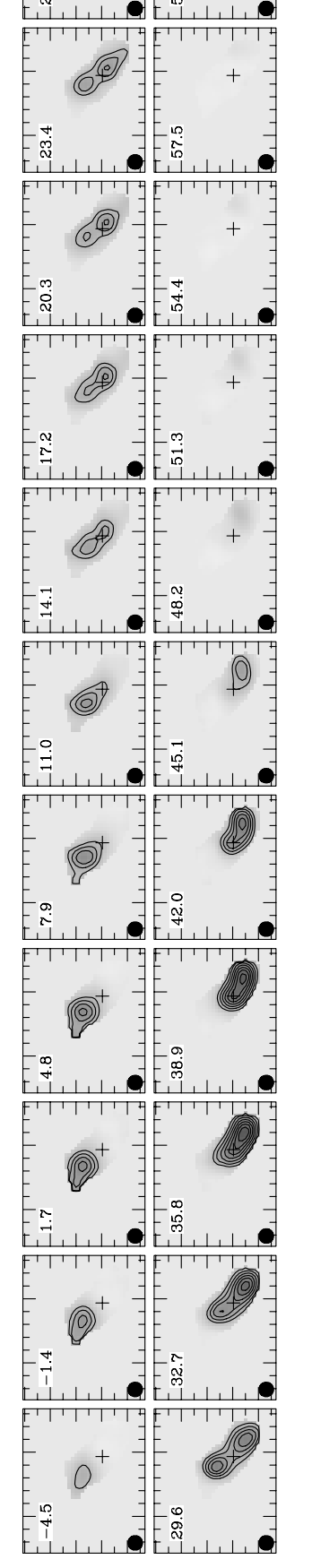

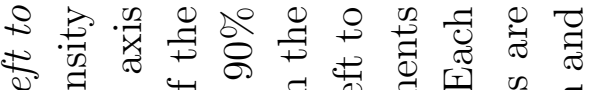
હ हీ. ३) क ¿ द्व ई

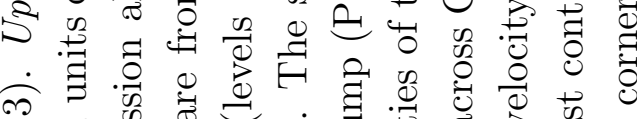
仓

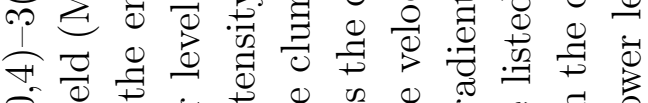

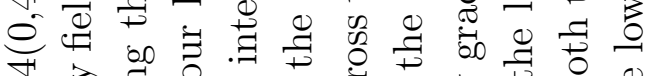
○一

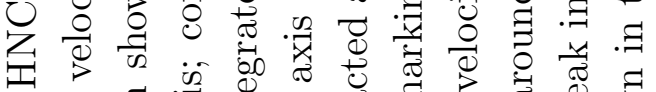
பี 范

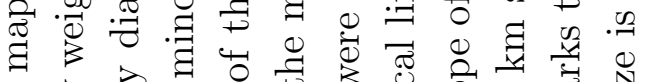

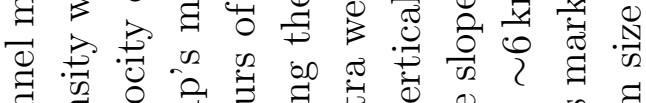

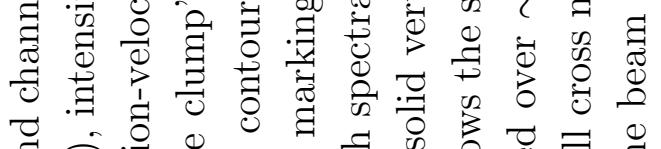

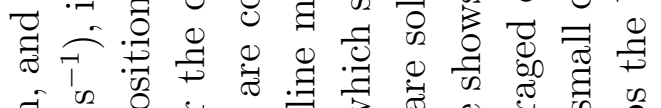

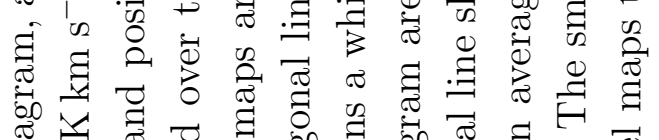
胥

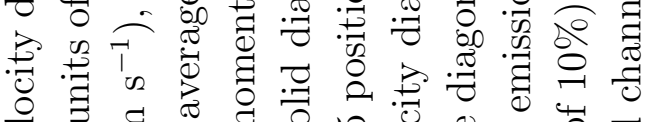

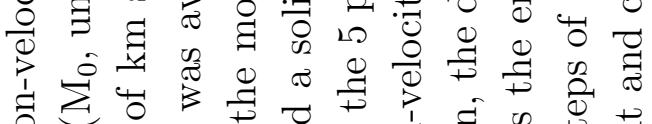

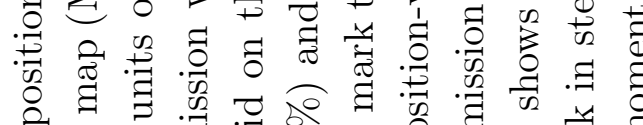

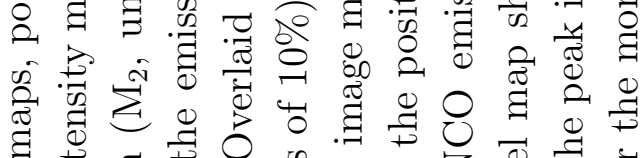
घ.

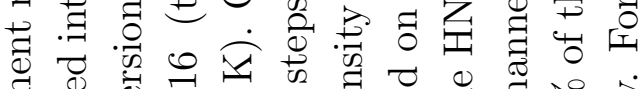

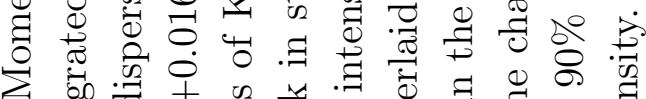

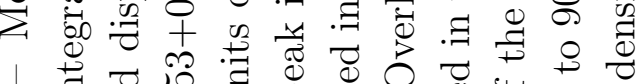

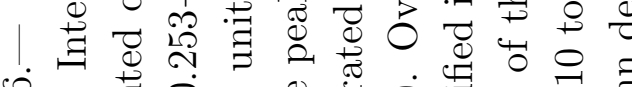

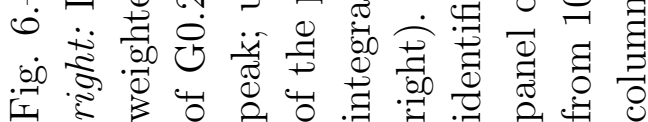




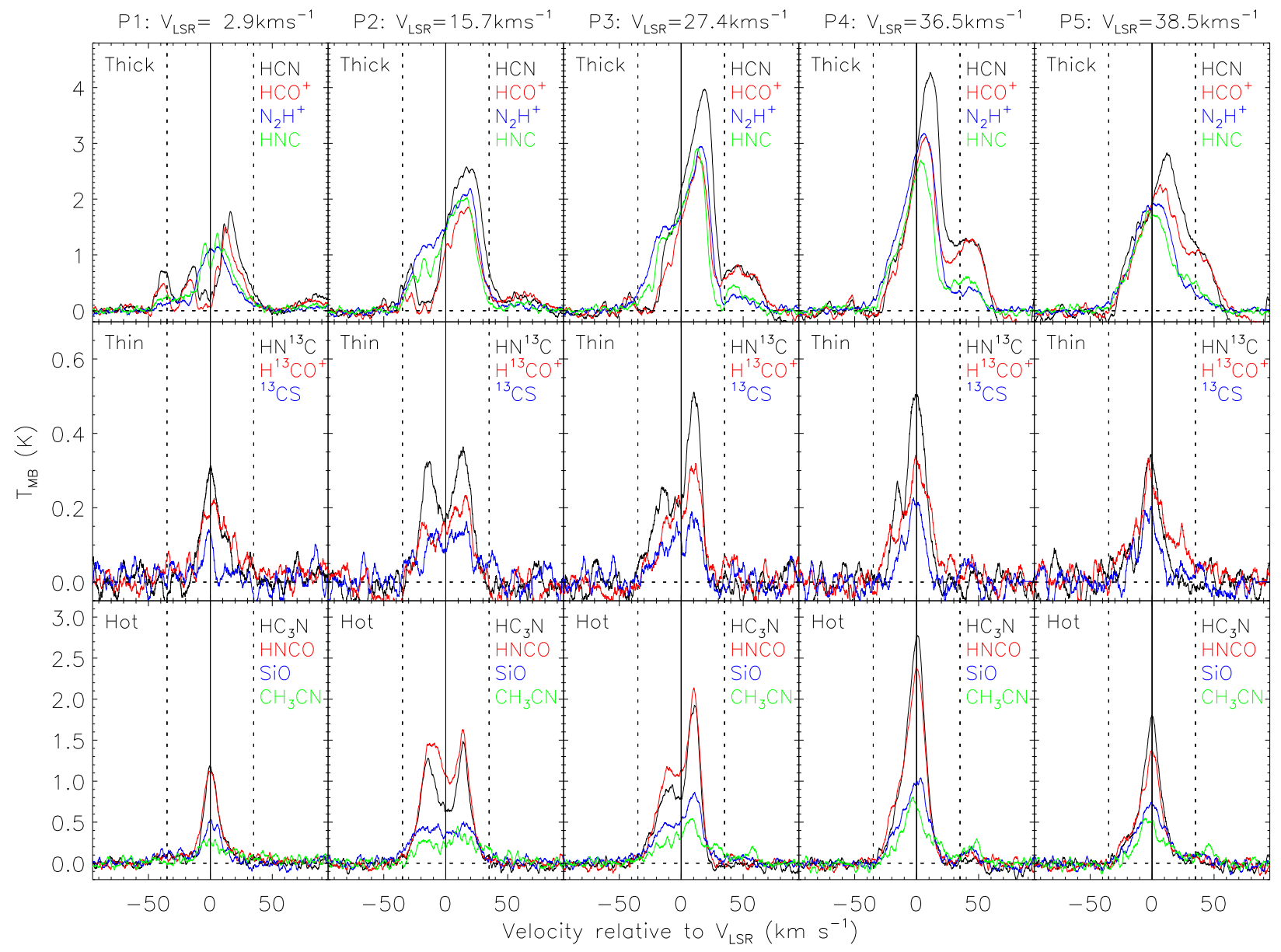

Fig. 7.- Spectra toward 5 positions across G0.253+0.016. The spectra were extracted at the positions marked on the integrated intensity image shown in Figure 6 and show details of the kinematics across the clump. The molecules were grouped based on whether they typically trace emission that is likely to be optically thick (upper panels, $\mathrm{HCN}, \mathrm{HCO}^{+}, \mathrm{N}_{2} \mathrm{H}^{+}$and $\mathrm{HNC}$ ), optically thin (middle panels, $\mathrm{HN}^{13} \mathrm{C} \mathrm{H}^{13} \mathrm{CO}^{+}$, and ${ }^{13} \mathrm{CS}$ ), or from hot/shocked gas (lower panels, $\mathrm{HC}_{3} \mathrm{~N}, \mathrm{HNCO}, \mathrm{SiO}$, and $\mathrm{CH}_{3} \mathrm{CN}$ ). The solid vertical line marks the derived $\mathrm{V}_{L S R}$ of the emission at that location, derived from the HNCO intensity weighted velocity field (the $\mathrm{V}_{L S R}$ is marked at the top of each column). The vertical dotted lines delineate the velocity range we attribute to the emission from G0.253+0.016 $\left( \pm 35 \mathrm{~km} \mathrm{~s}^{-1}\right.$ around the derived $\left.\mathrm{V}_{L S R}\right)$. We find that the spectra from the optically thin tracers and those from the hot/shocked gas have similar profiles. In contrast, spectra from the optically thick gas tracers show that toward all positions the emission peaks red-ward of the other species and of the derived $\mathrm{V}_{L S R}$. 


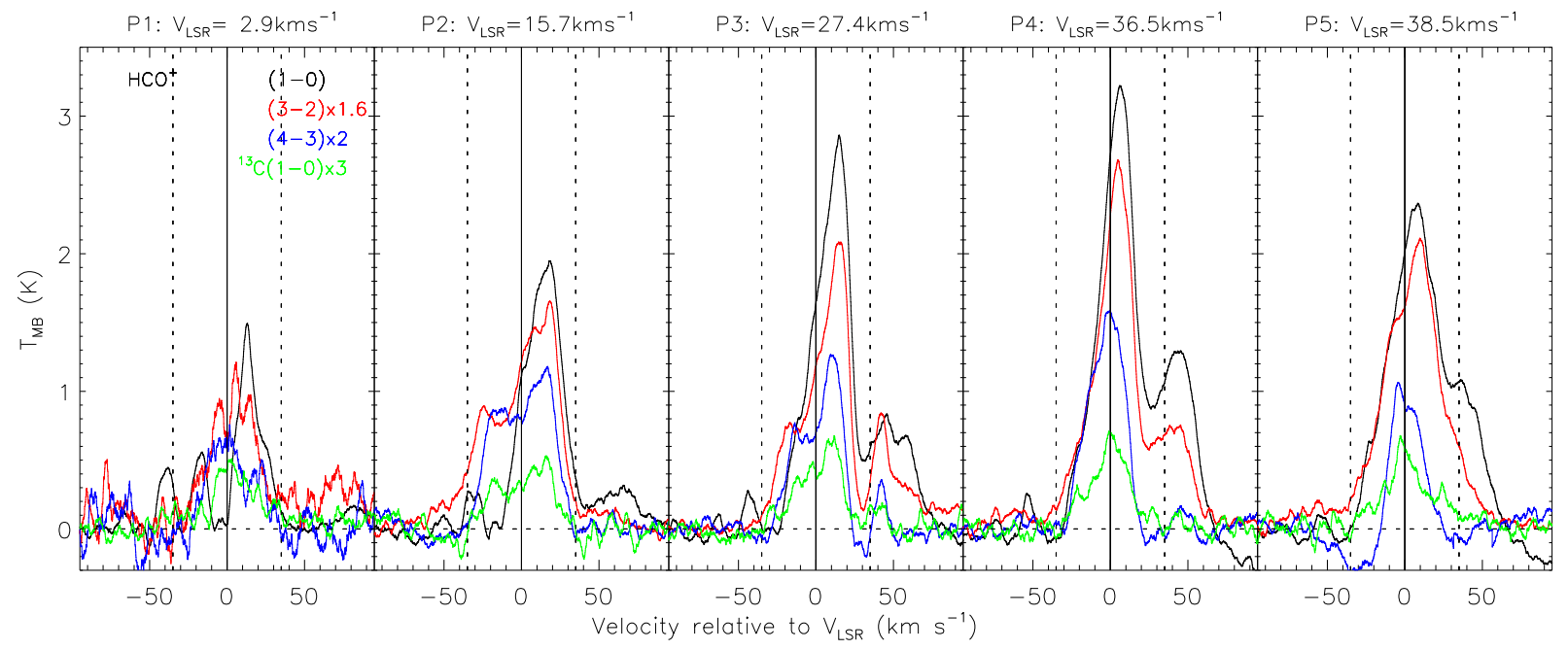

Fig. 8. - $\mathrm{HCO}^{+}$spectra from different transitions toward 5 positions across G0.253+0.016. The spectra were extracted at the positions marked on the integrated intensity image shown in Figure 6. The solid vertical line marks the derived $\mathrm{V}_{L S R}$ of the emission at that location, derived from the HNCO intensity weighted velocity field (the $\mathrm{V}_{L S R}$ is marked at the top of each column). The vertical dotted lines delineate the velocity range we attribute to the emission from $\mathrm{G} 0.253+0.016\left( \pm 35 \mathrm{~km} \mathrm{~s}^{-1}\right.$ around the derived $\left.\mathrm{V}_{L S R}\right)$. Because the molecular transitions are tracers of material at different critical densities, their apparent shift to more central velocities in the higher density gas, is indicative of a density and velocity gradient of material that is centrally condensed. 


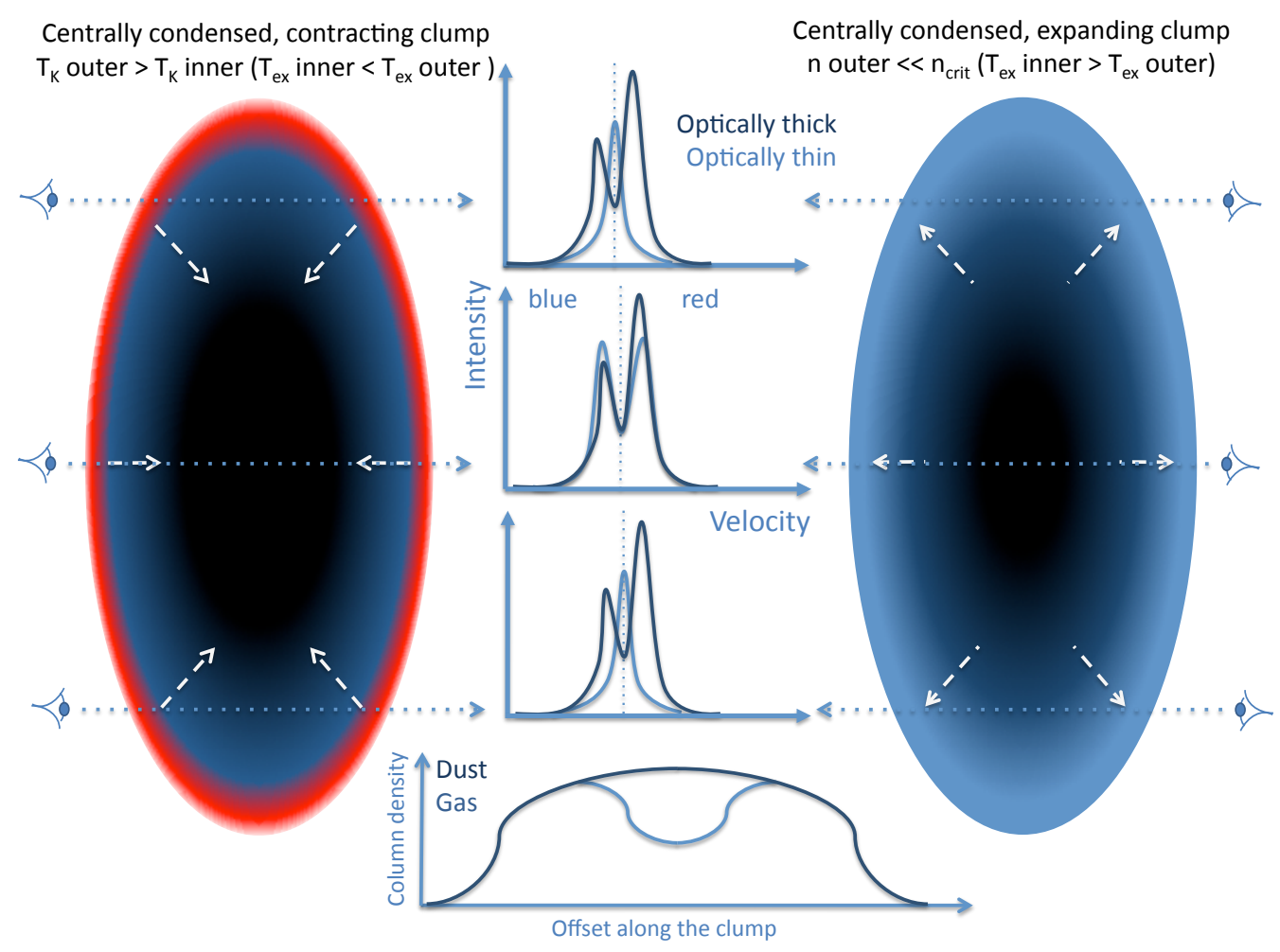

Fig. 9.- Two alternative models for G0.253+0.016, consistent with both the apparent lack of molecular line emission at the central column density peak, and with the observed redblue asymmetry in the optically thick molecular lines: externally heated, collapsing (left, Baked Alaska) vs sub-thermal excitation, expanding (right, P Cygni). In both scenarios the clump has a density gradient that increases toward its centre (white arrows show the gas motion). For an externally heated collapsing clump, the optically thick gas will show a red asymmetry in its line profile: the red velocity component comes from the warmer and brighter $\tau=1$ surface at the front of the cloud, while the blue component comes from the cooler and fainter $\tau=1$ surface toward the centre. In contrast, if the gas is expanding, then the brighter emission at red-shifted velocities arises from the interior of the cloud which has a significantly higher excitation temperature than the exterior portion. Given that the observed dust temperature implies that the clump is externally heated, this scenario requires sub-thermal excitation in the outer edges $\left(\mathrm{n} \ll \mathrm{n}_{\text {crit }}\right)$. In both cases, emission from an optically thin tracer will peak at the clump's $V_{L S R}$. However, if the gas is depleted in the clumps' centre, then the profile from the optically thin tracer will instead show two velocity components that peak in velocity either side of the cloud's $\mathrm{V}_{L S R}$. The distribution of dust and gas emission will not be correlated toward the cloud's centre; instead, the gas will be deficient compared to the dust toward the centre. 
6. Appendix : online only material 

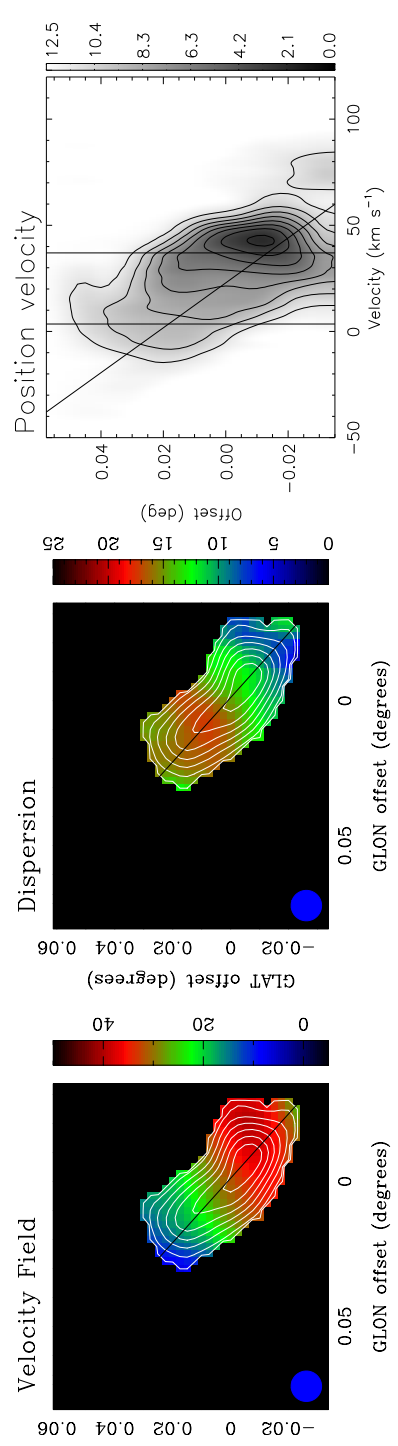

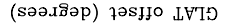

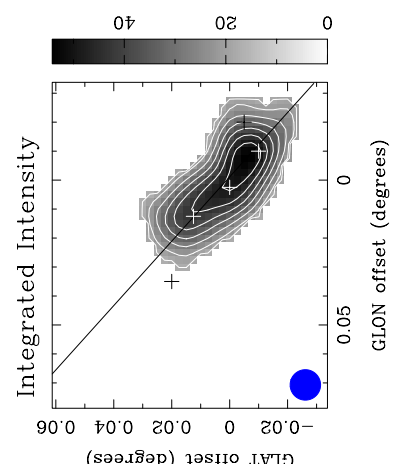

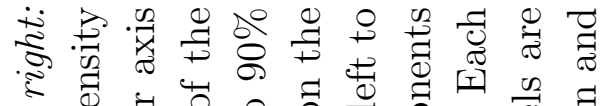
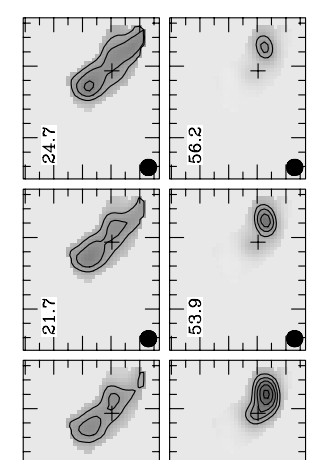

$=\infty$
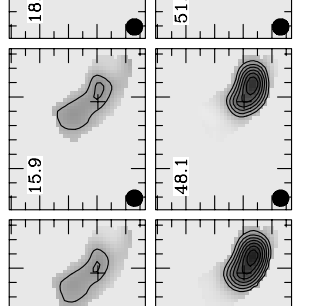

=욜
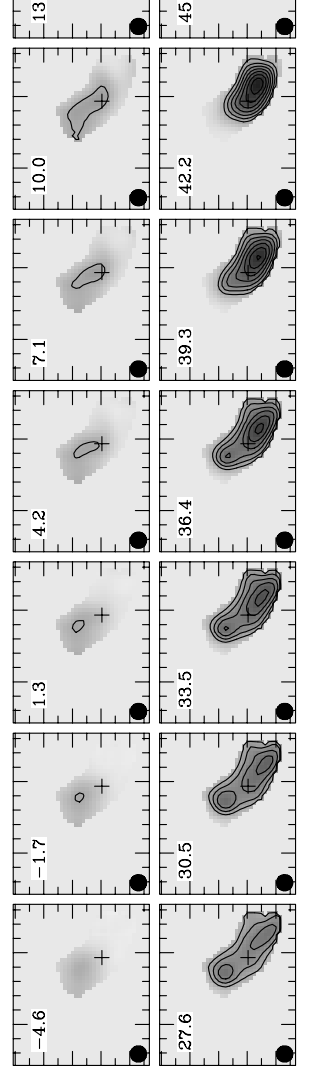

¿

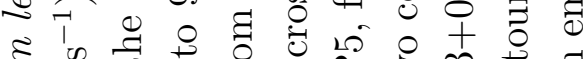
s.

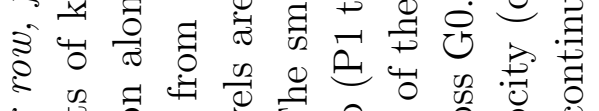

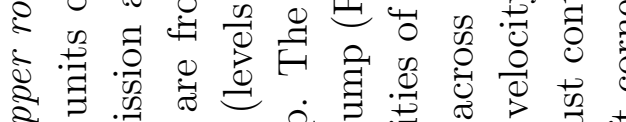

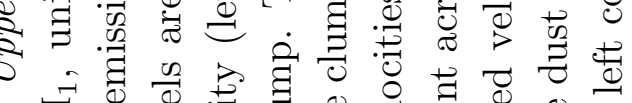

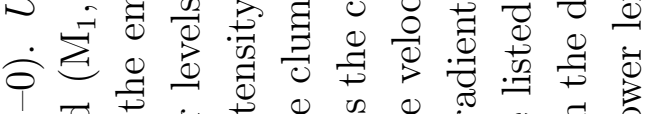

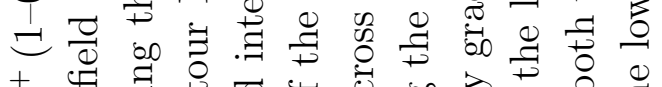

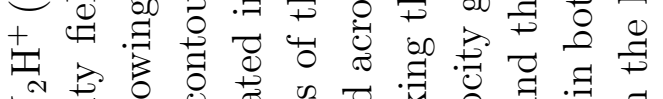
乙

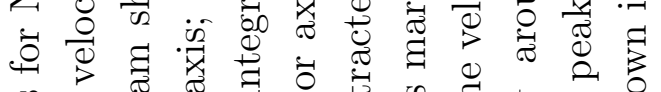
蛋

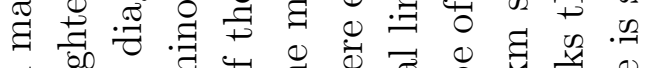

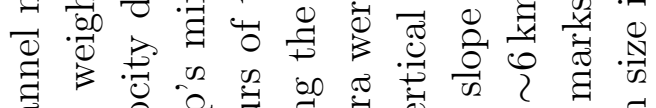

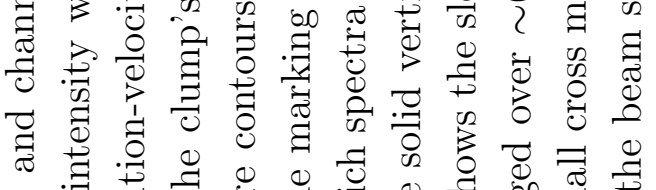

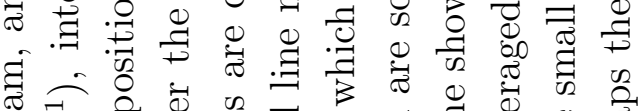

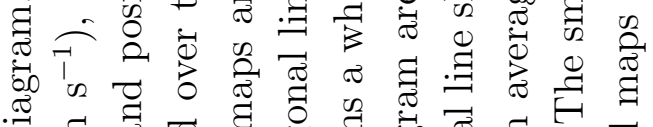
胥

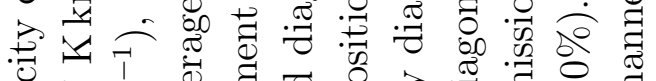

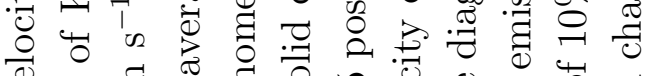

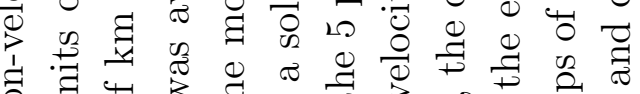

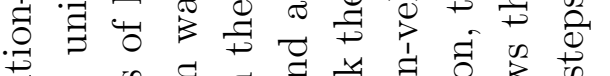

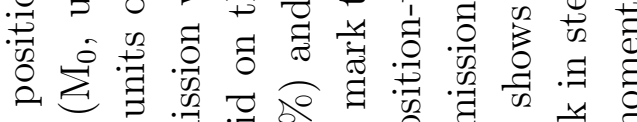

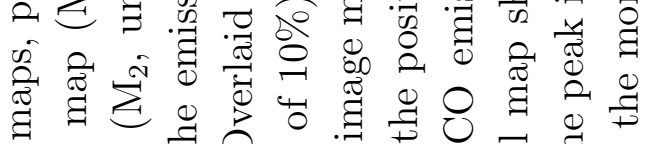

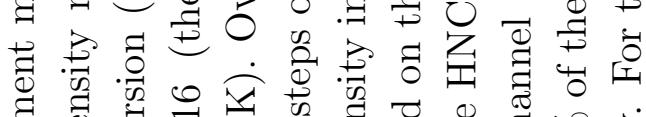

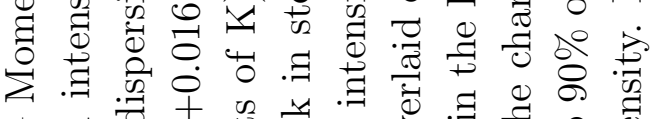

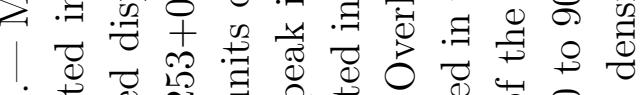

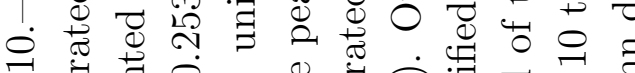

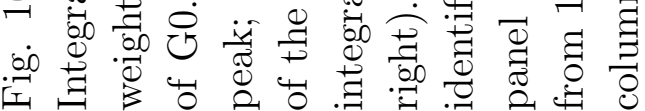

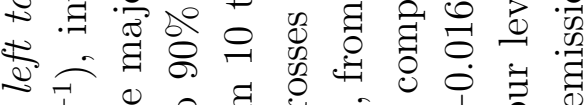



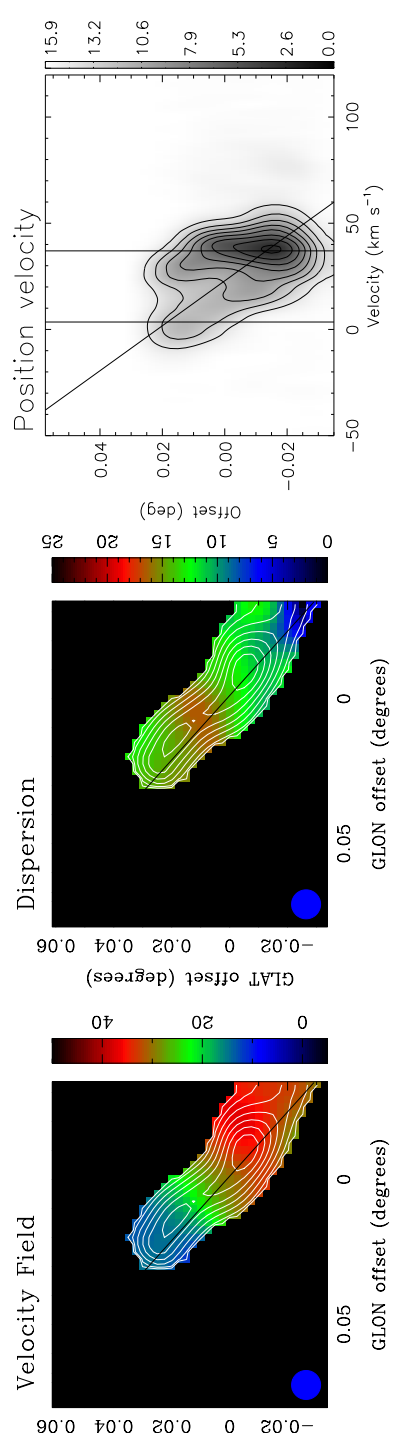

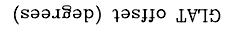

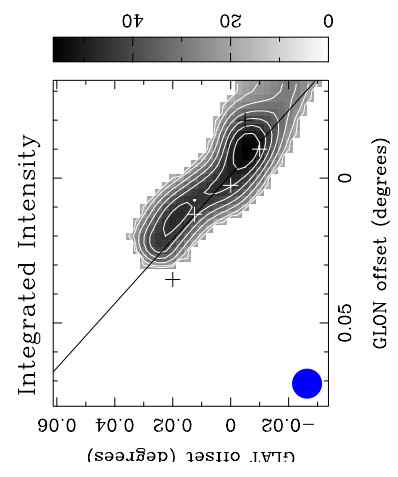

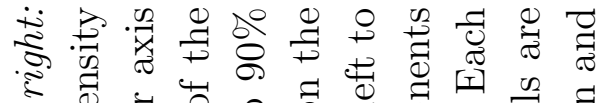
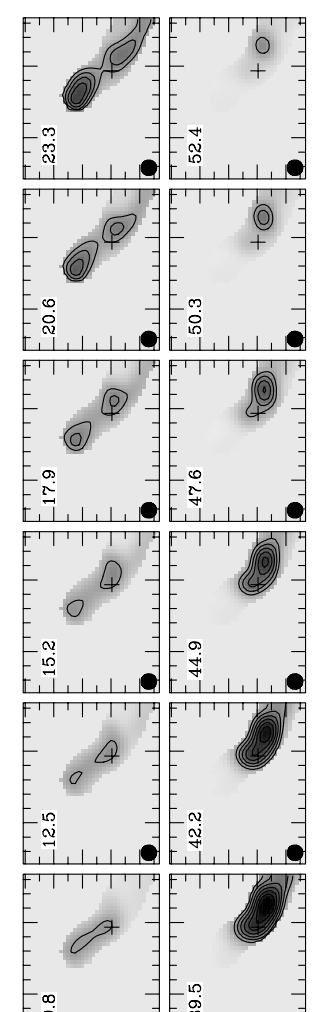

-
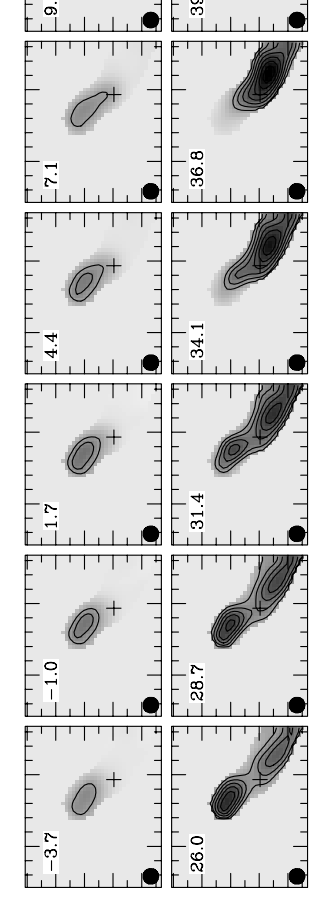

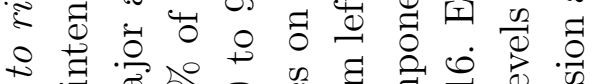

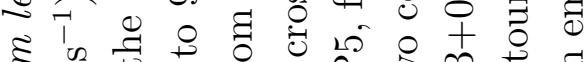

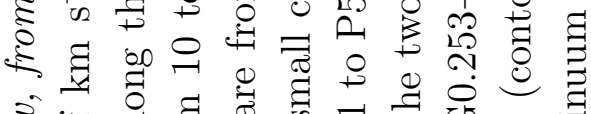

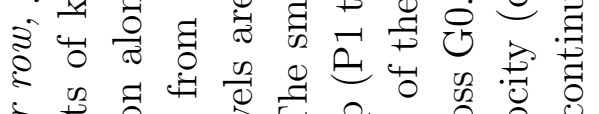

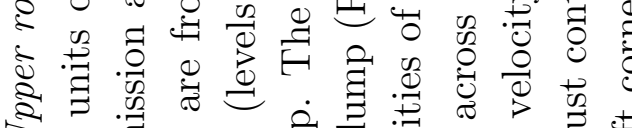
$S=\ddot{g}$ क

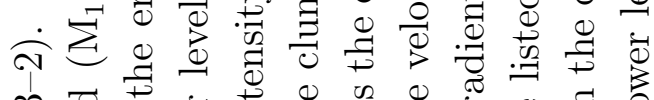
అ

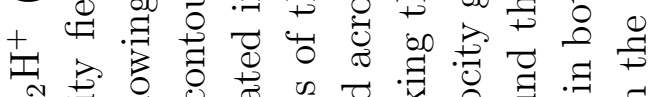
资 馬 号

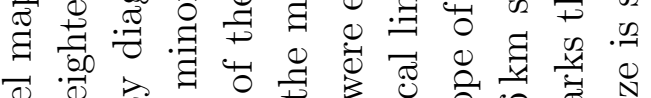

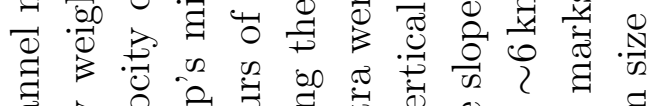

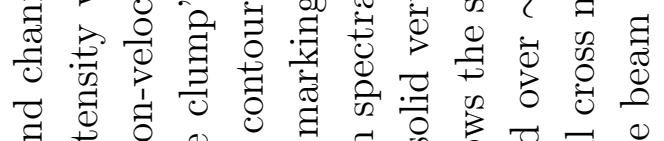

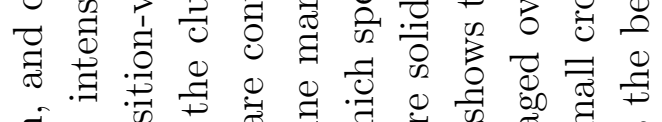

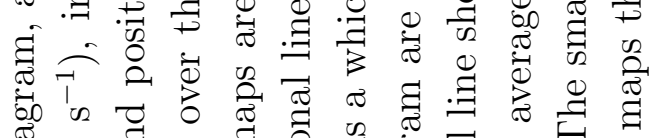
胥

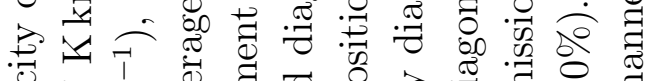
ن

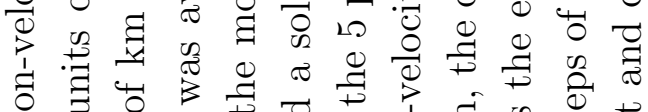

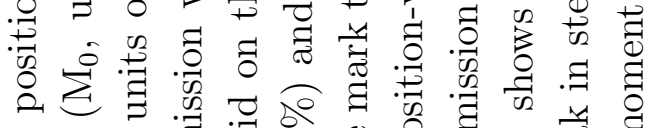

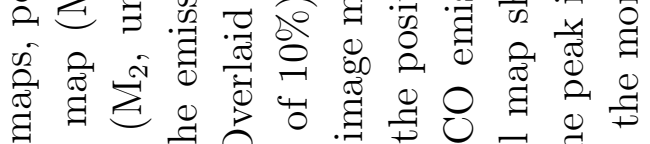

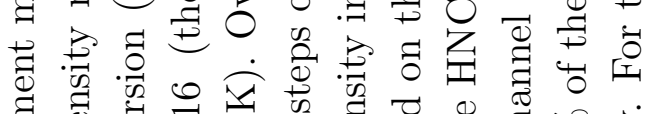

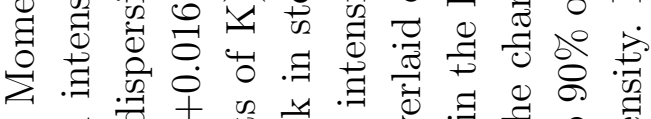

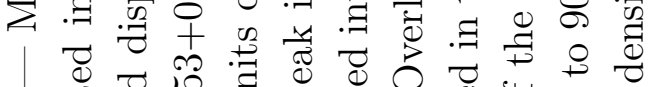
․

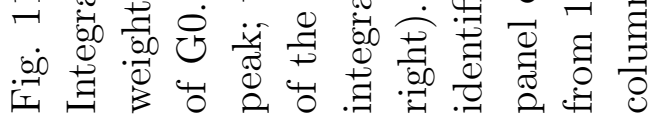

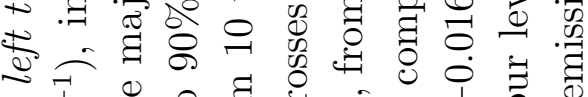



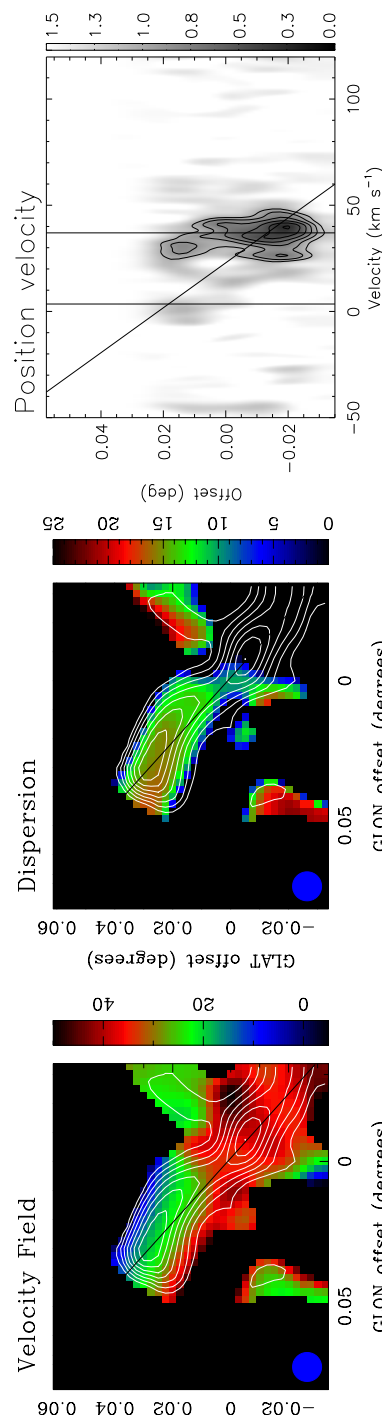

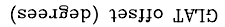

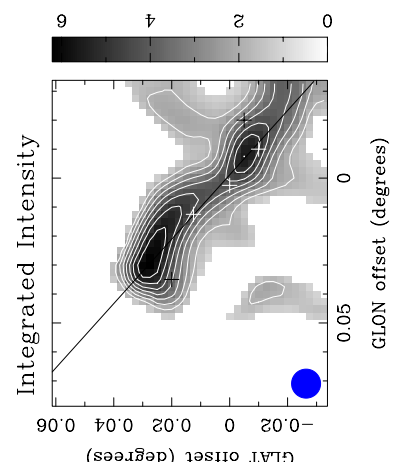

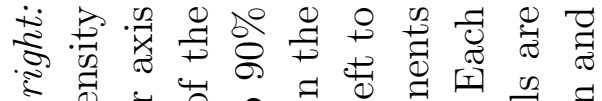

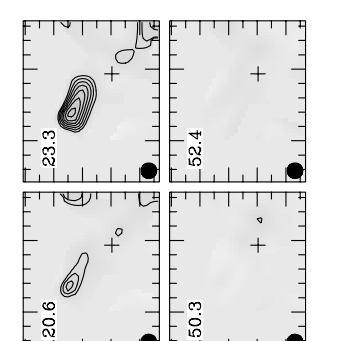

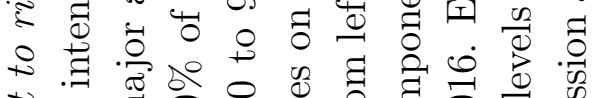

离

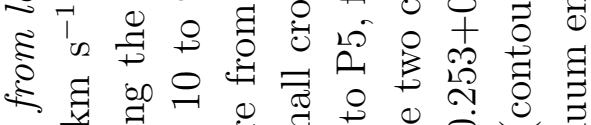

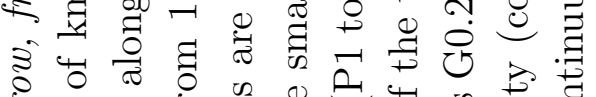

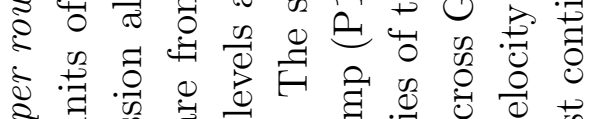

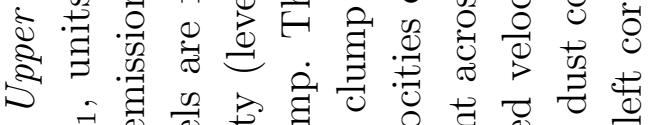

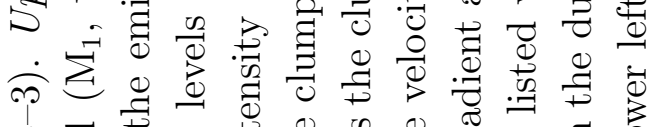
せ

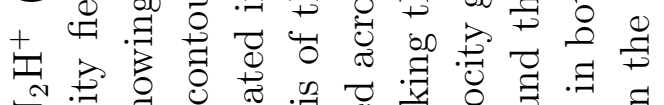

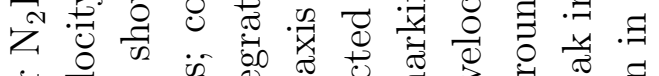
馬 足

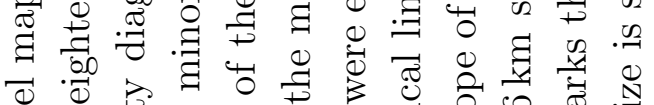

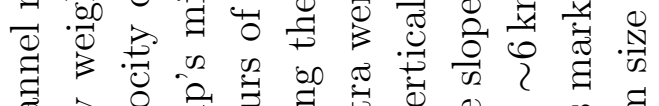

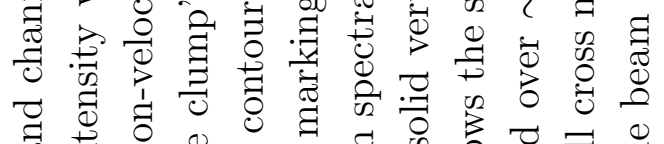

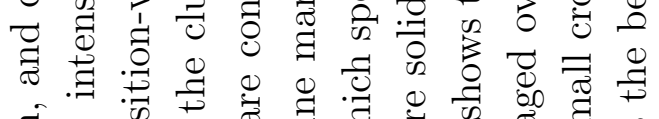

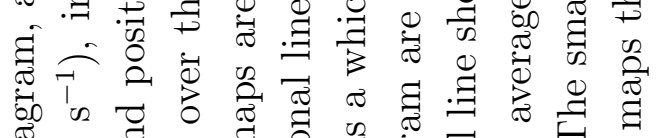

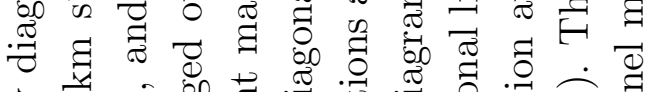

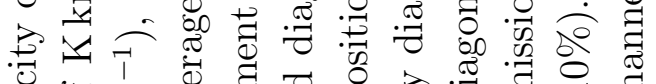
荷

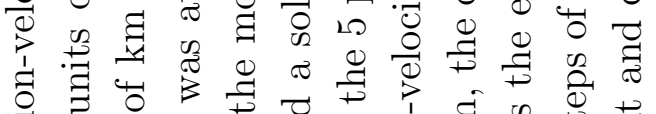

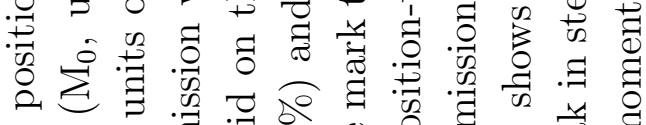
के

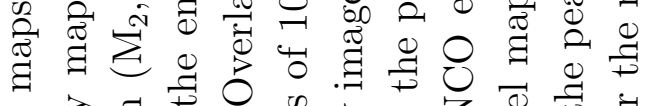

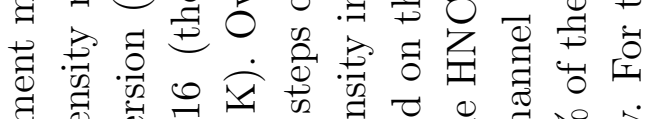

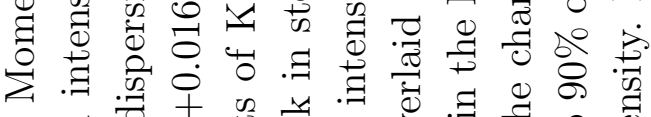

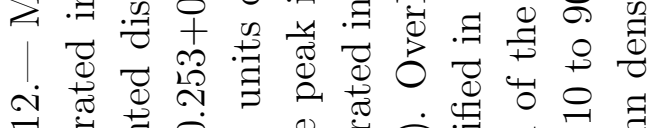

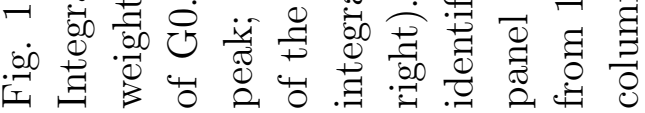



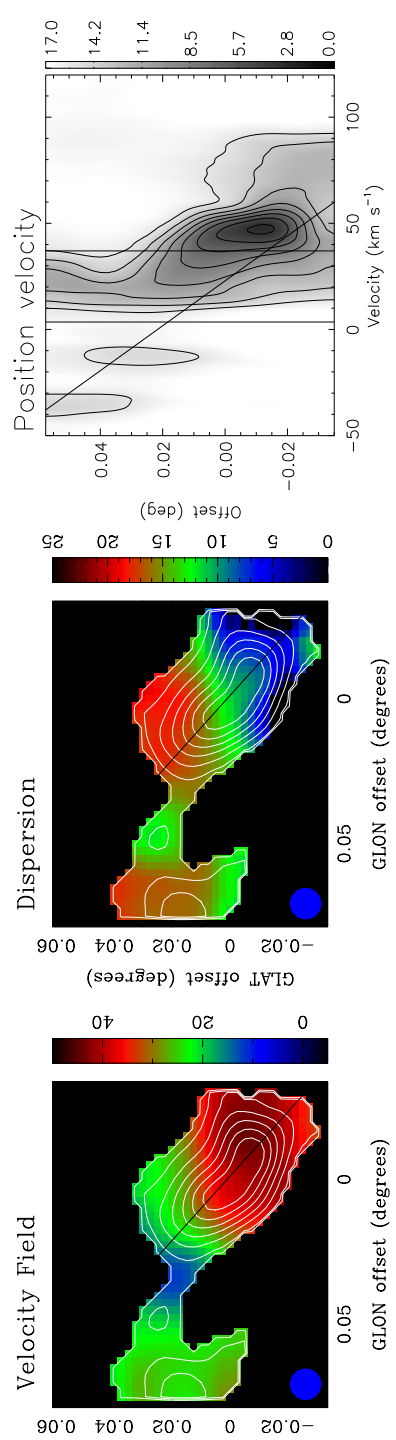

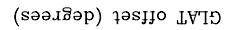

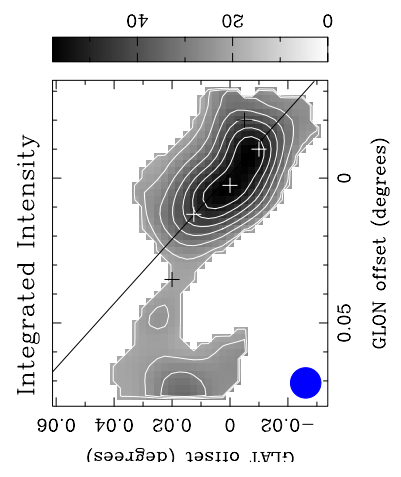

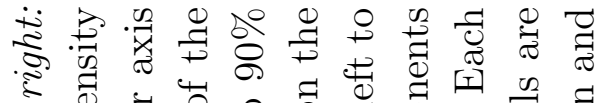

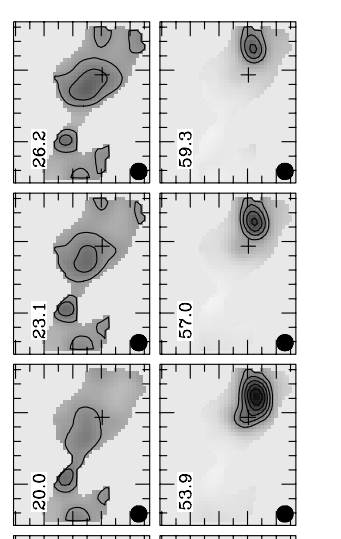

○.

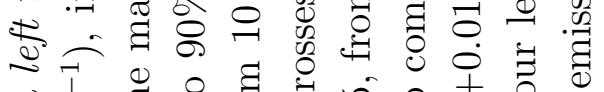

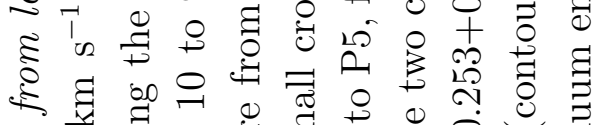

३ฺप्र

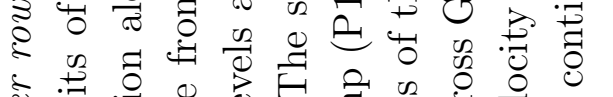

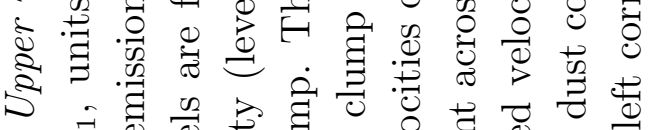

S

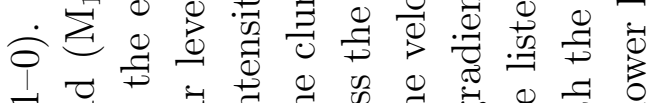

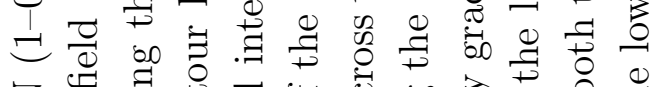

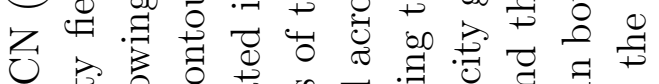

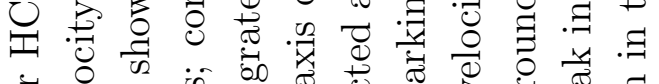

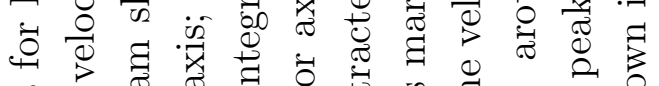

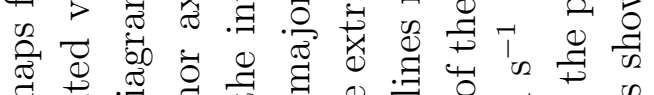

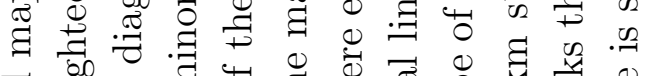

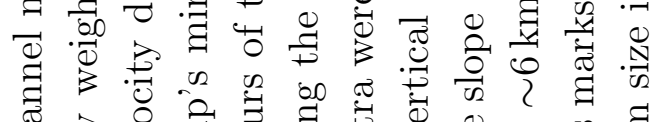

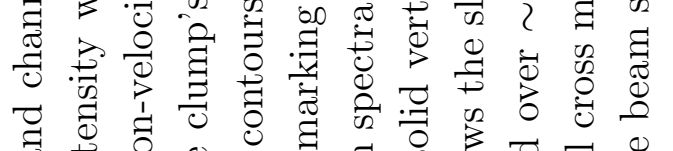

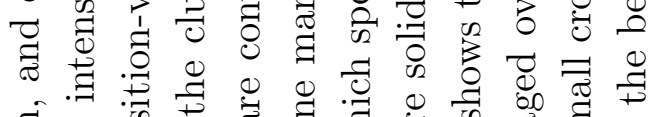

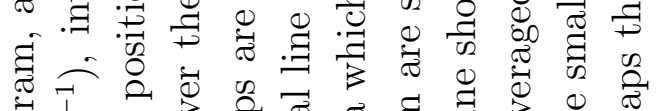

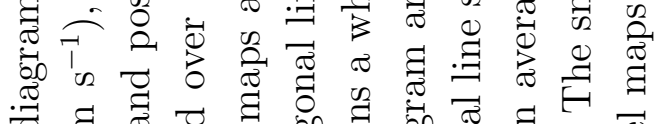

胥

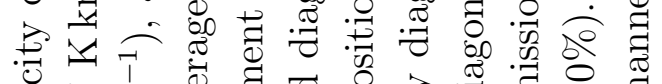

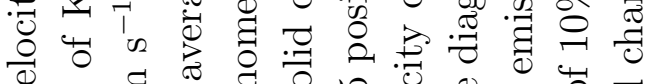

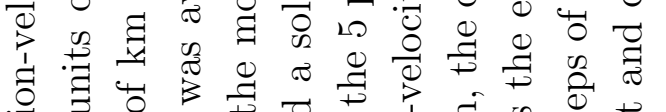

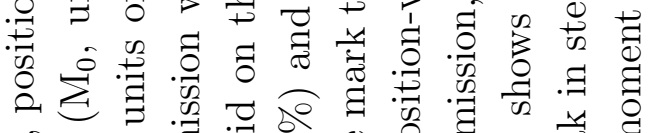

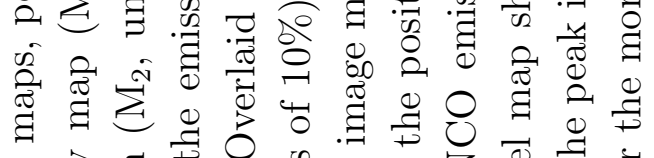

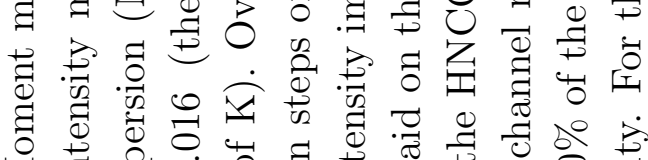

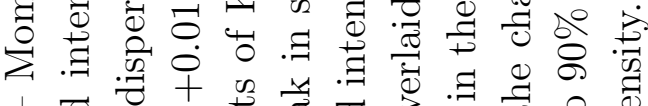

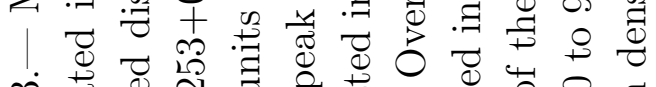

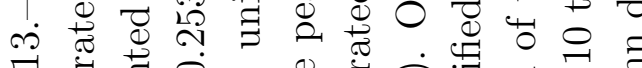

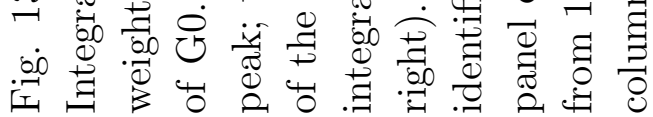



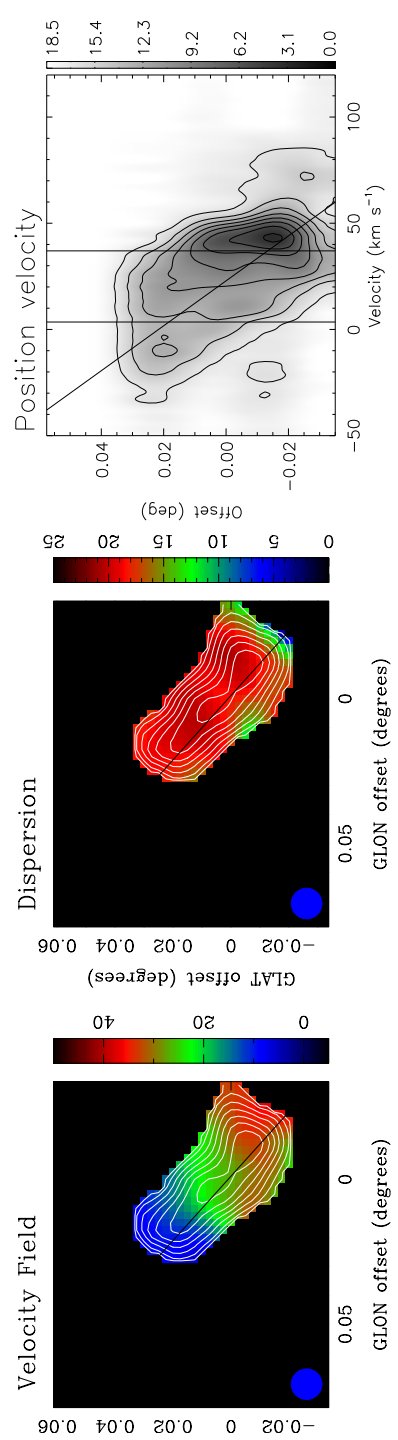

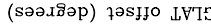
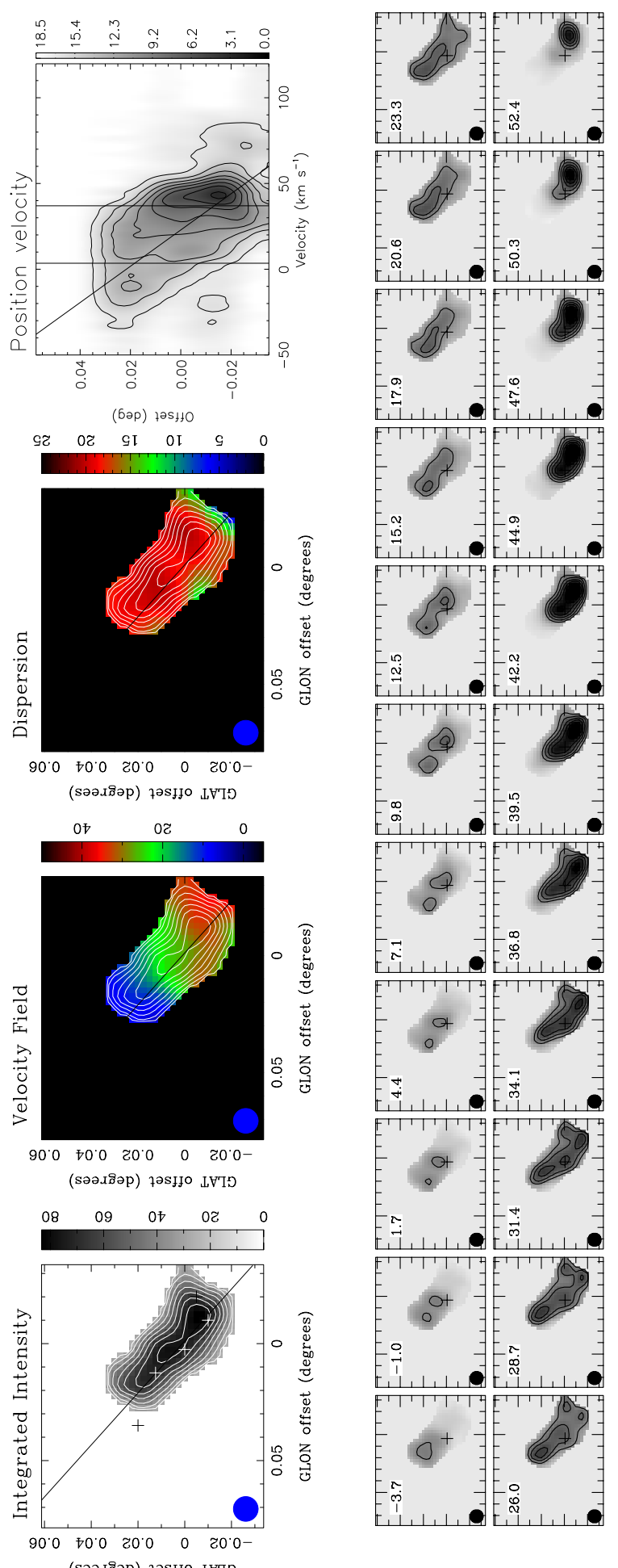

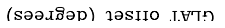

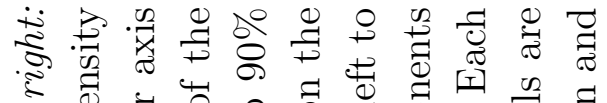

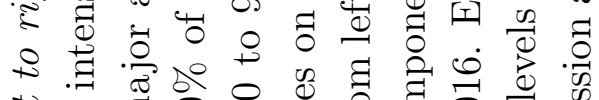

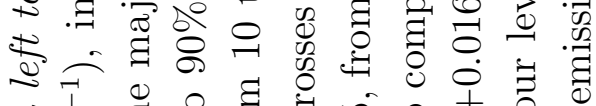

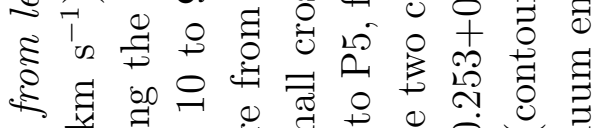

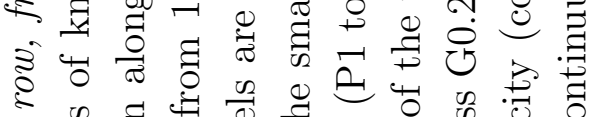

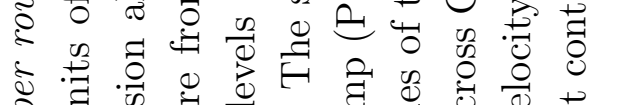

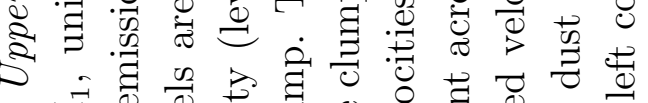

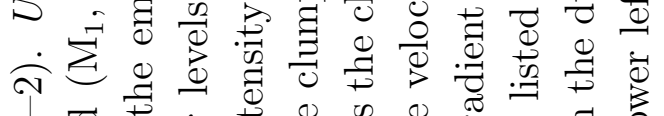

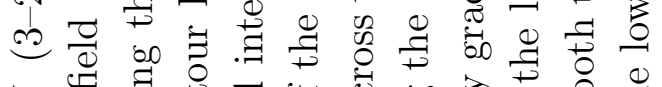

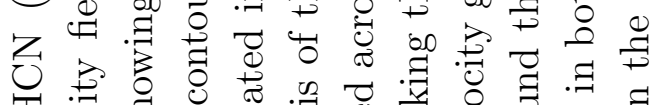

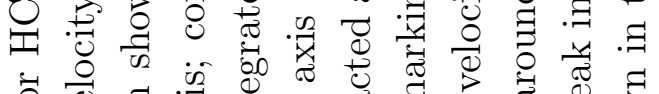

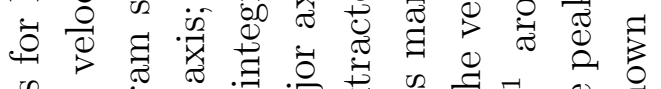

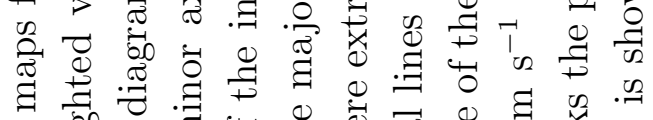

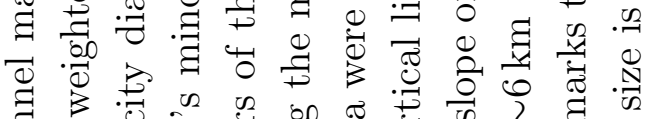

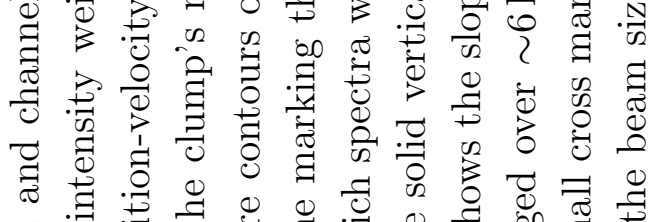

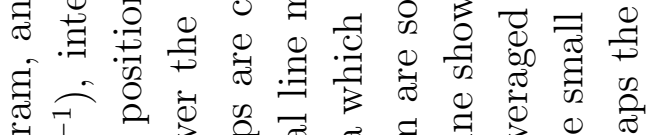

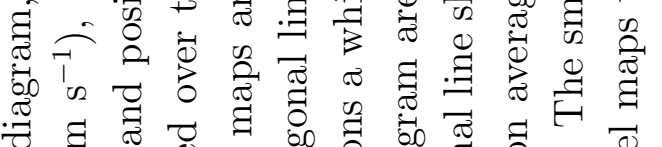

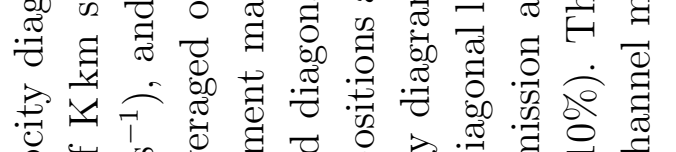

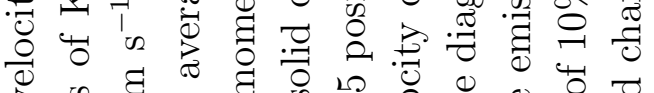

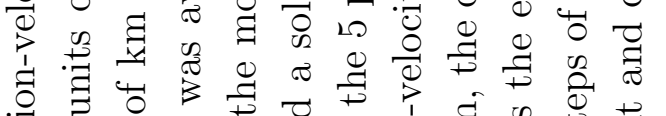

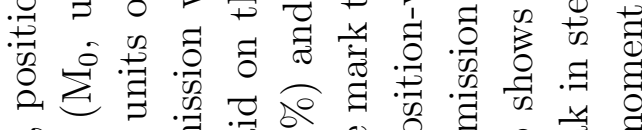

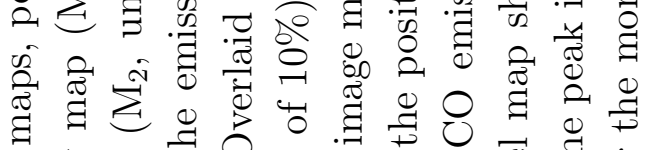

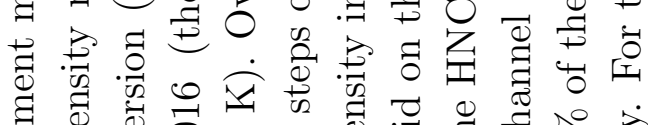

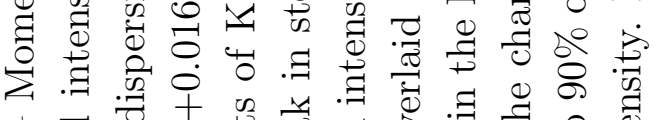

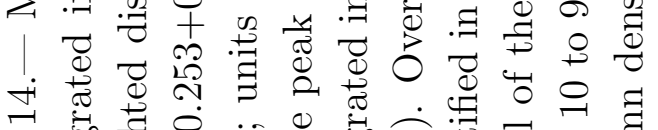

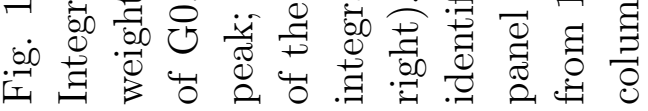



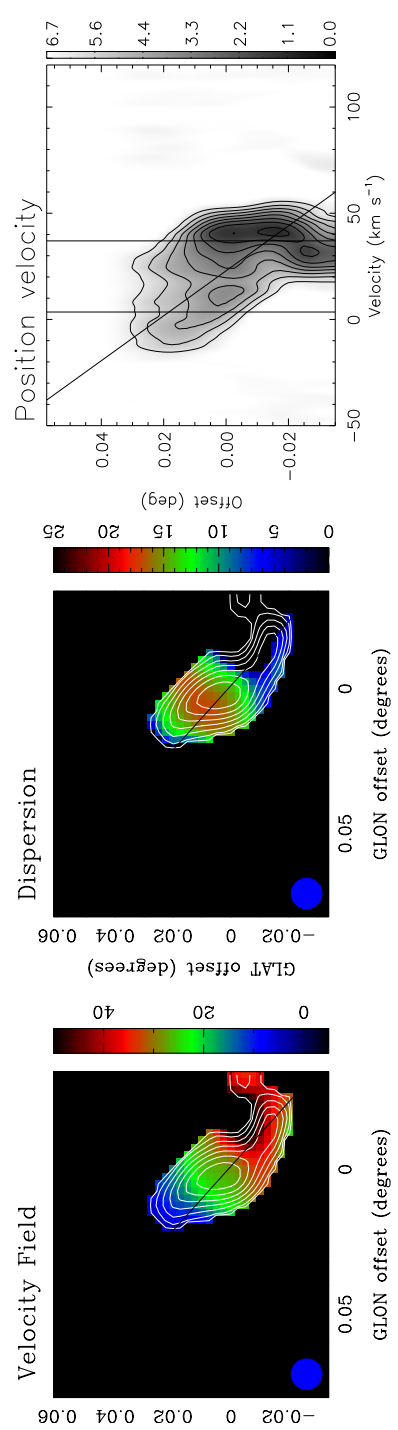

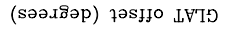

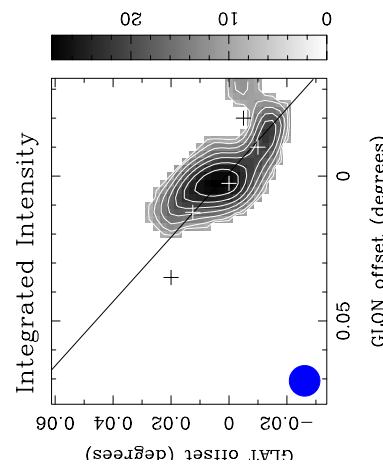

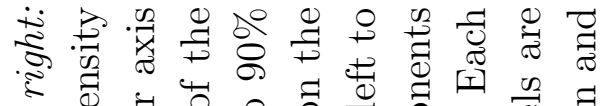
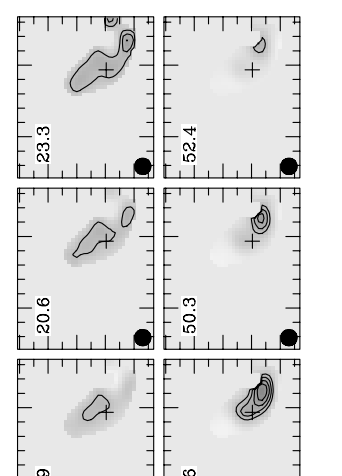

$=\stackrel{8}{-2}$
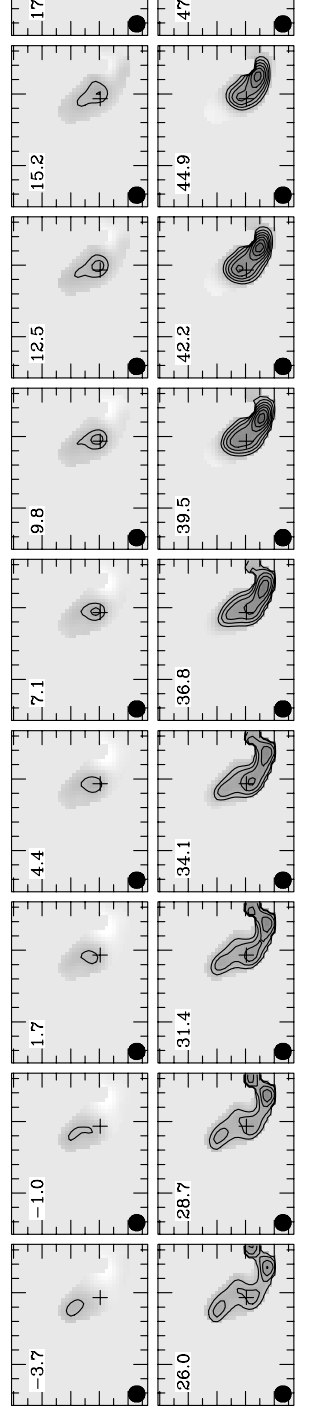

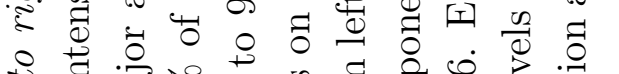

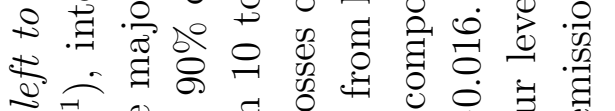

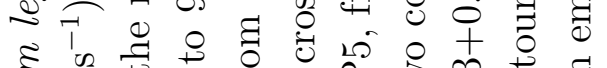

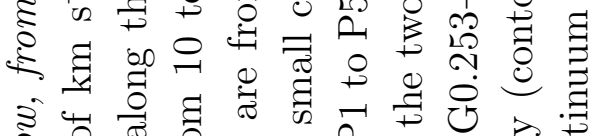

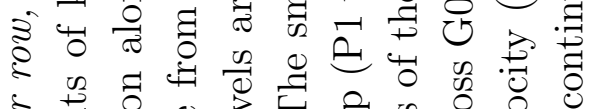

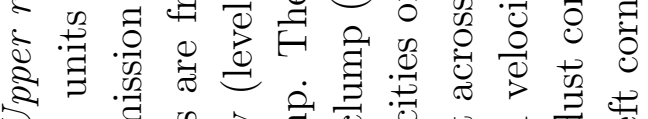

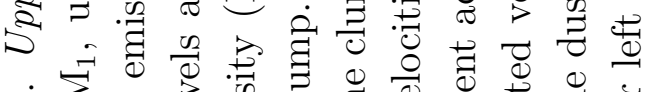

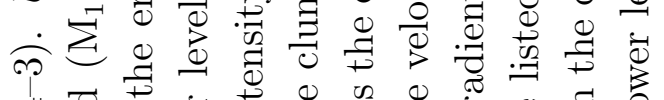

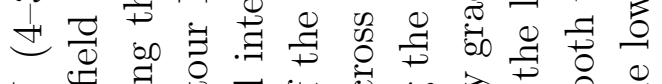

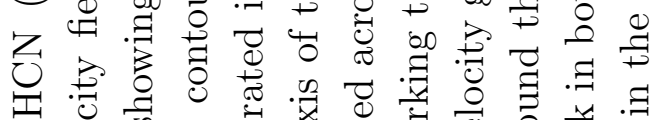
- 范

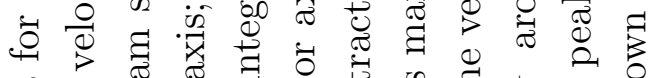
蛋

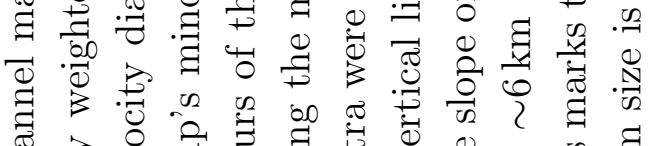

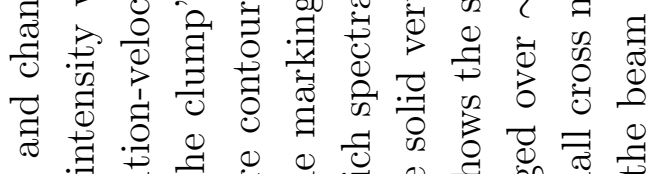

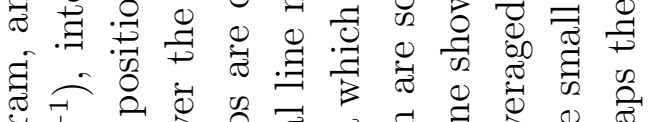

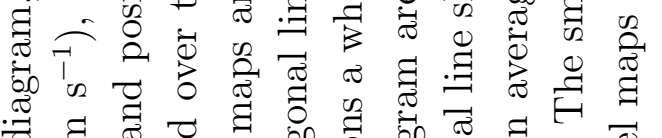

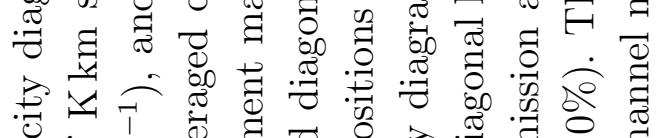
范

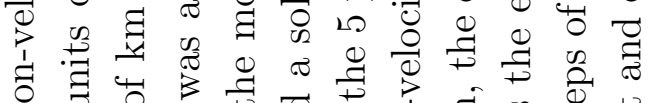

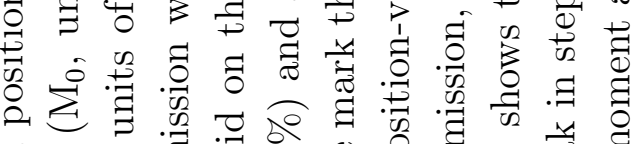

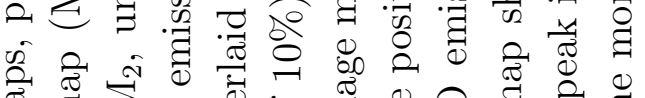

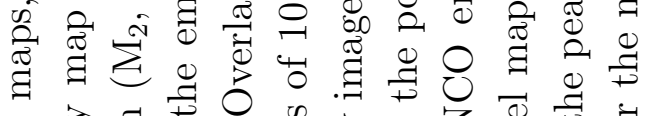

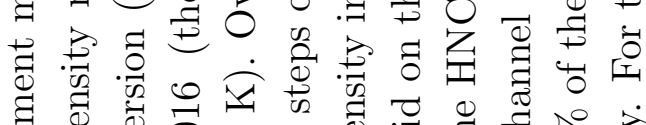

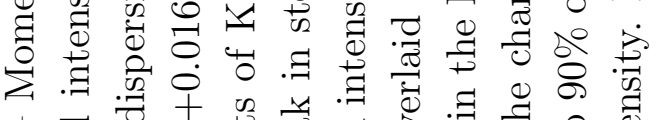

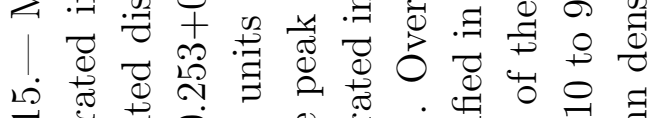

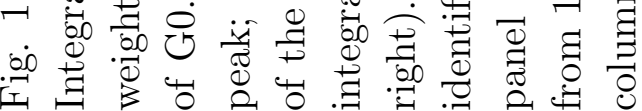



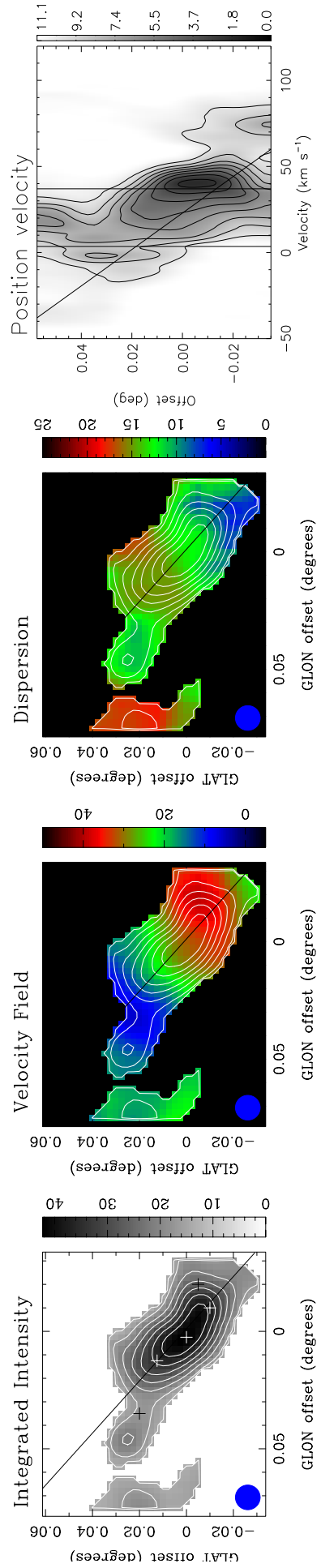

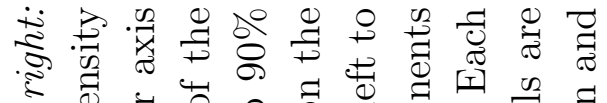

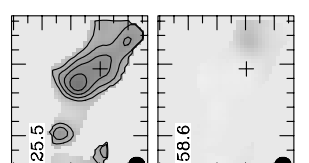

○ 岕 ह i

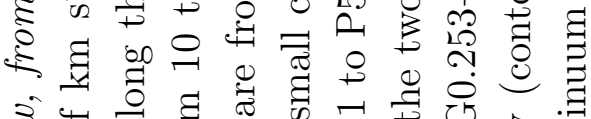

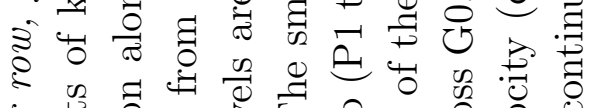
㐫. 节.

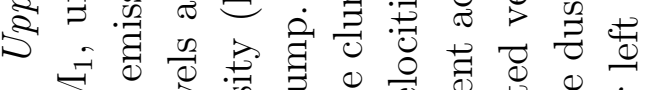

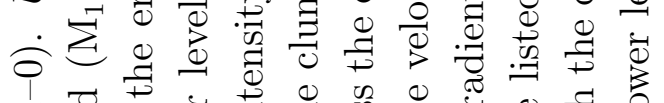

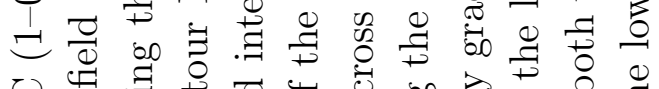
乙山.

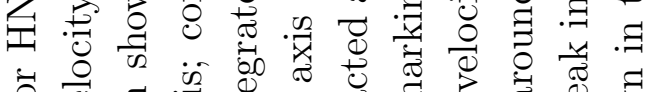
苛 苛

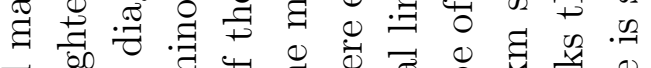

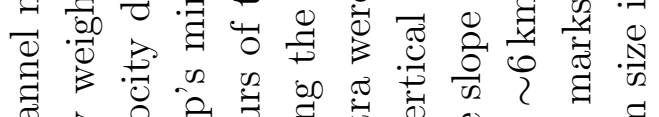

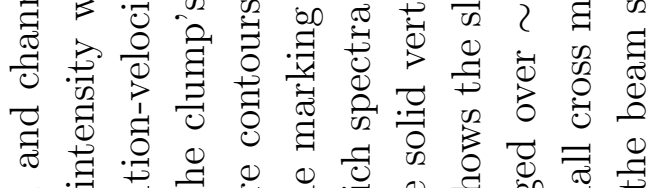

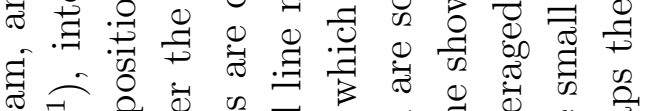

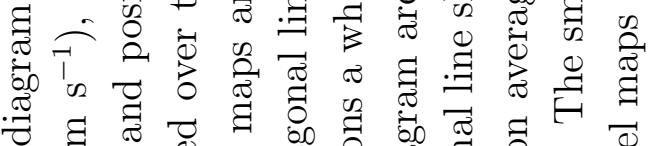

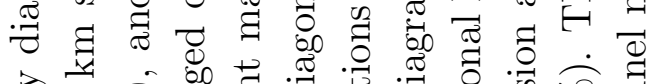
焉

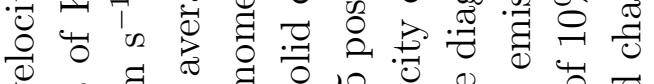

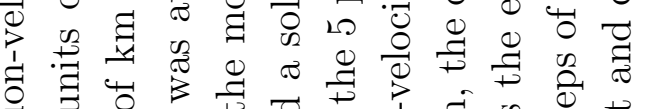

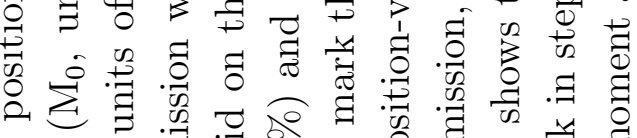

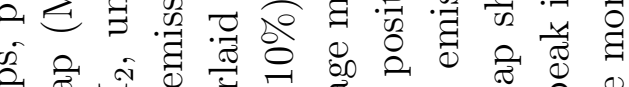

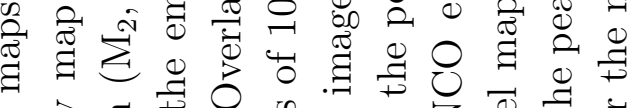

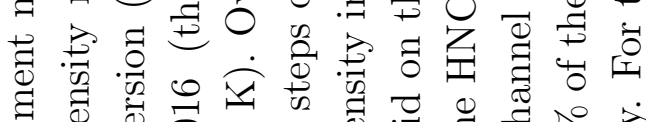

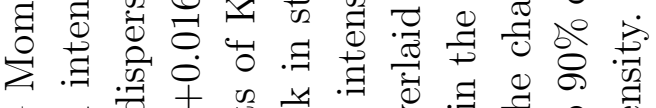
0 赵

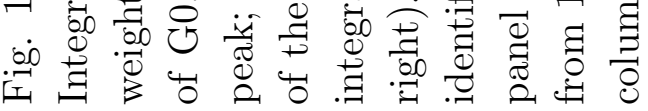



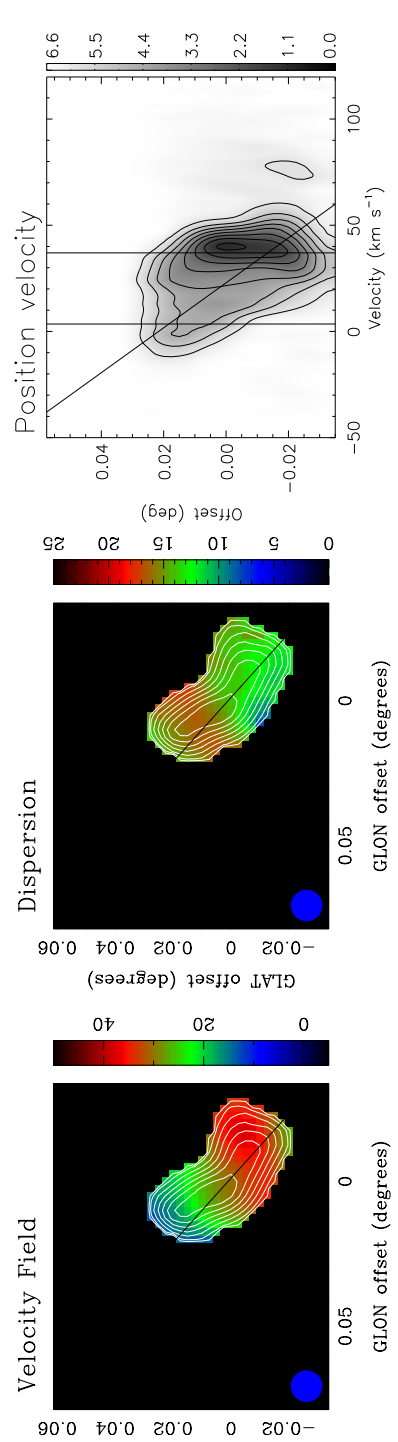

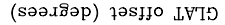

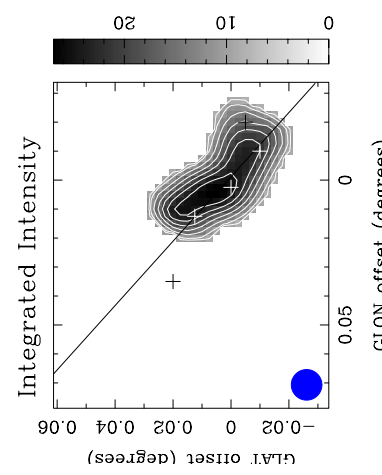

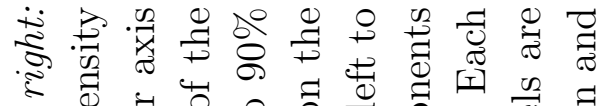
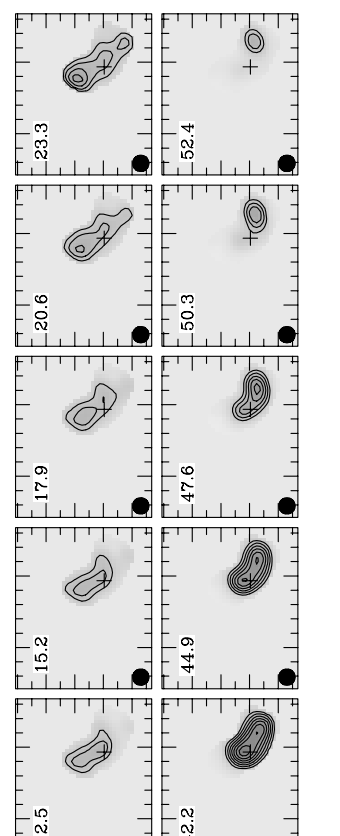

$=$
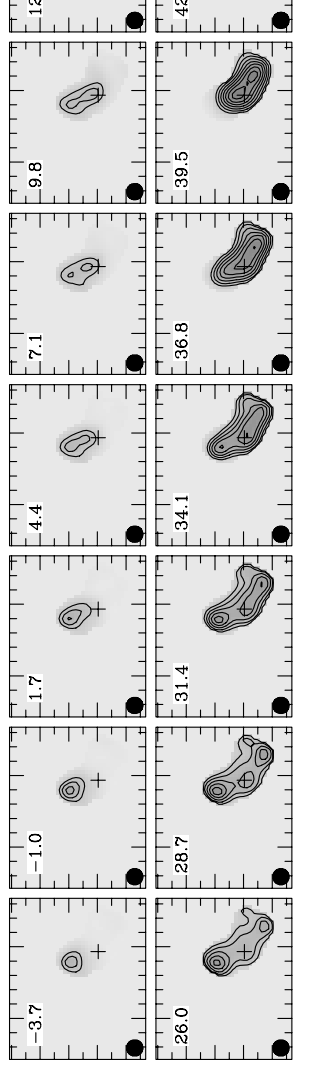

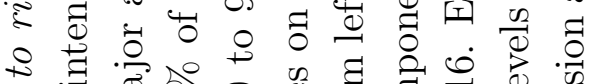
芯 है।

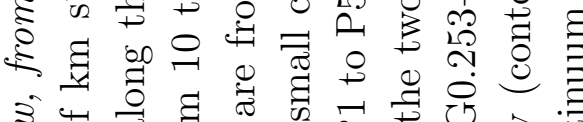

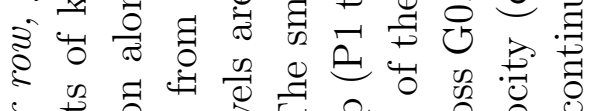
¿.

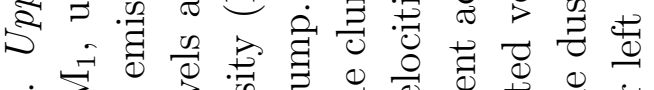

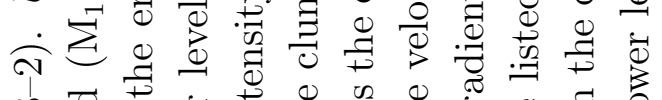
ब. 乙 留

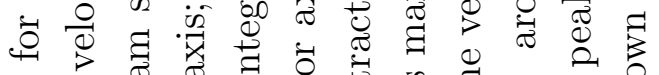
足

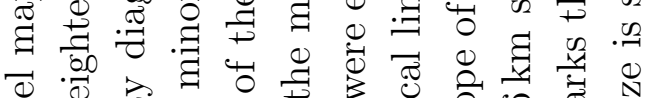

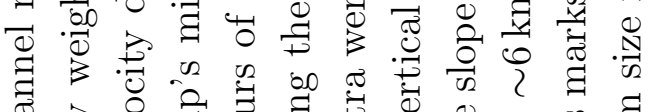

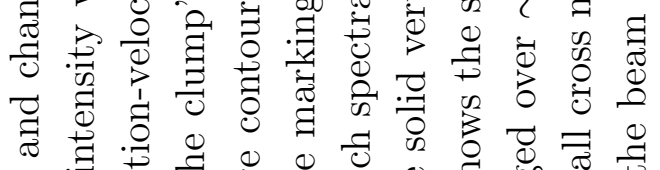

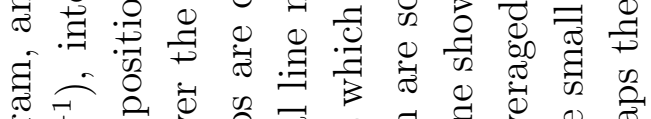

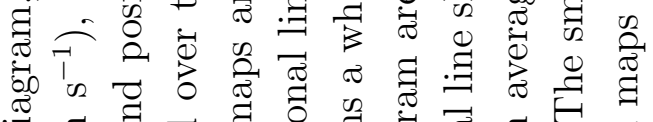

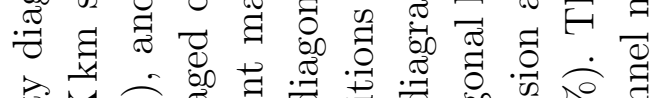
焉

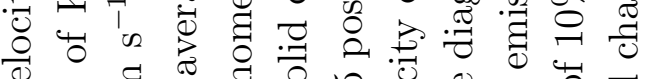

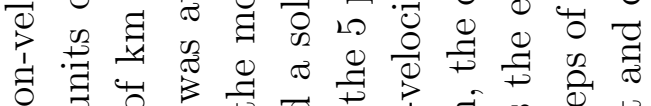

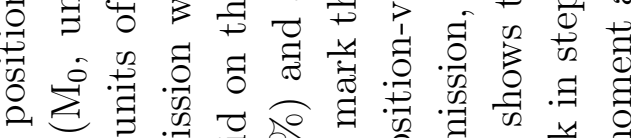

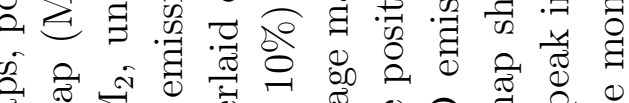

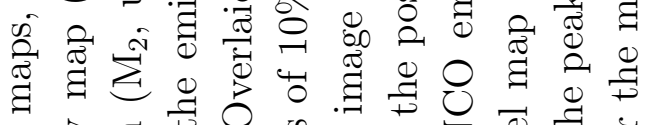

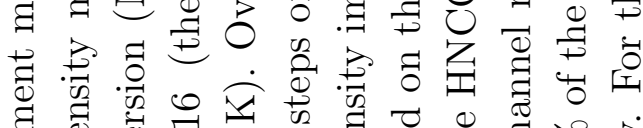

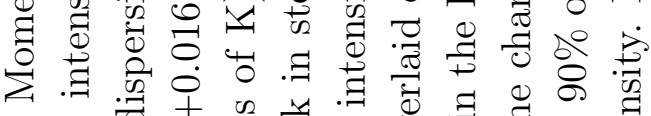

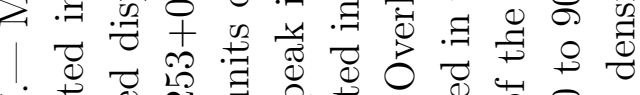
․ 屯

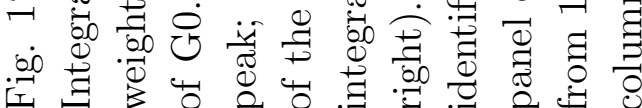



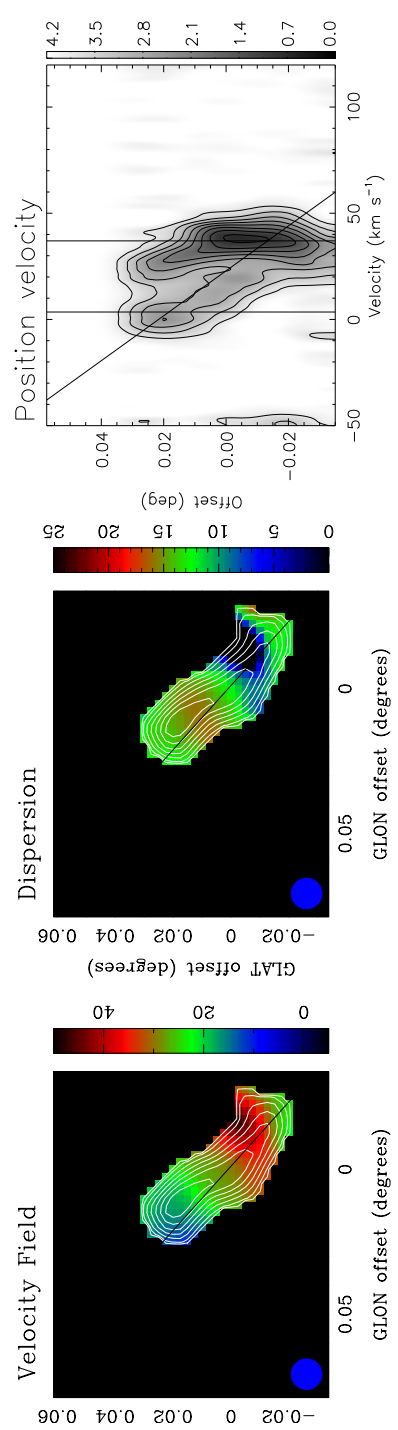

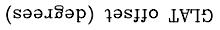
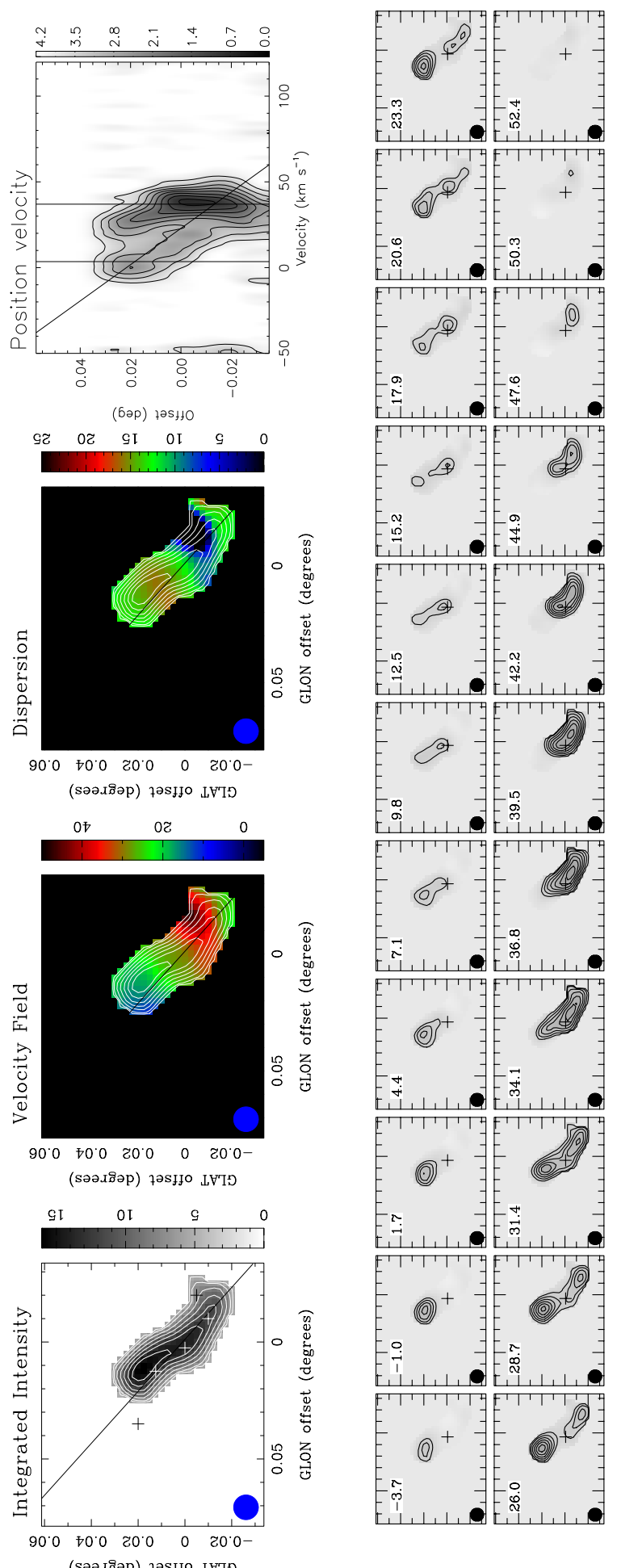

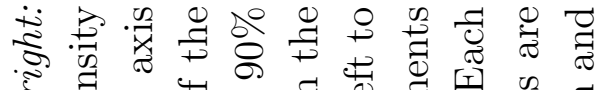

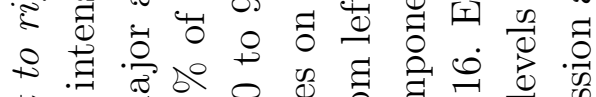

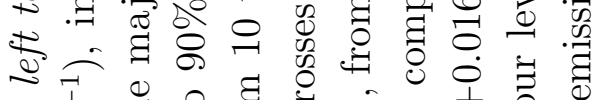

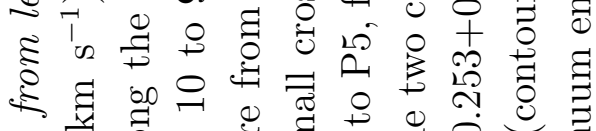

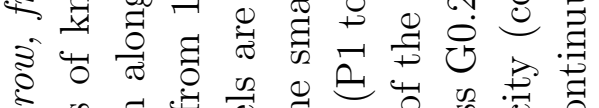

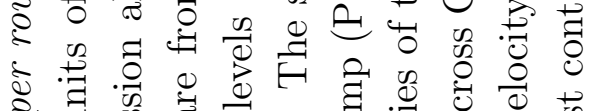

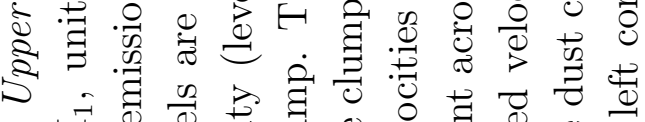

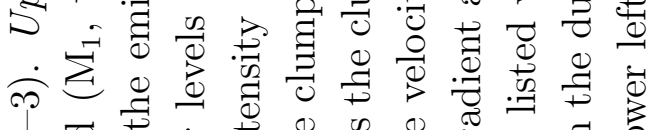

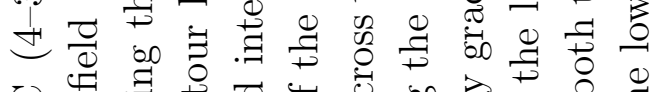

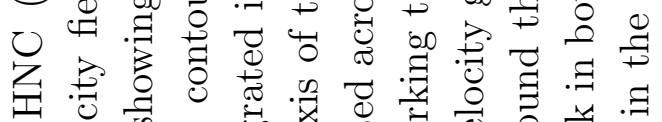

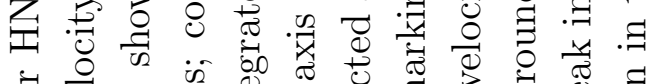

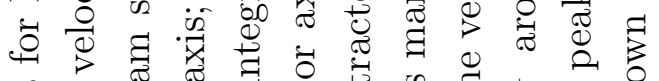

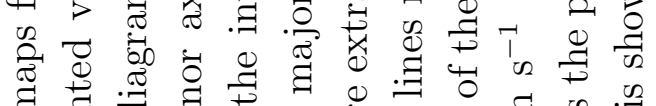

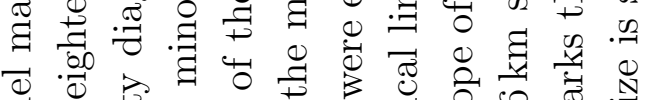

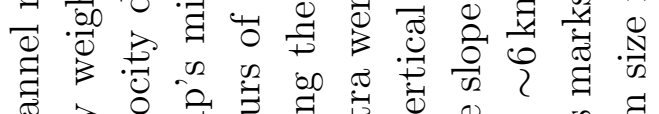

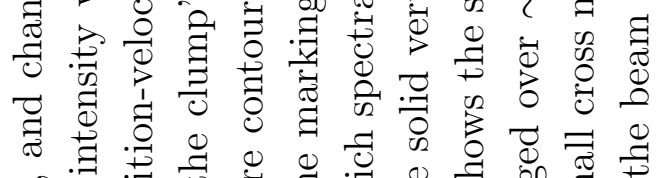

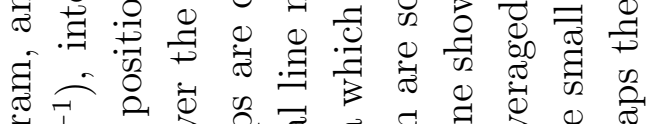

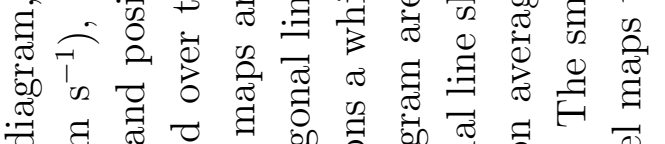

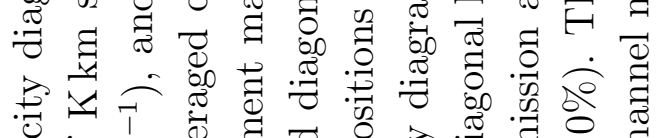

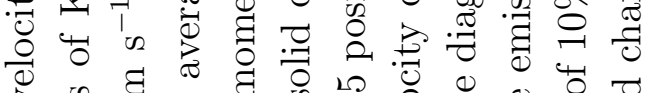

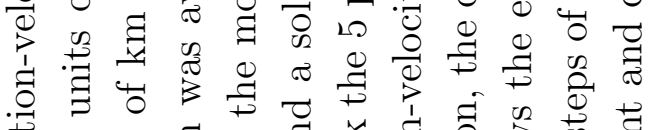

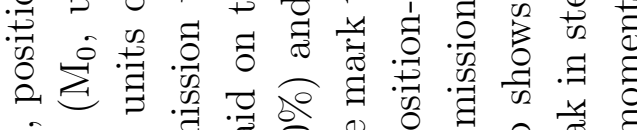

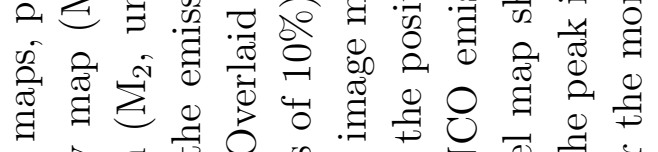

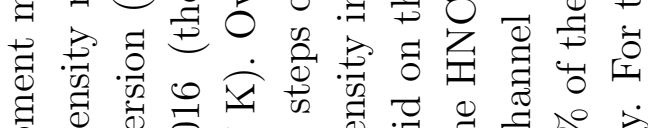

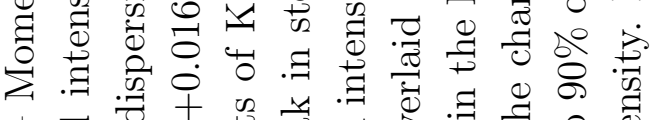

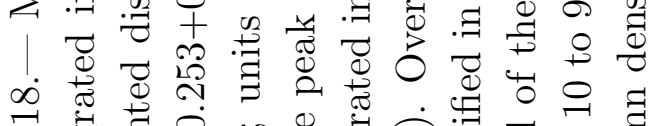

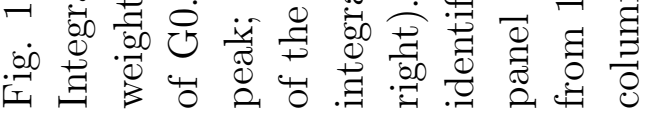



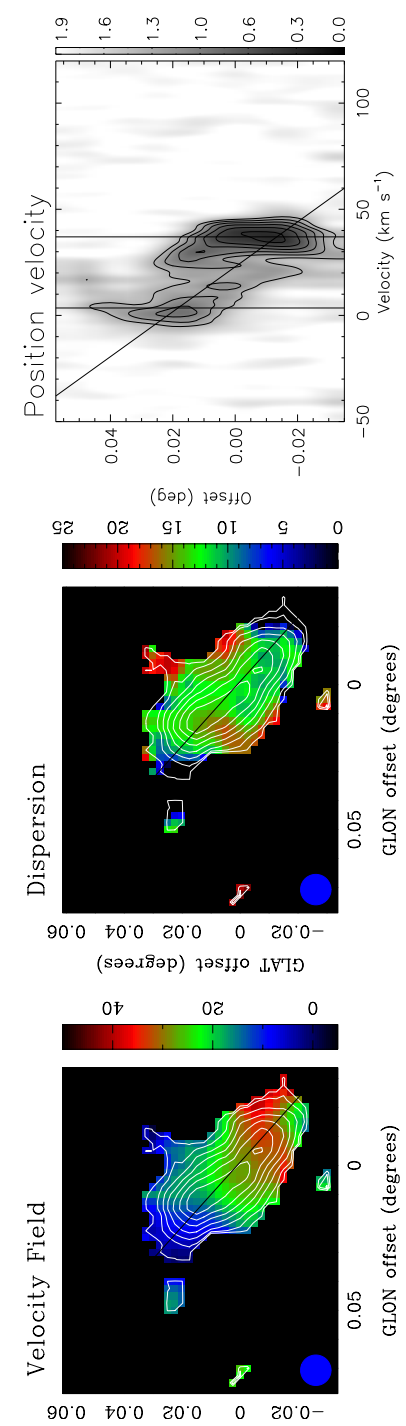

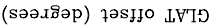

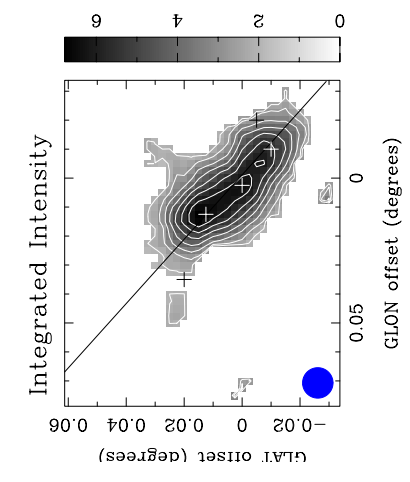

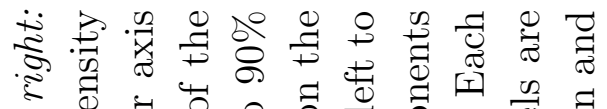

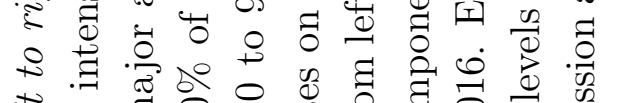

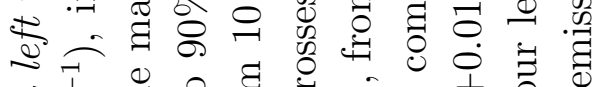

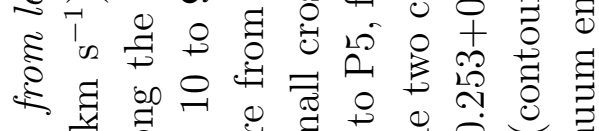

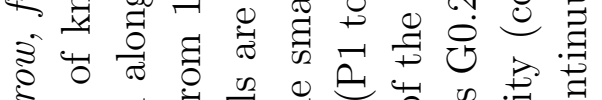

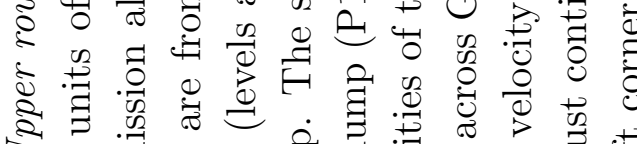

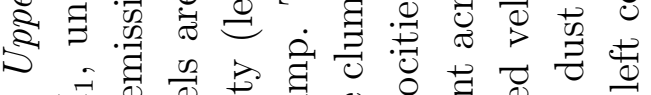

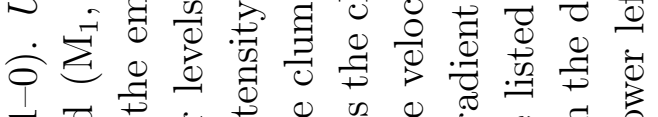

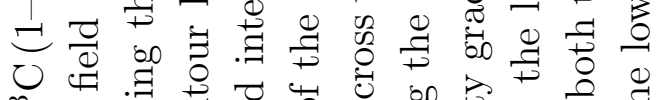

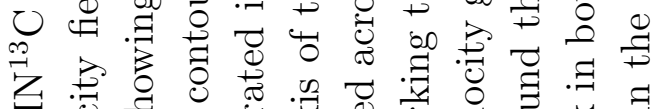

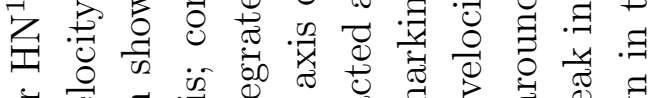

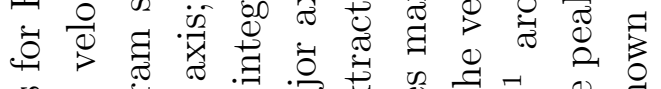

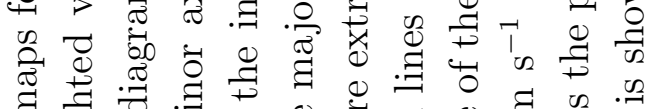
晋

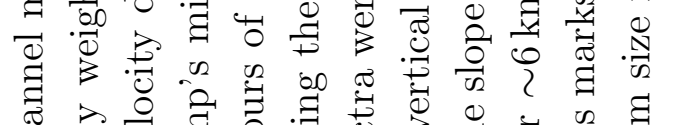

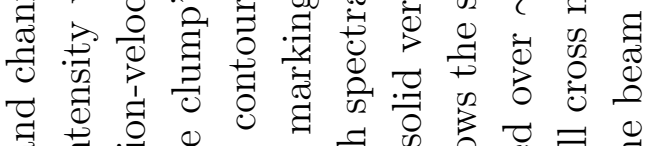

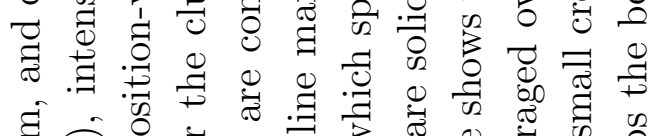

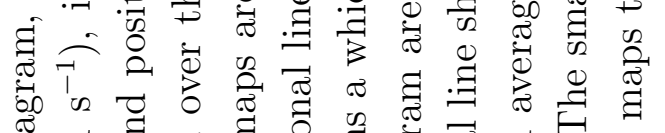

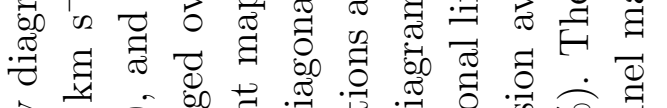

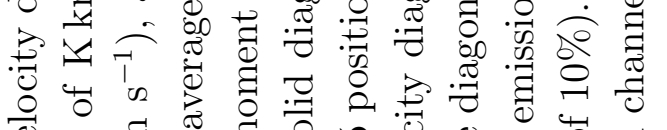

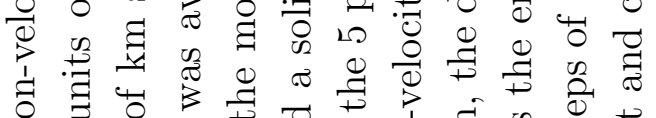

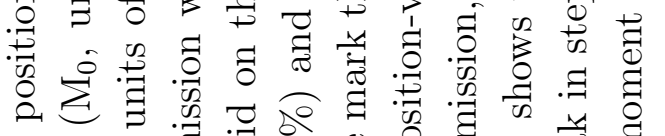

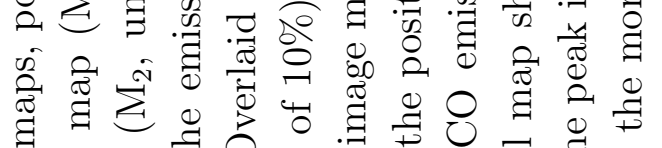

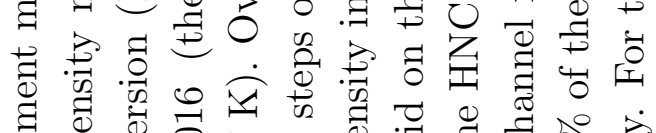
gु

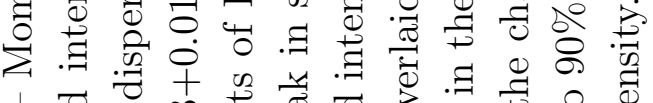

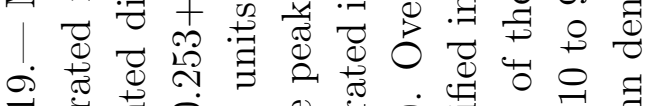

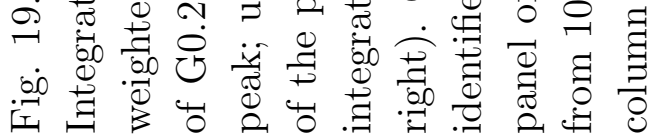



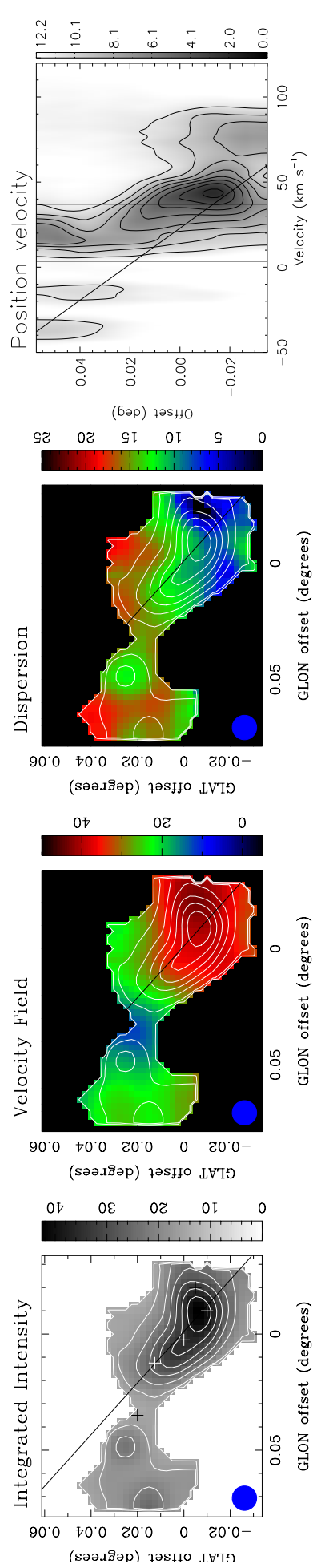

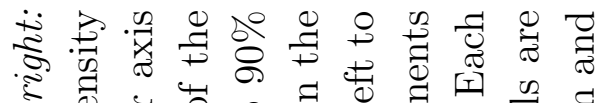
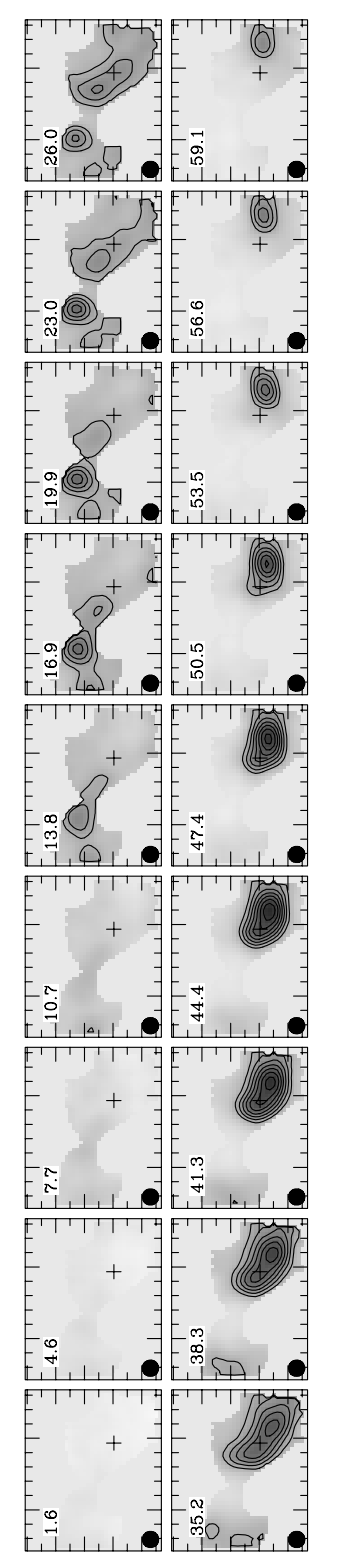

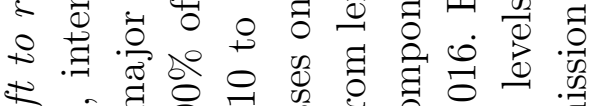

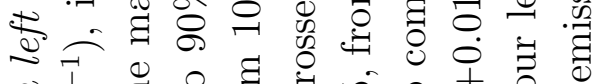

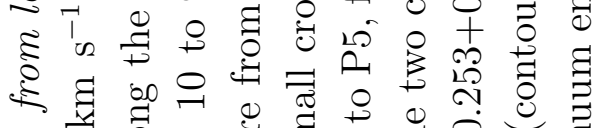

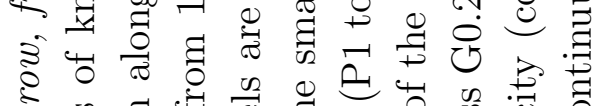

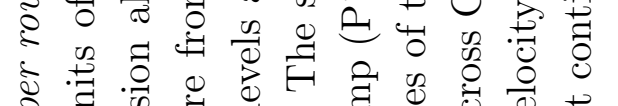
离苛.

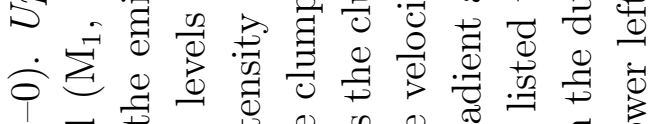

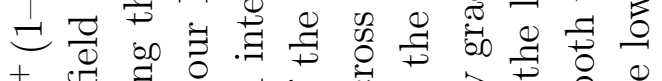

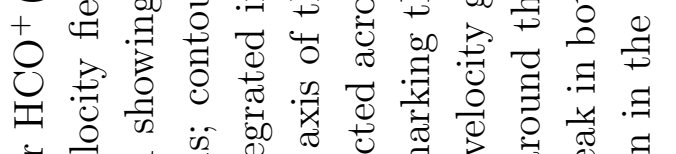
馬

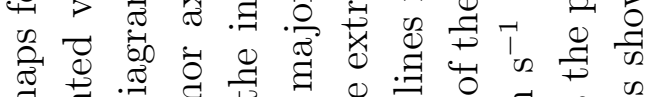

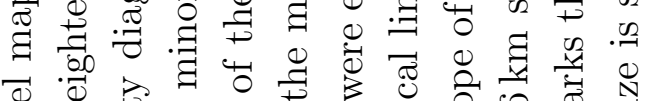

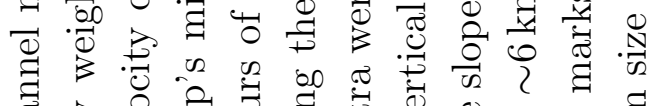

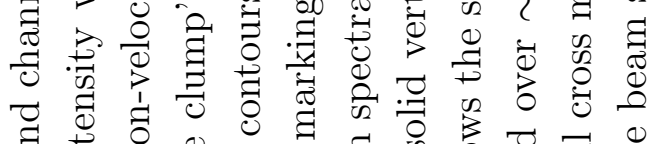

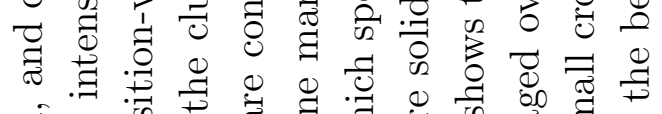

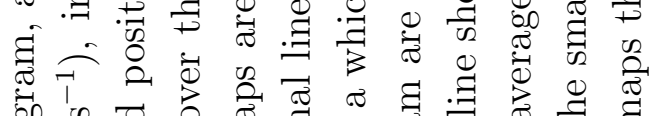

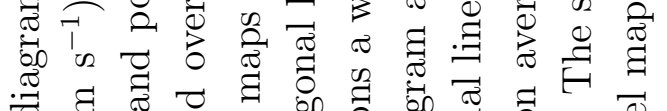
要

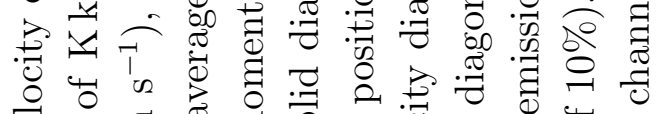
苚

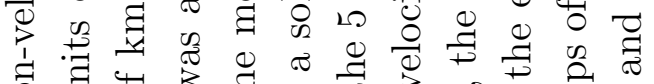

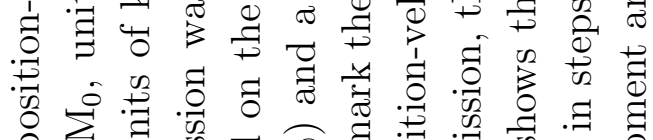

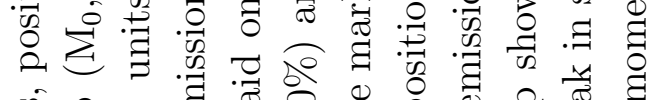

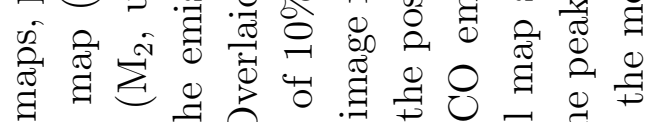

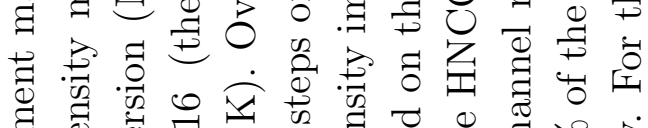

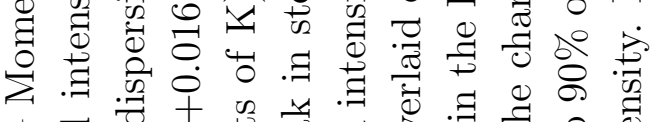

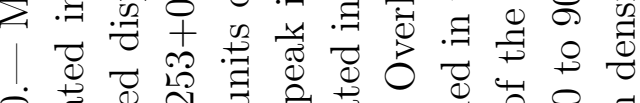
穴芯芯

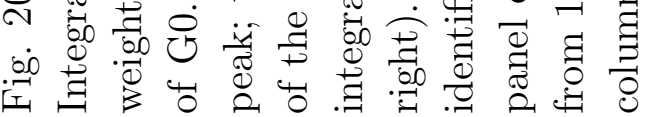



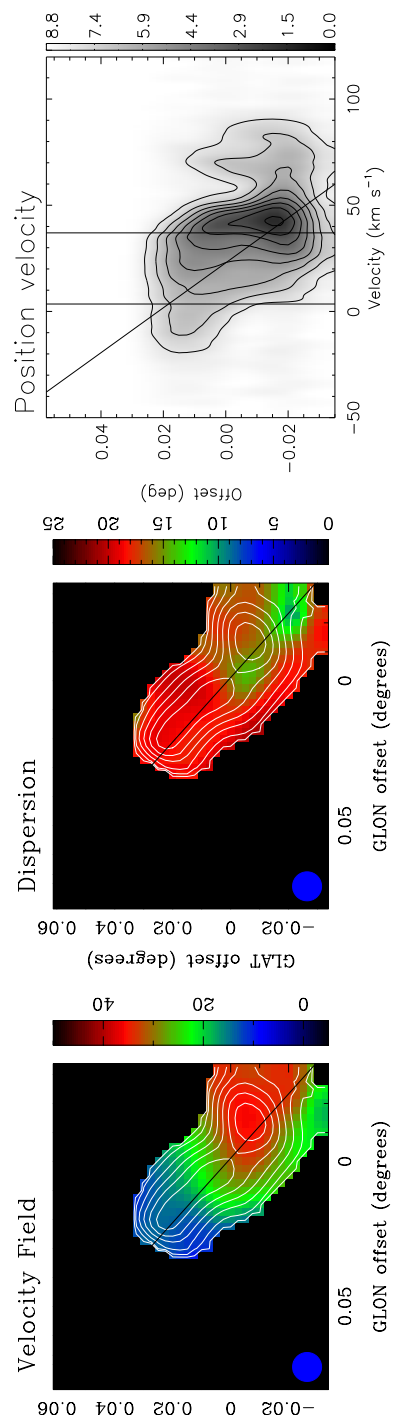

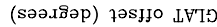

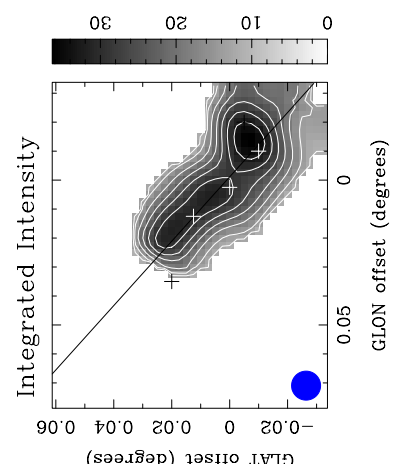

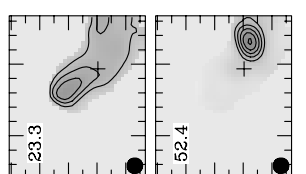
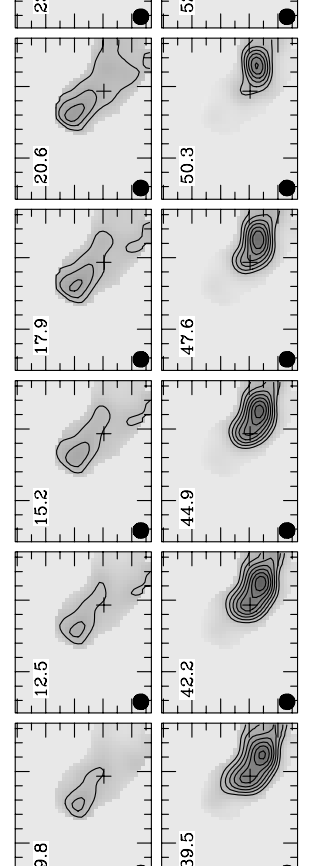

-

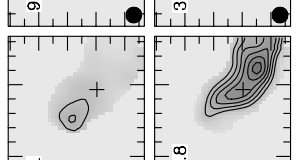

$=$
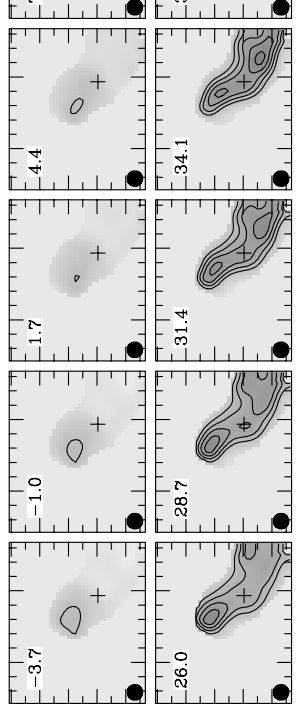

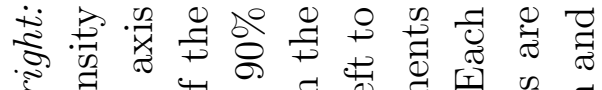

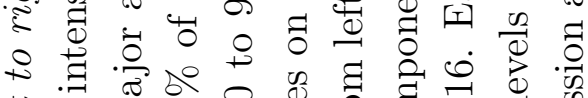
녕 I

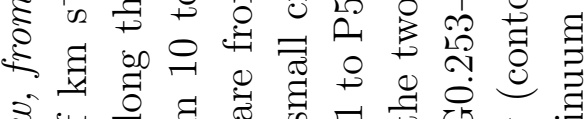

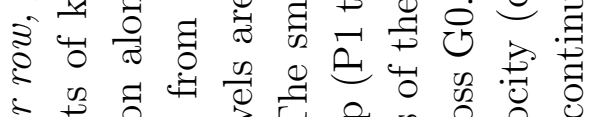

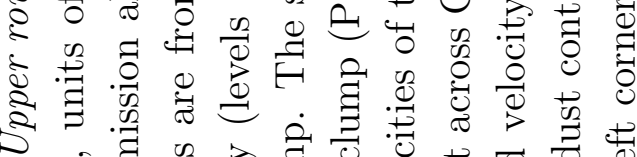

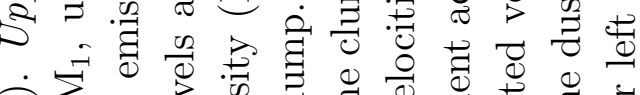

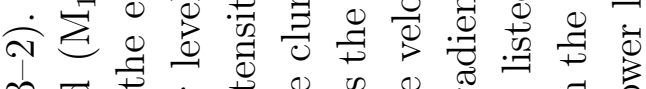

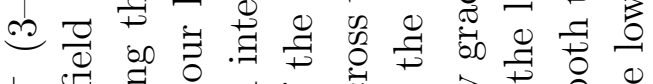

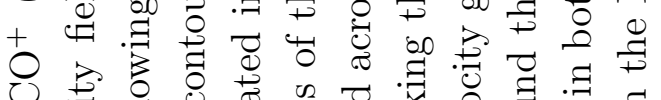

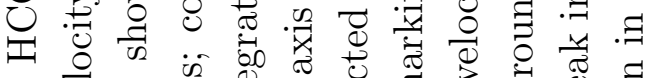

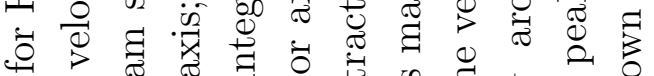

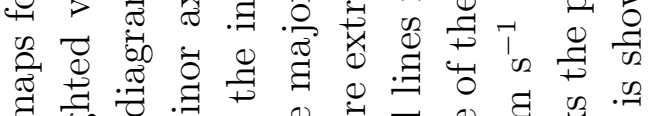

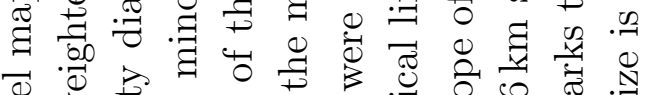

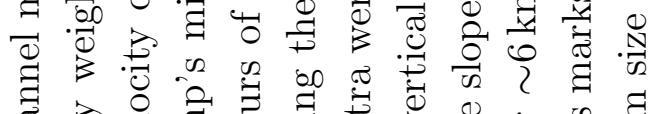

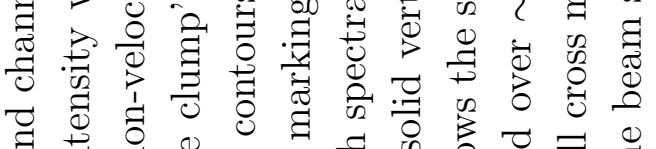

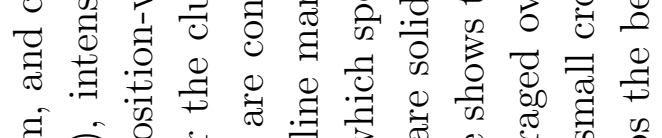

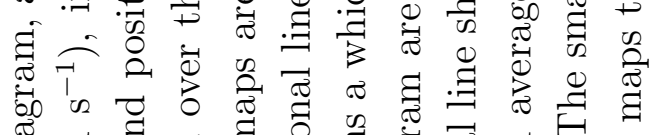

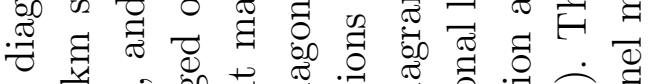

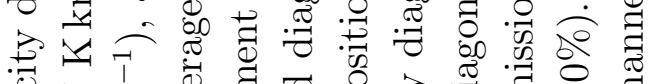

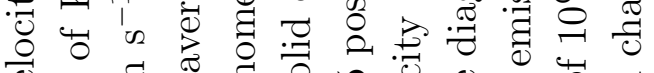

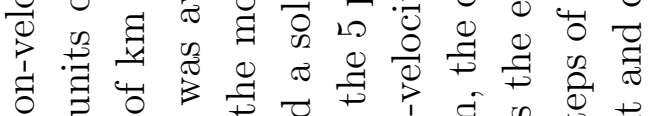

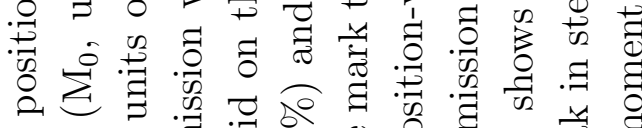

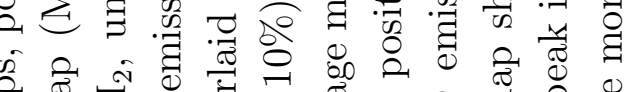

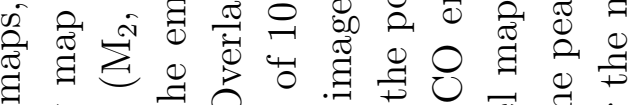

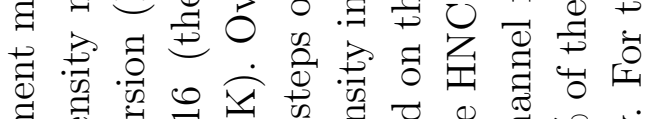

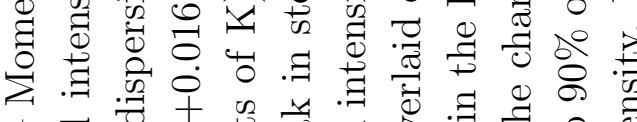

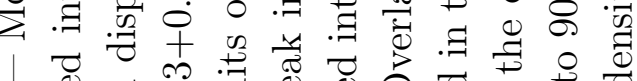

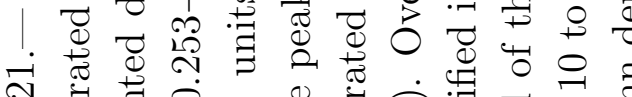

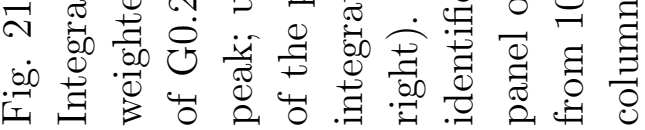



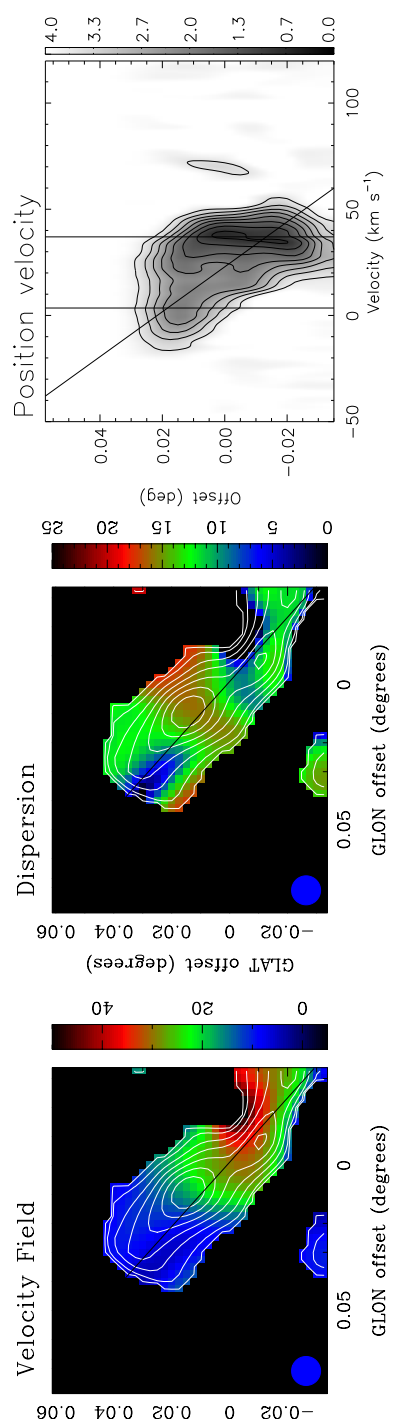

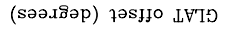

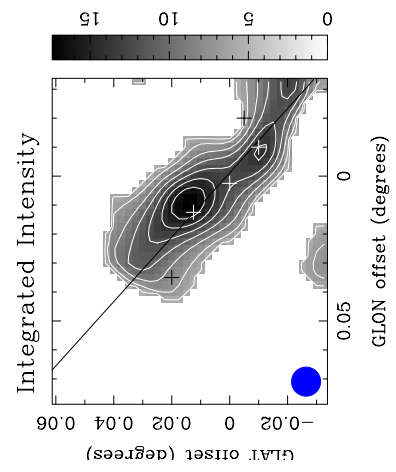

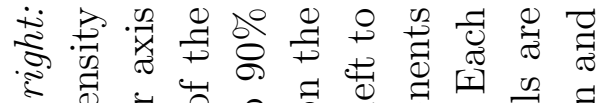
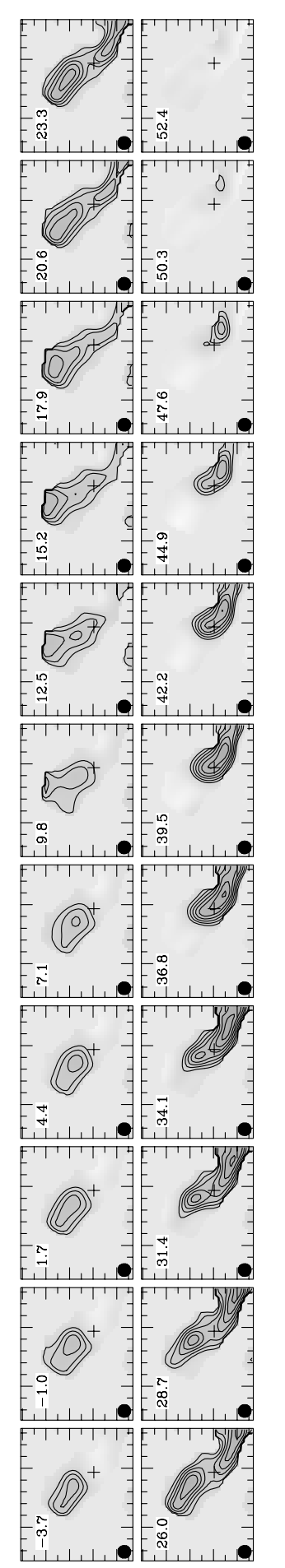

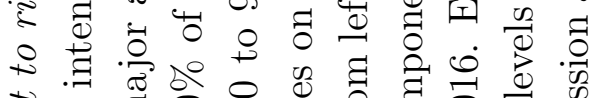


is

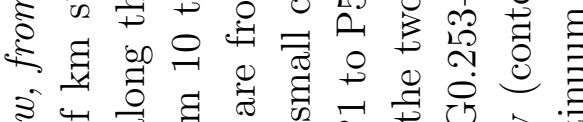

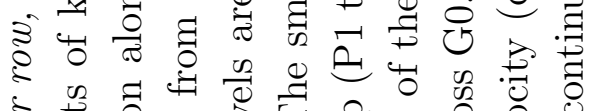

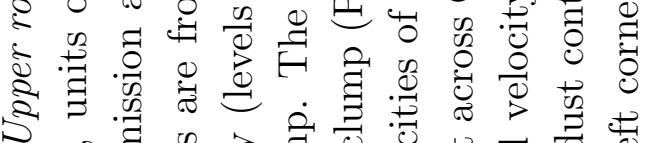

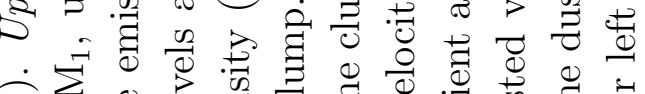
के

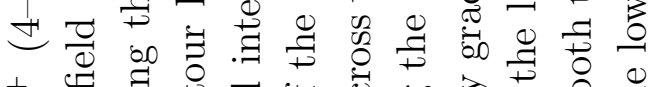

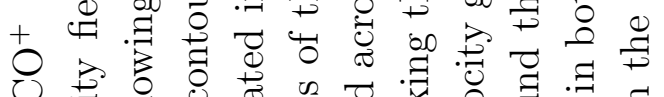

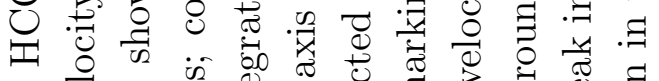

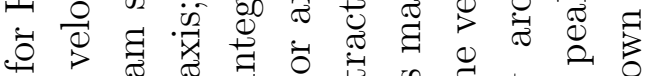

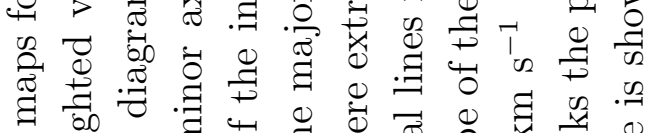

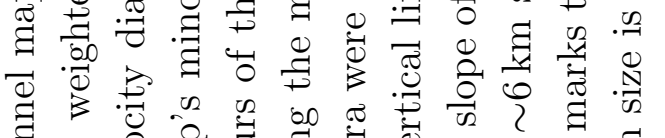

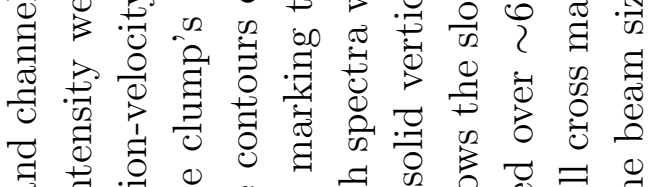

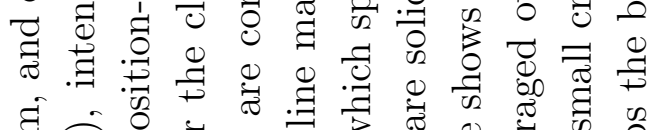

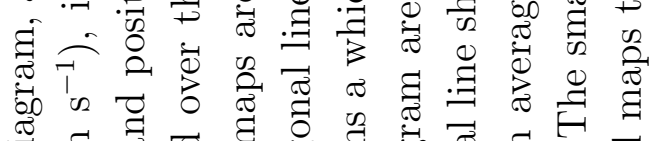

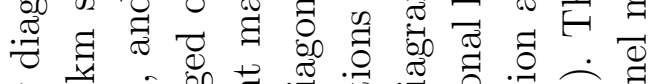

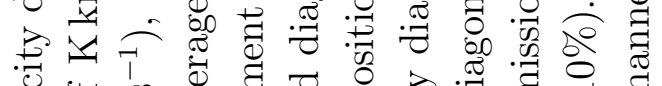

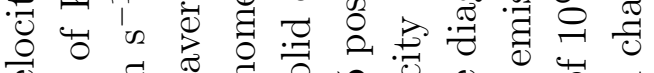

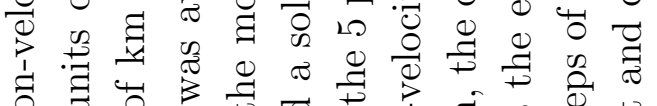

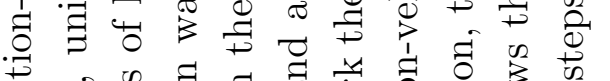

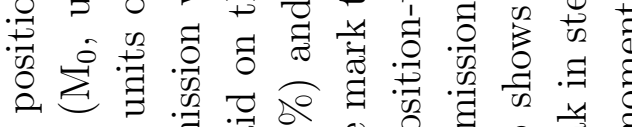

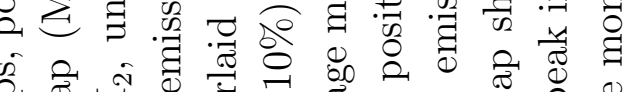

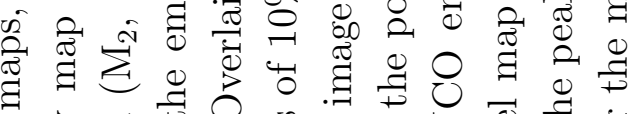

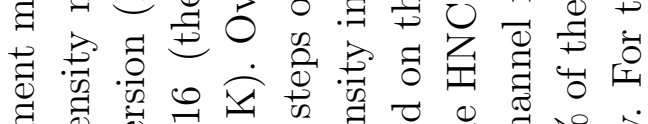



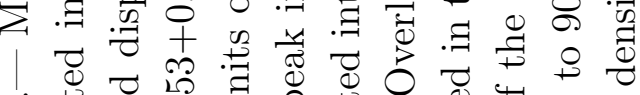
ล่ N 

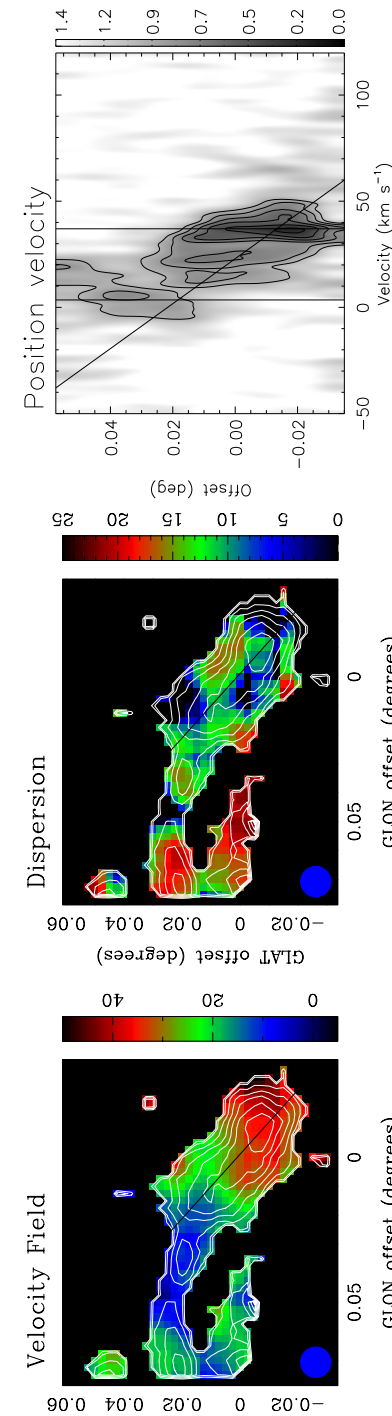

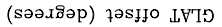

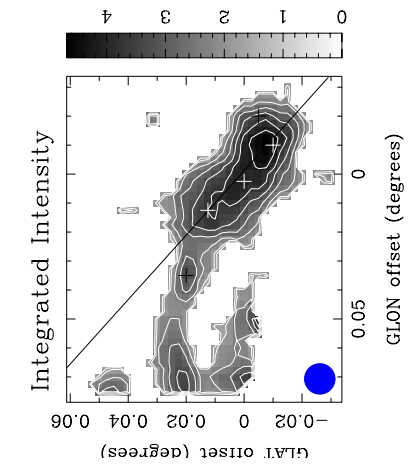

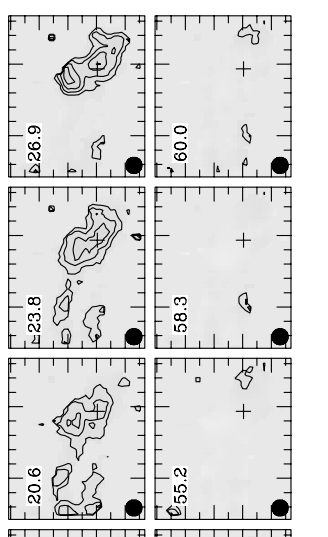
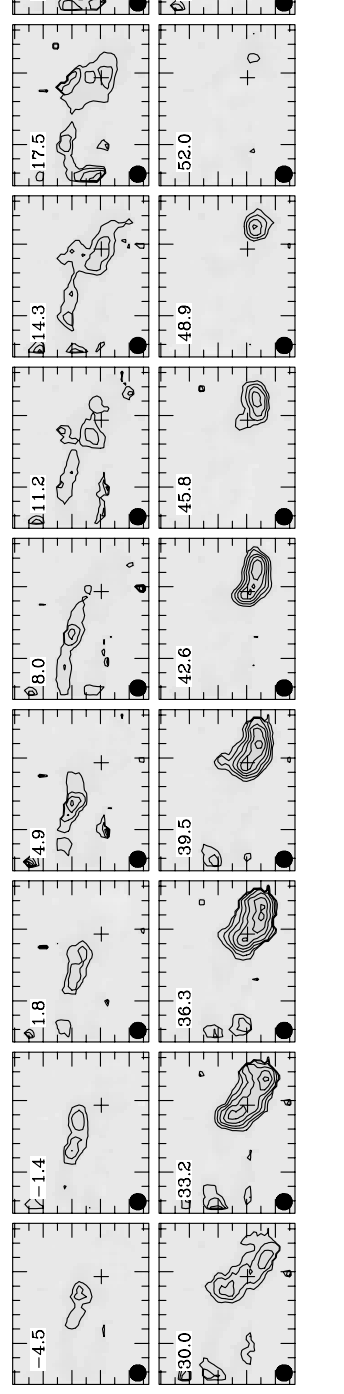

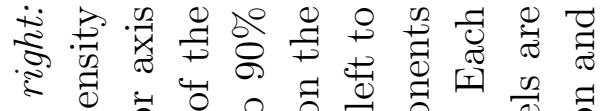

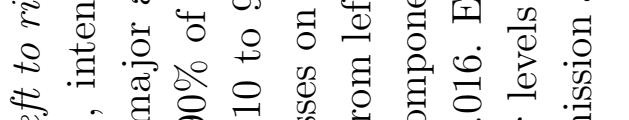

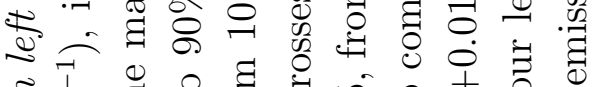

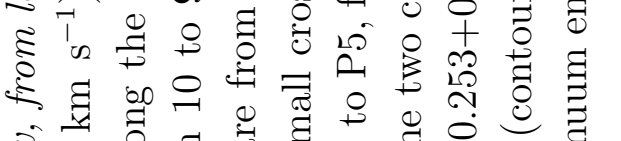

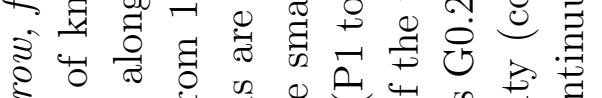

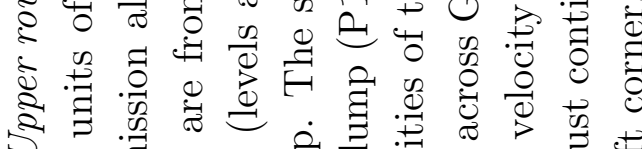

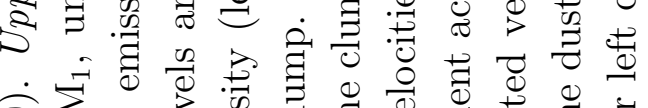
O

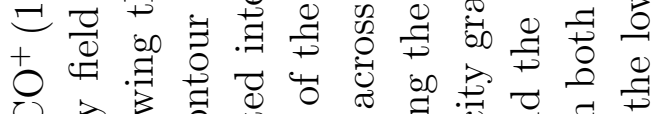

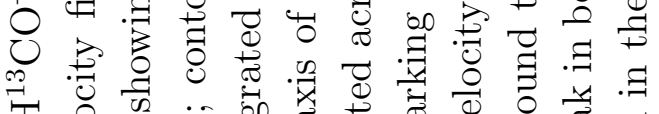
击 क्ष

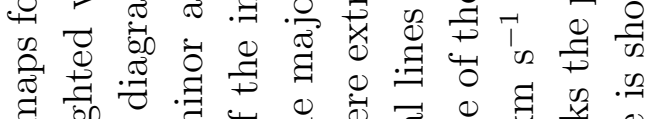

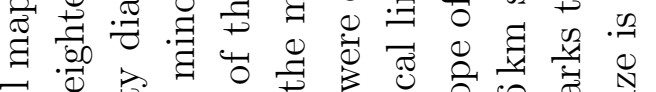

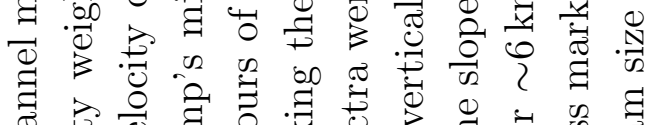

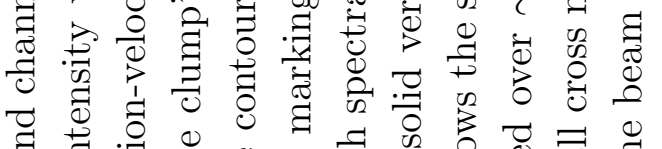

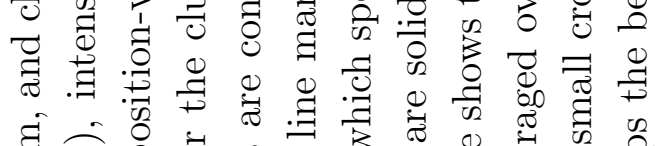

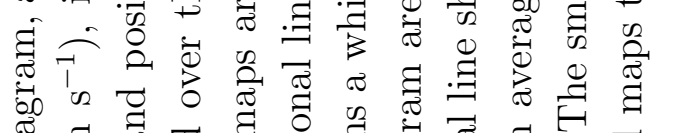

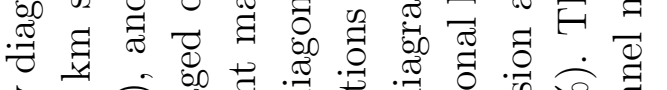

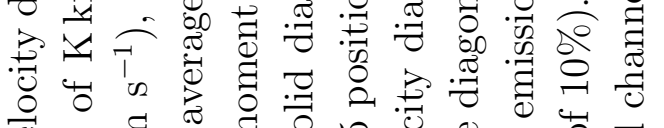

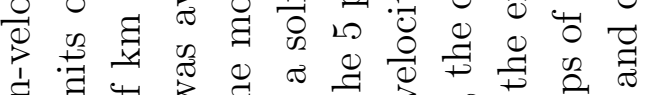

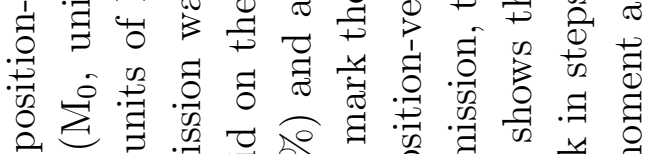

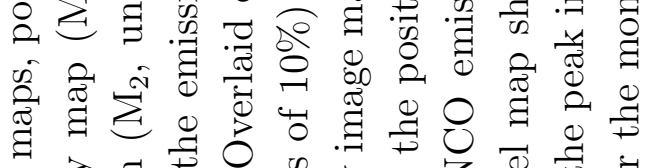

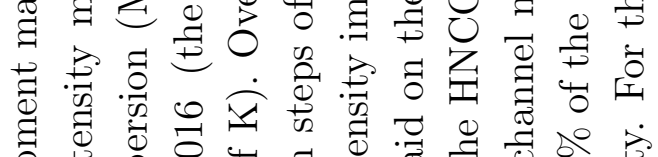

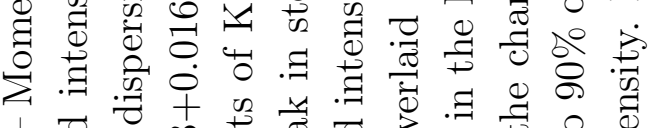

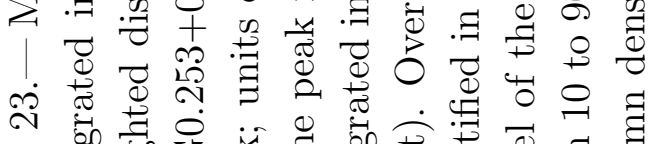

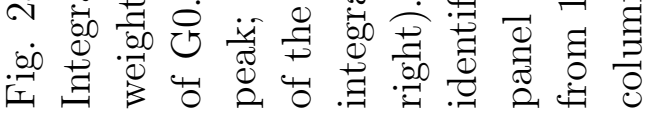



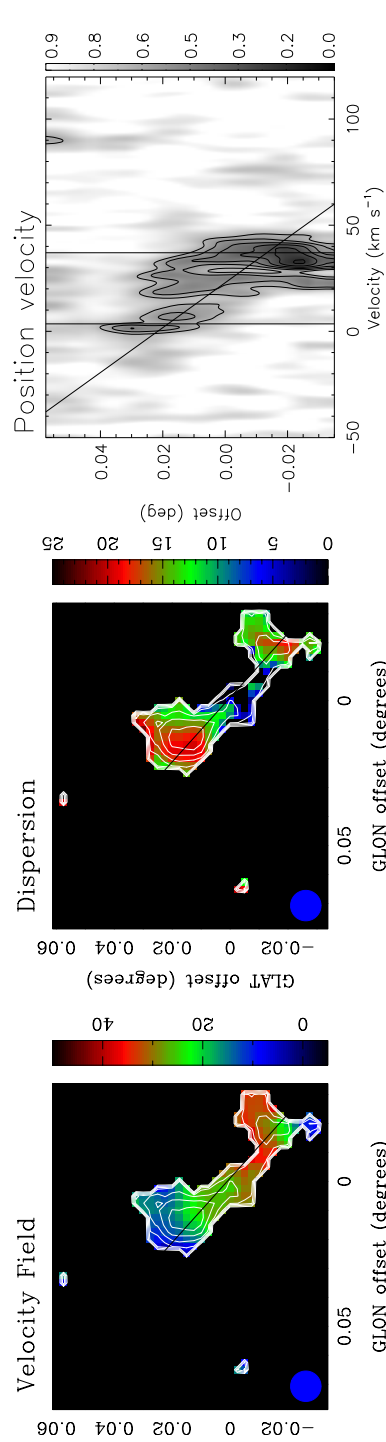

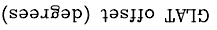

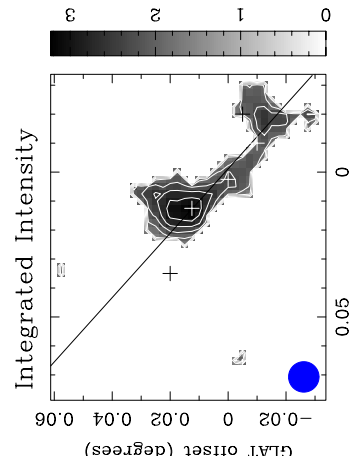

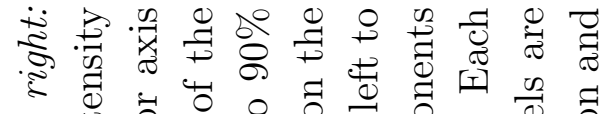

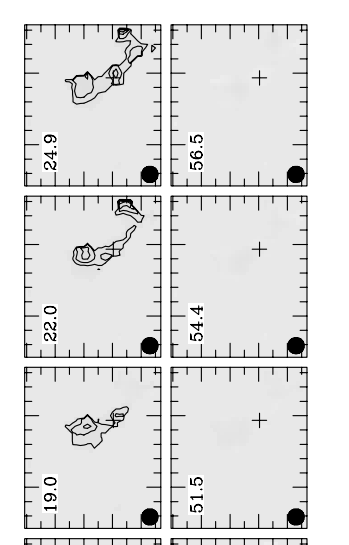

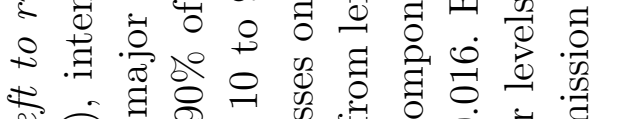

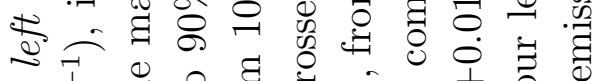

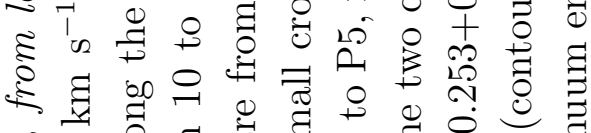

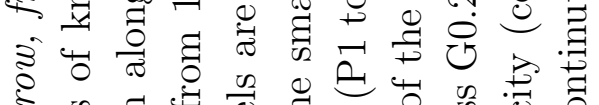

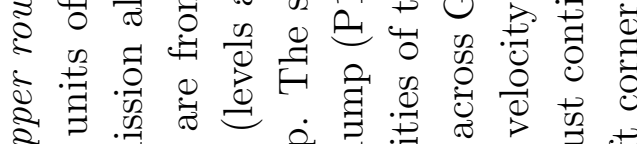

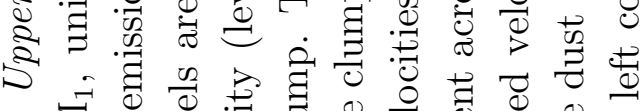

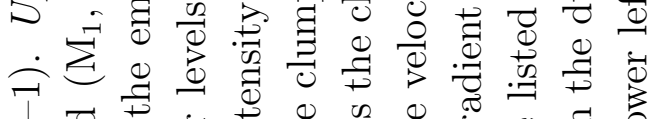

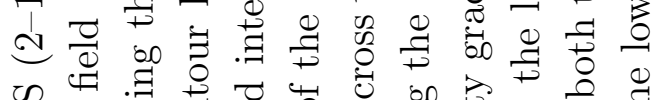

记

离

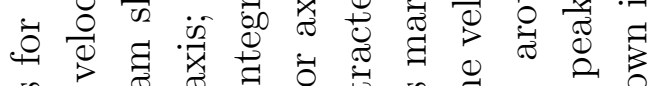

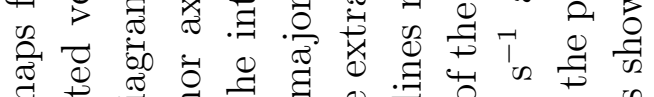

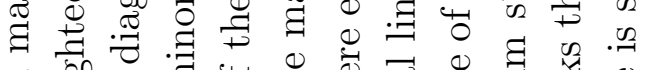

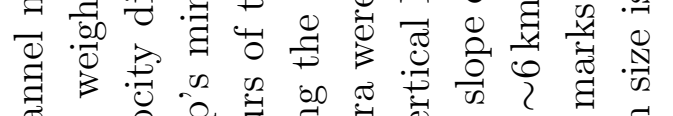

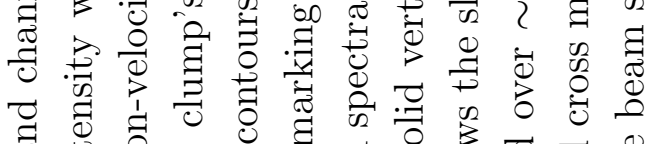

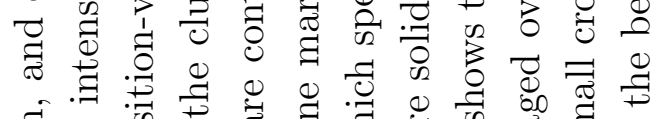

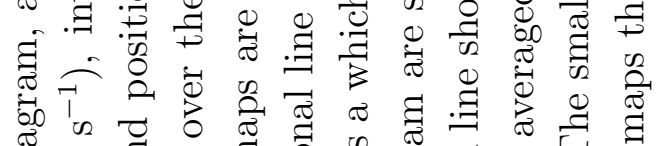

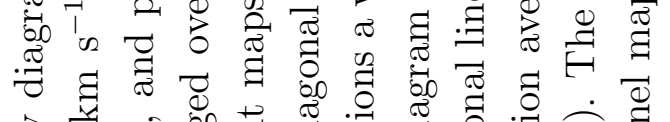

焉

范

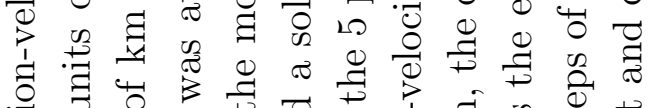

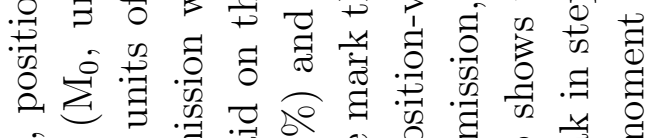

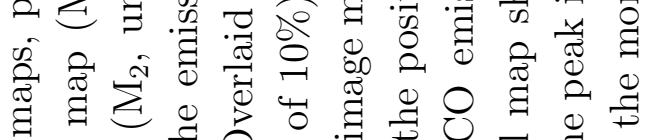

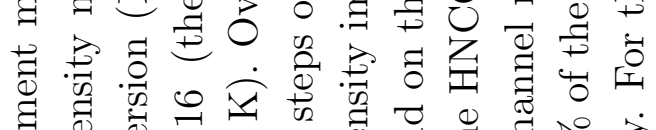

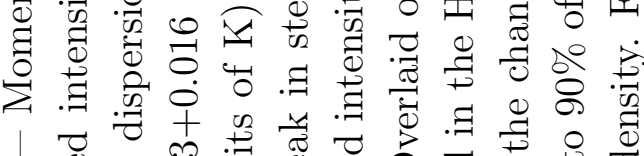

।.

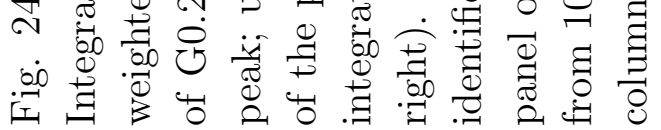



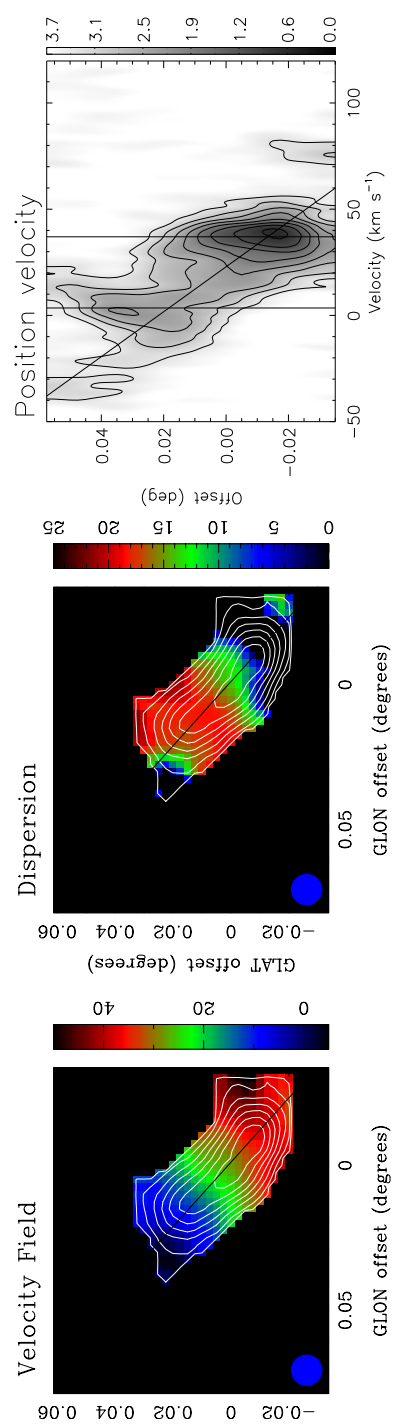

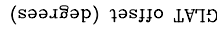

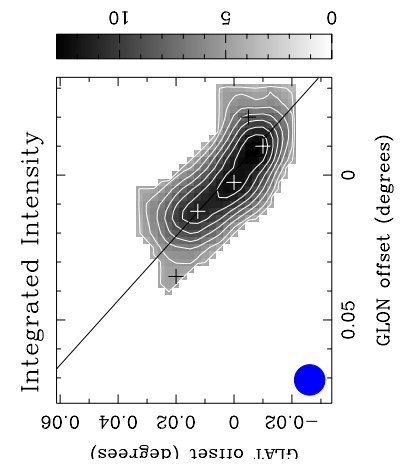

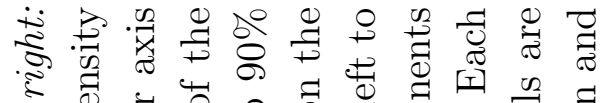
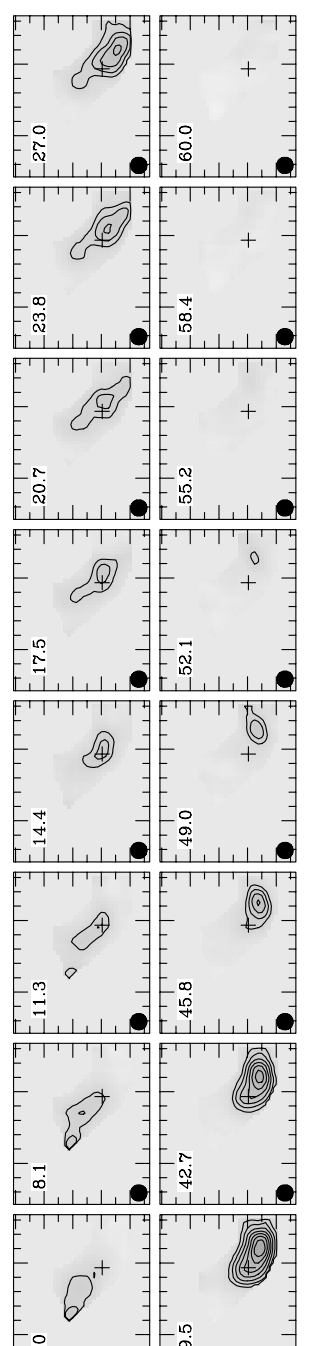

is
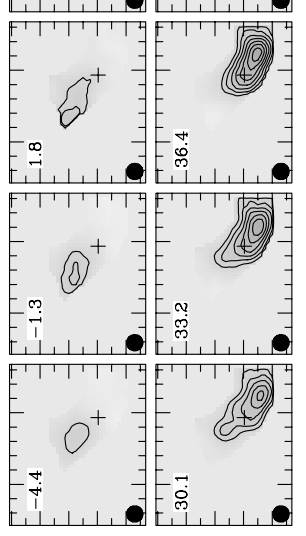

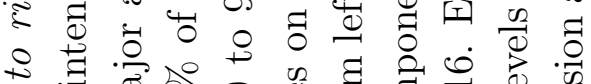

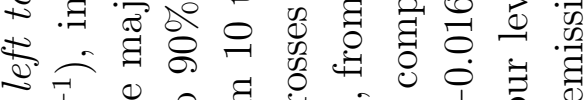
is

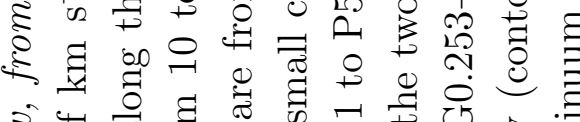

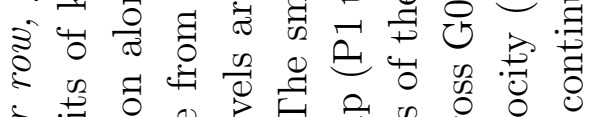

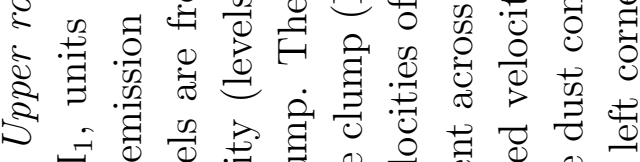

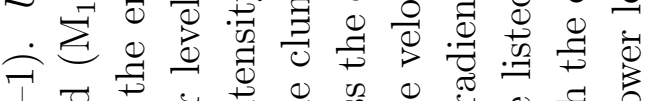

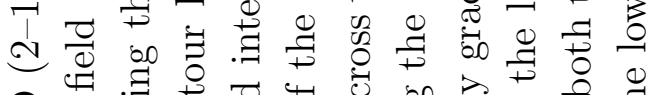

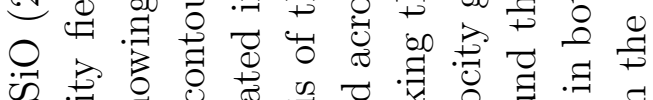

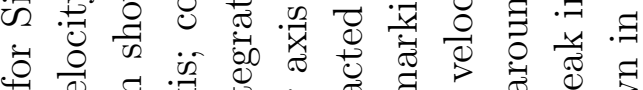

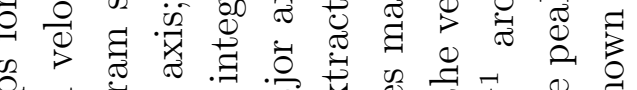

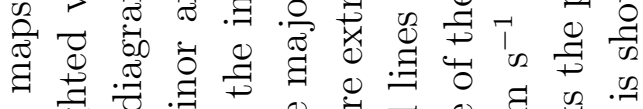

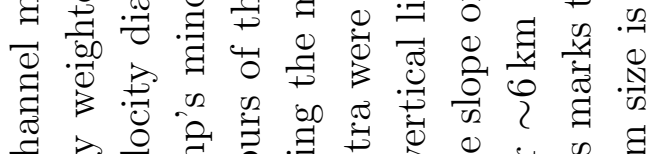

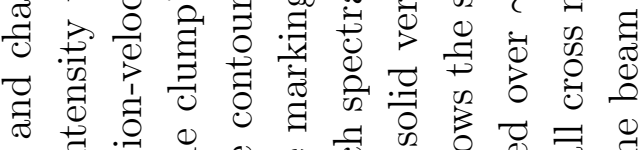

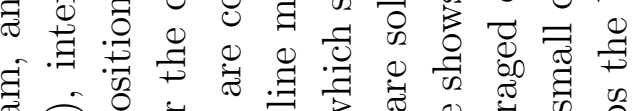

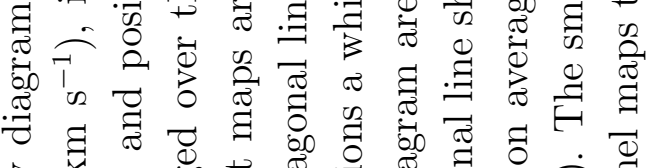

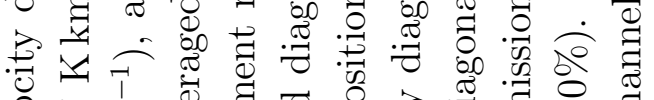

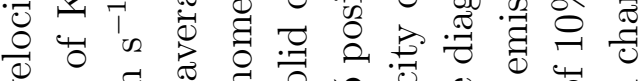

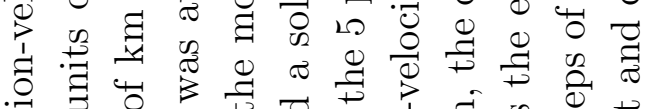

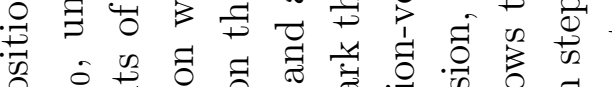

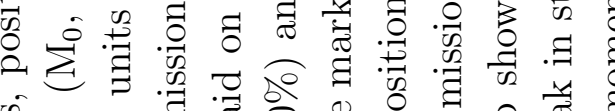
w

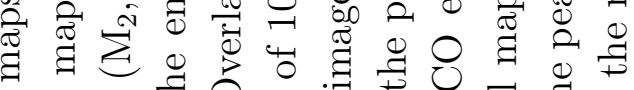

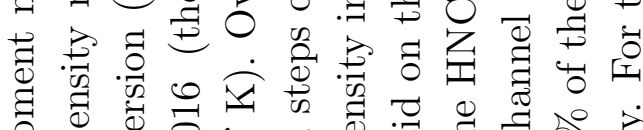

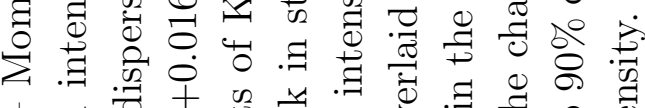

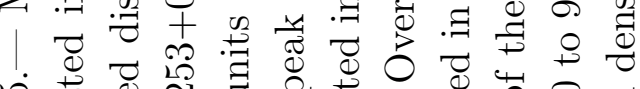

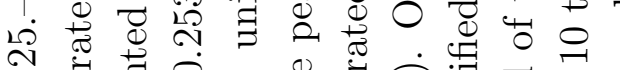

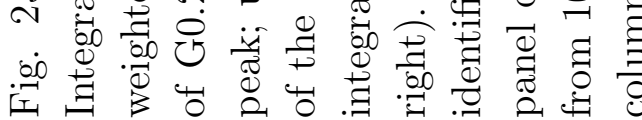



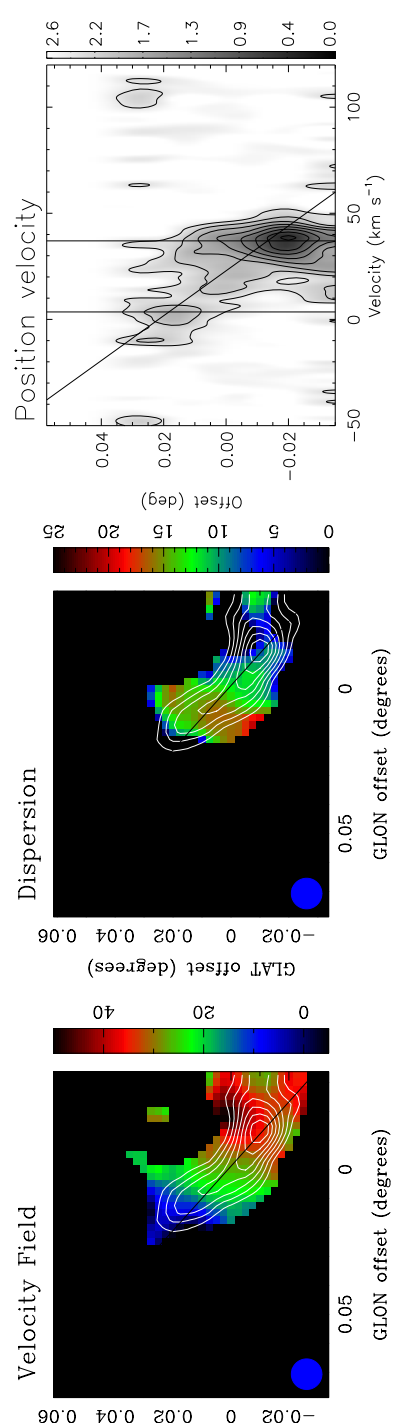

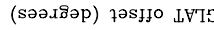

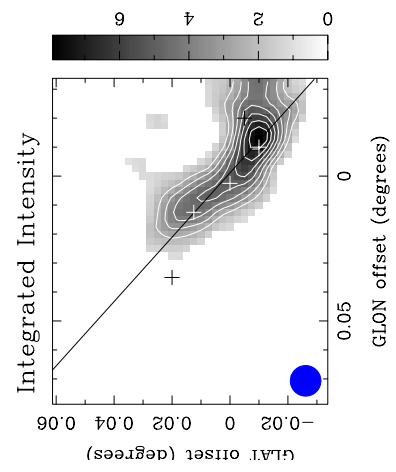

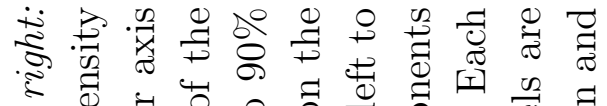
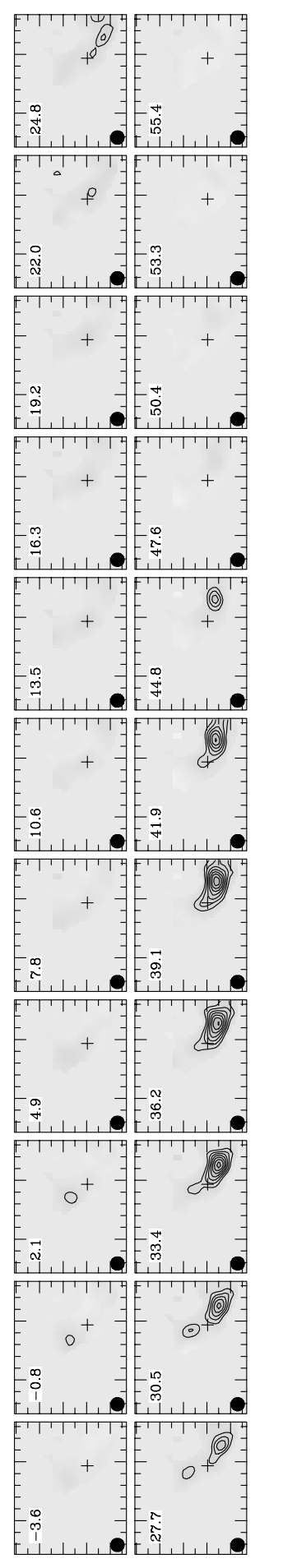

究 光

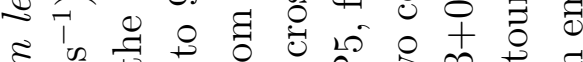

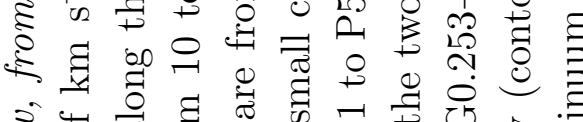

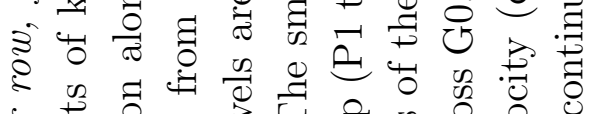

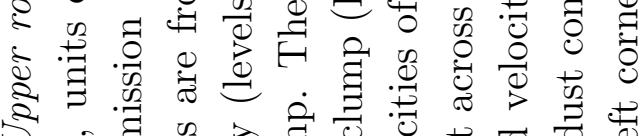

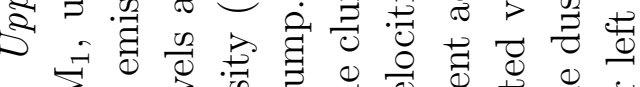

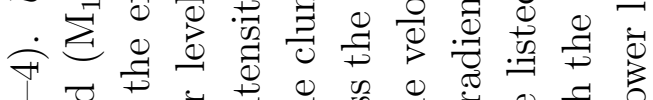

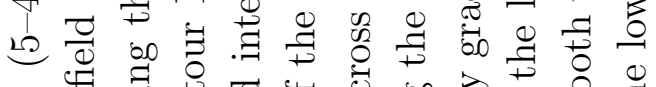

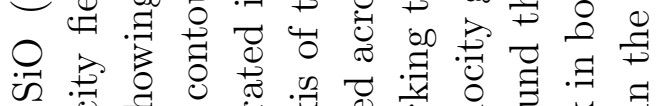

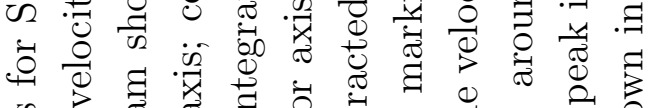

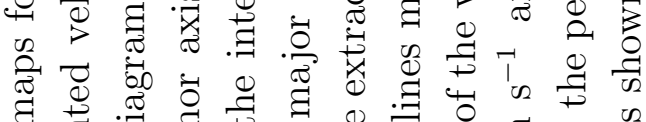

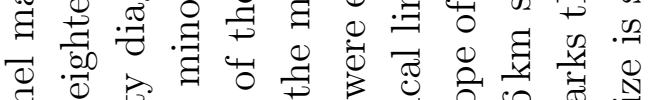

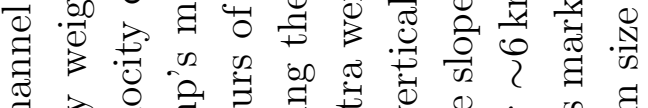

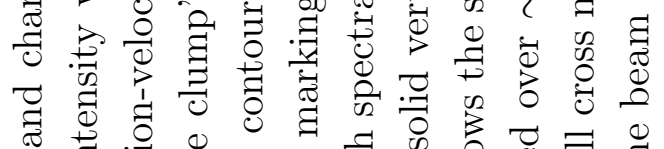

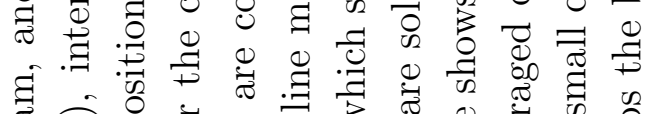

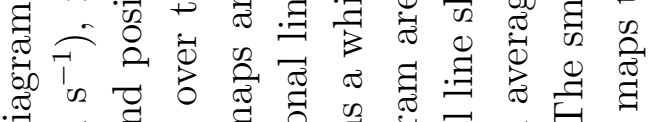

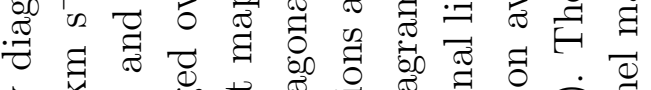

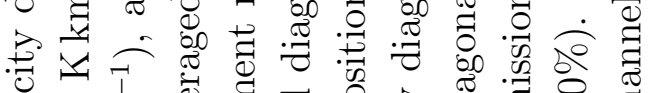

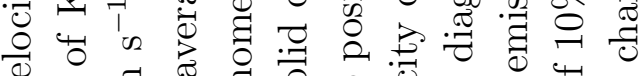

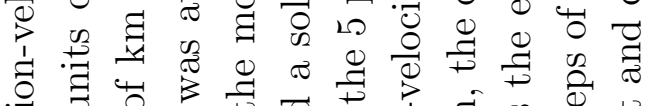

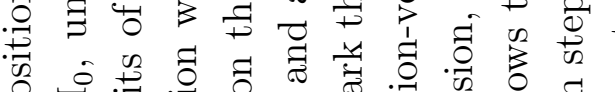

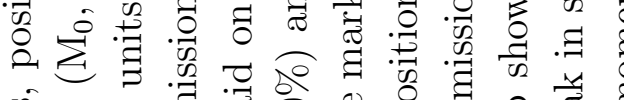
के की

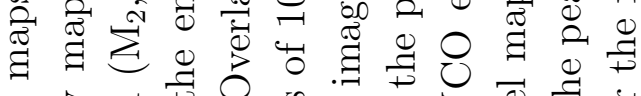

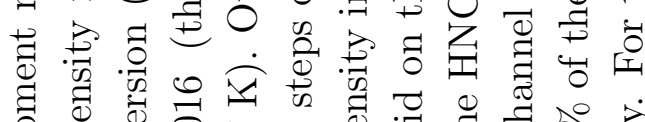

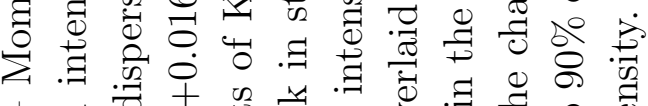

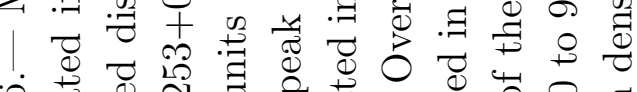
ஸे

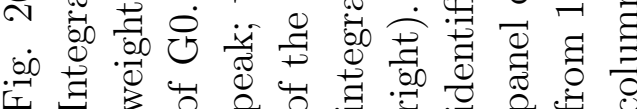



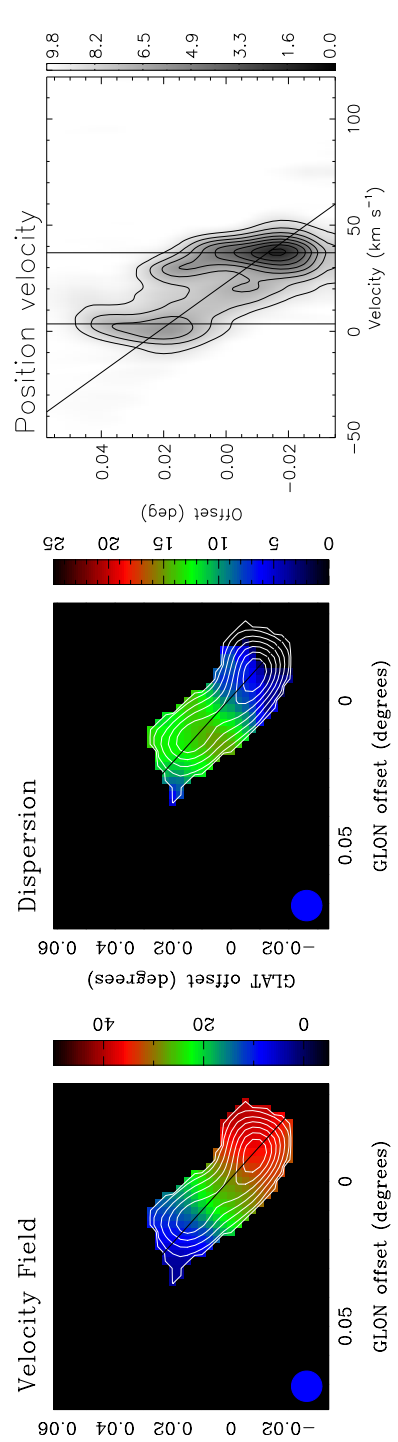

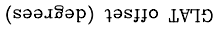



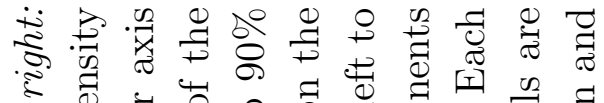
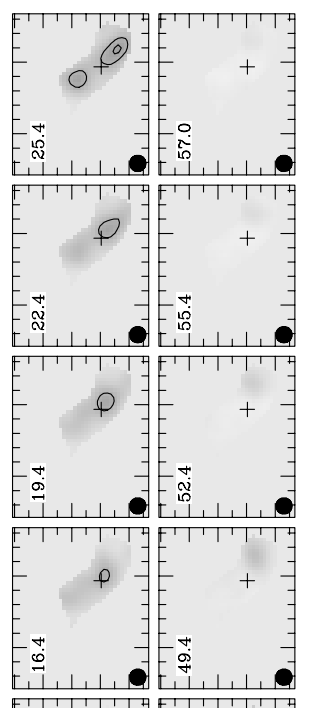

0
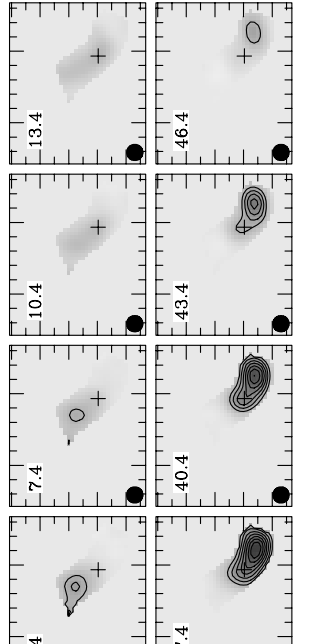

$+$
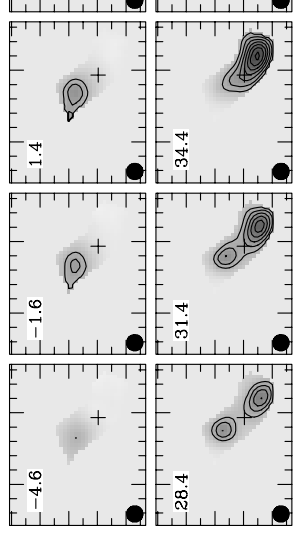

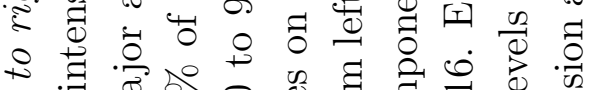
岕 ह ।

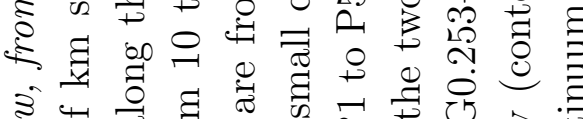

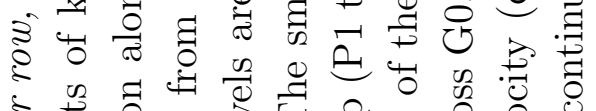

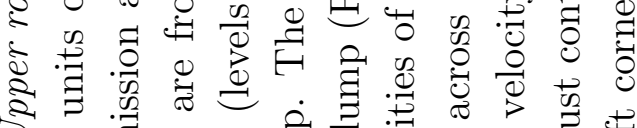

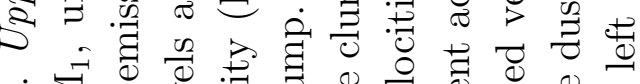

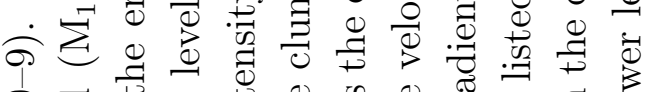

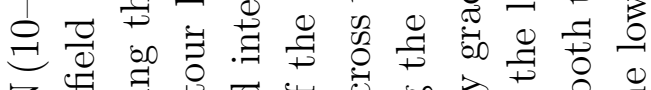
乙

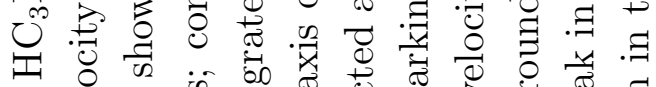
苛 कै

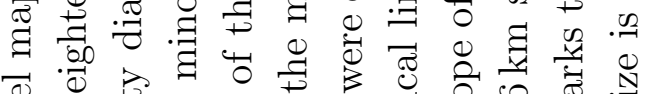

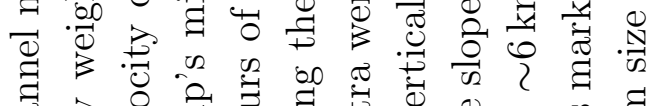

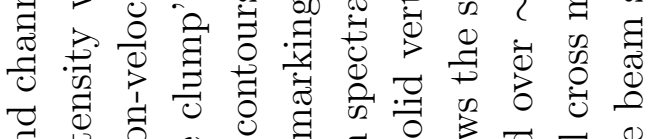

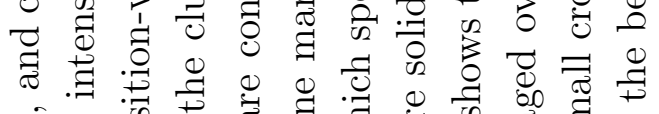

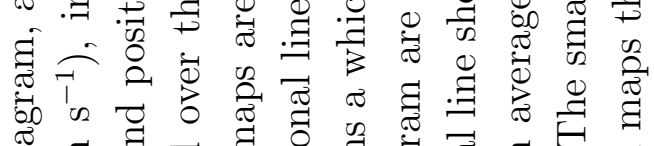
胥 :

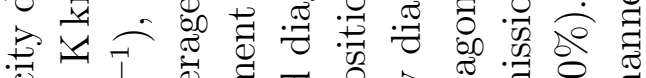

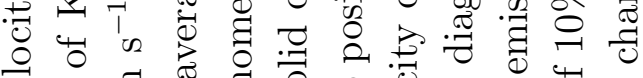

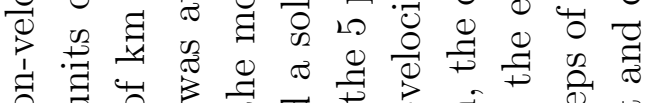

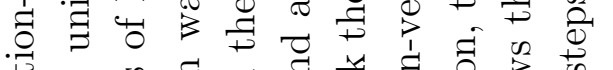

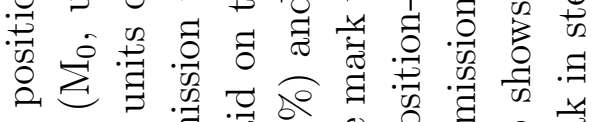

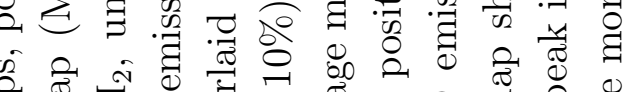

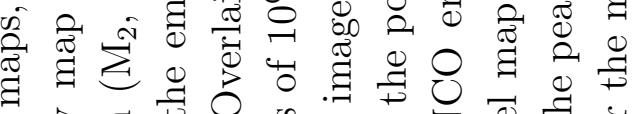

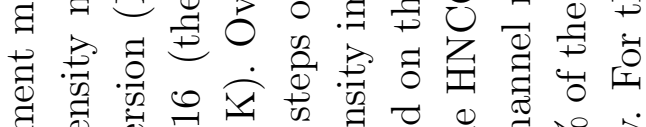

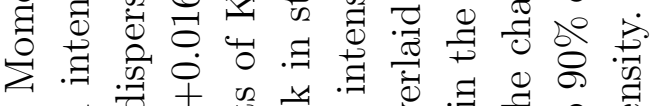

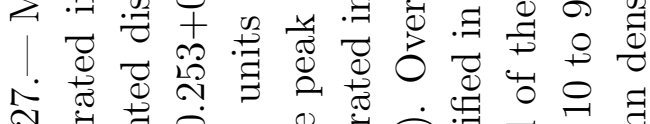

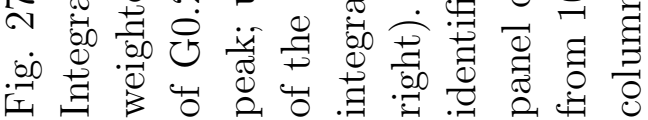



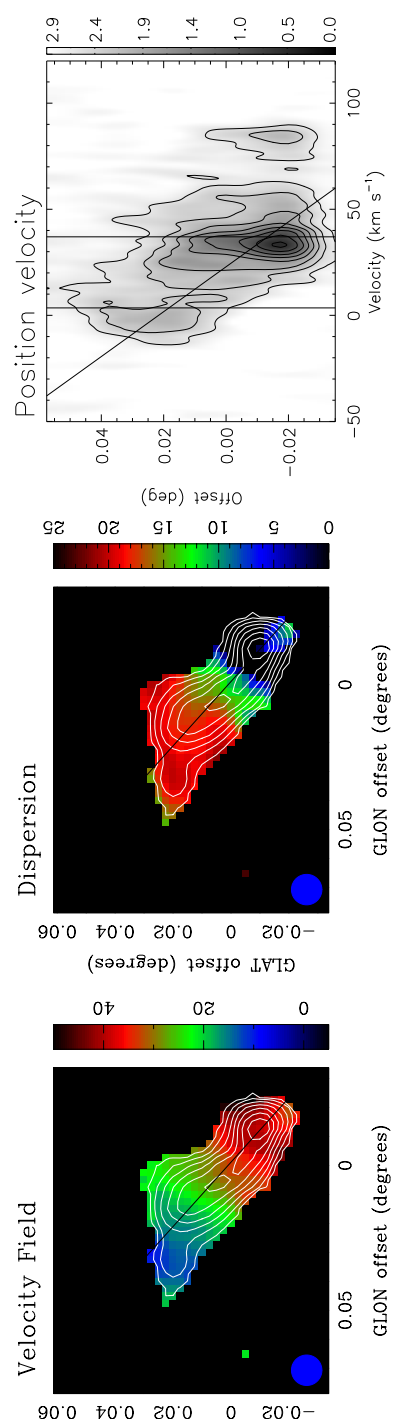

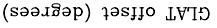

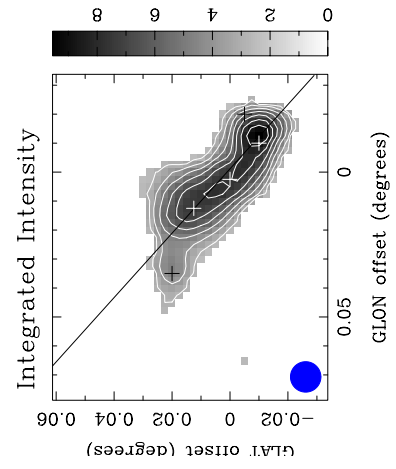

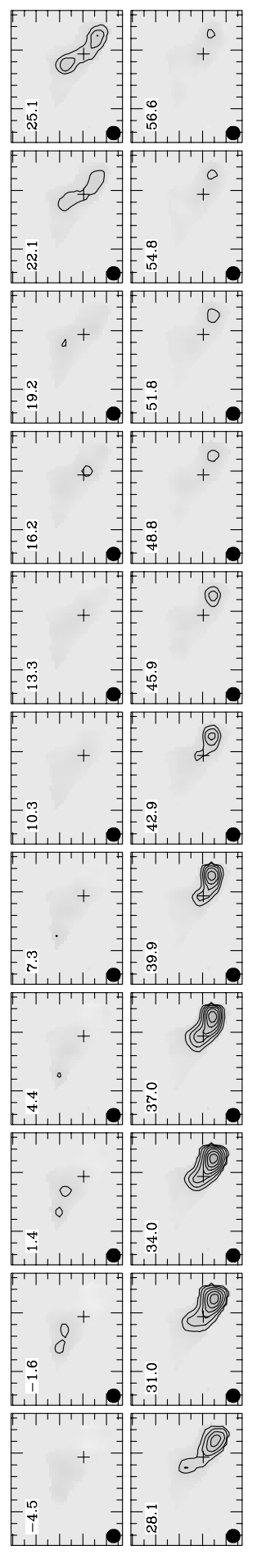

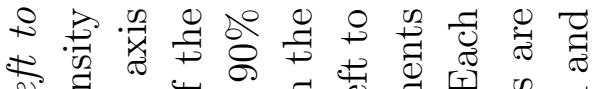
હ है.

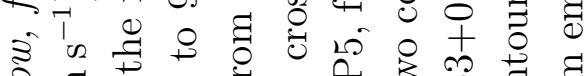
○ घ

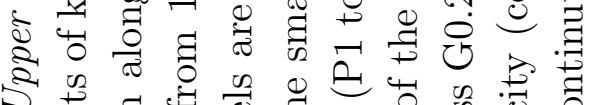
S. W :

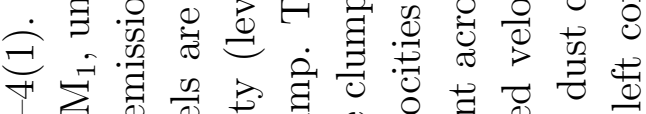

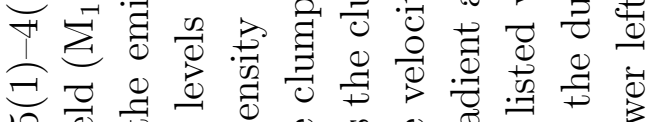

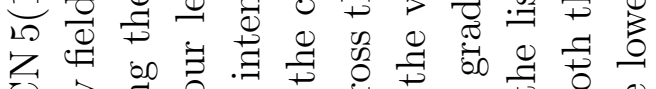

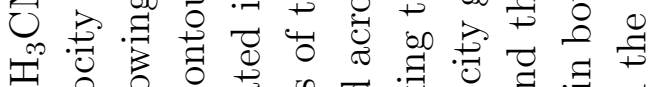

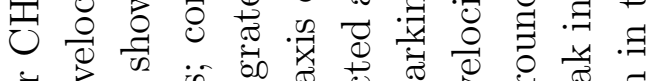

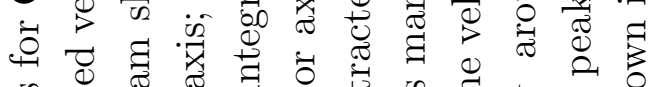

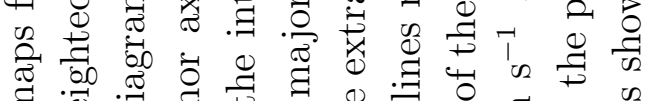

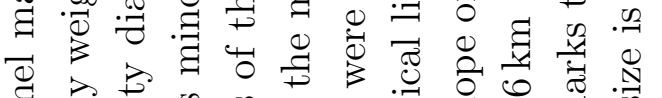

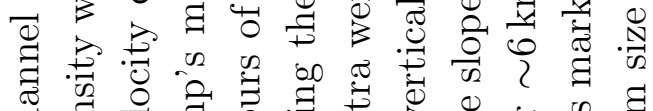

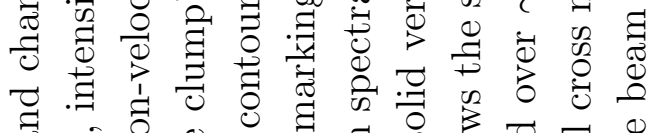
茫年.

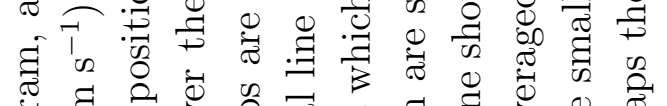

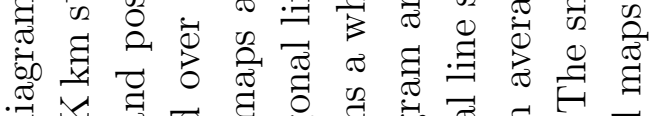
㞼 1 व 㐘

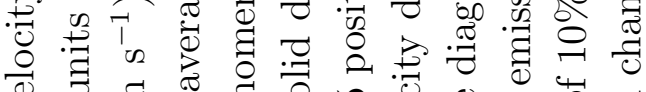

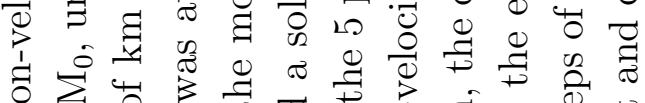

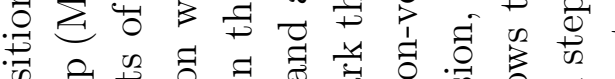

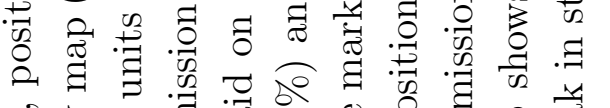

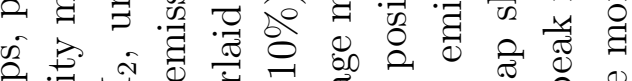

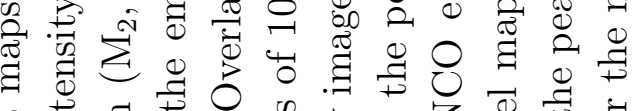

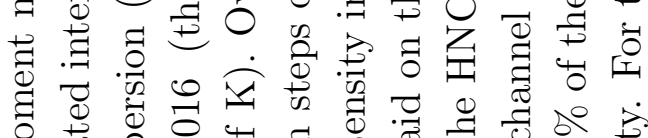

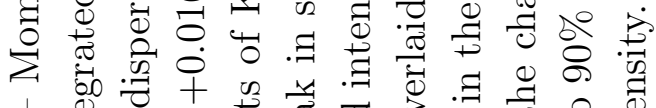

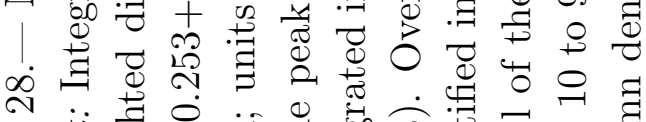

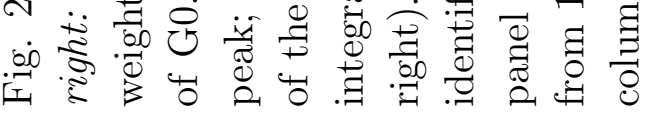



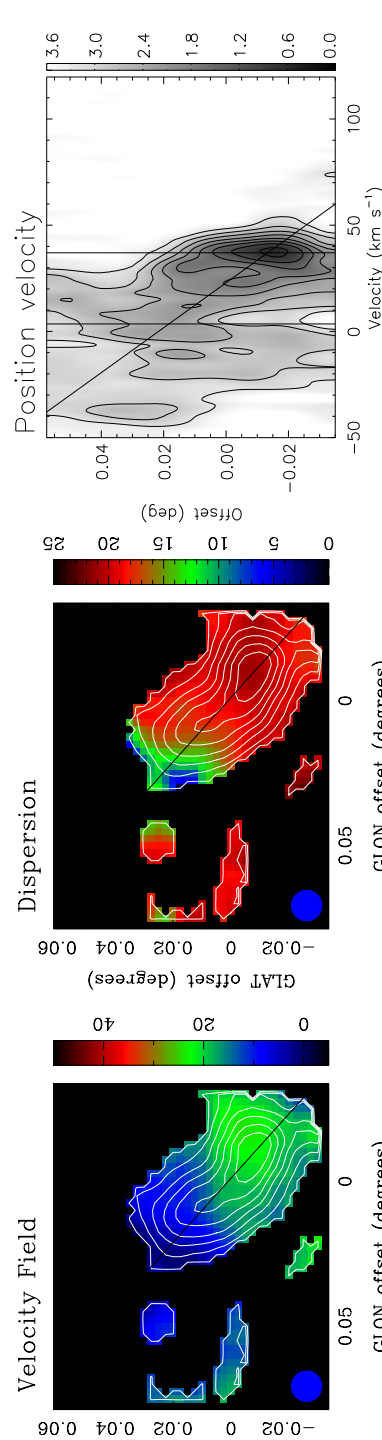

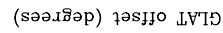
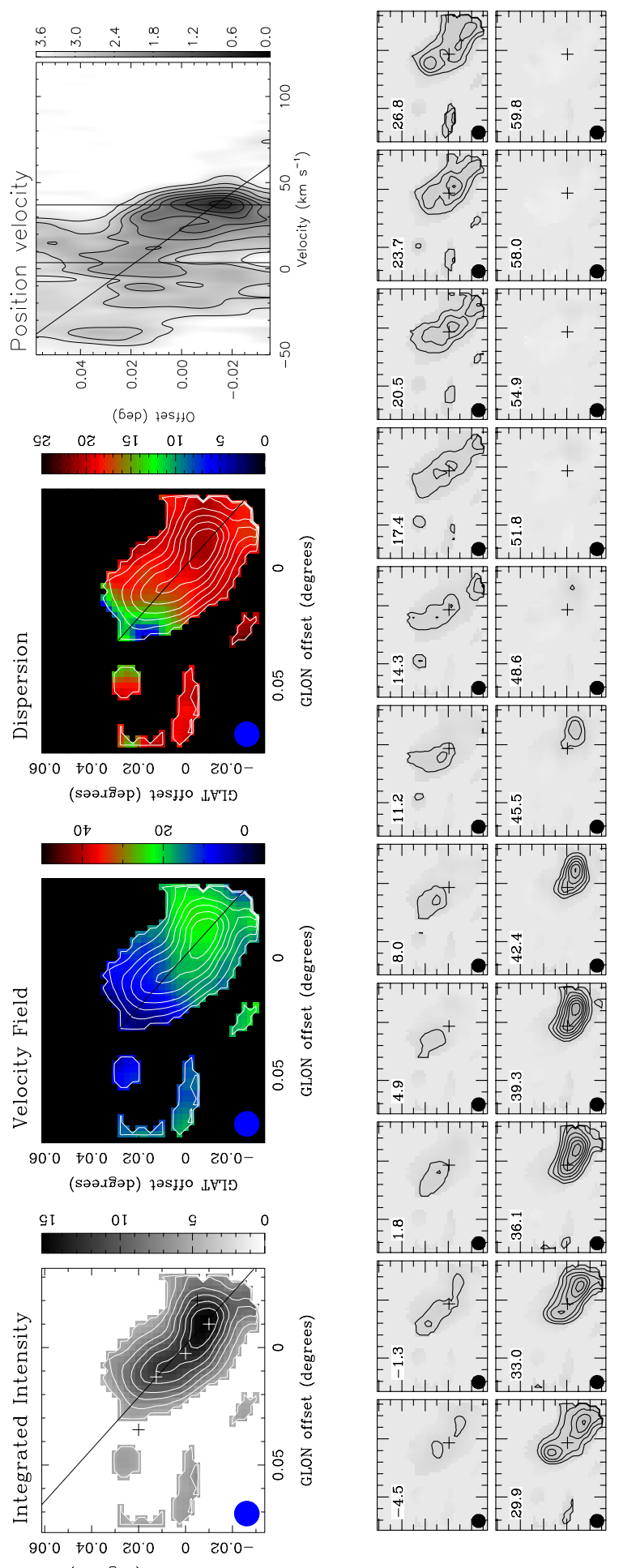

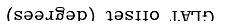

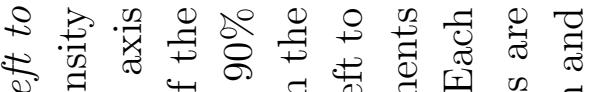

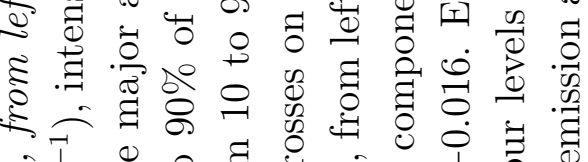
इं। ¿ ఏँ

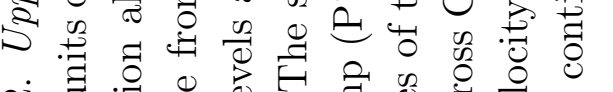

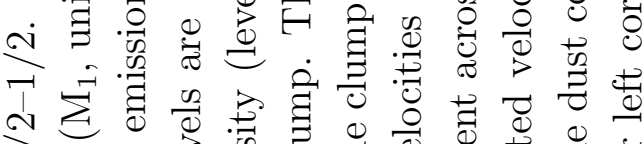

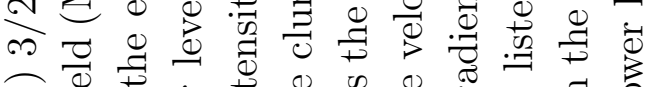
○ी

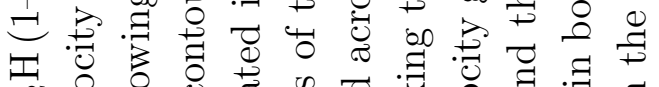

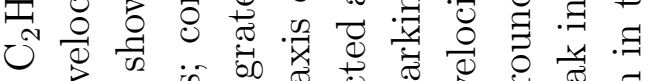

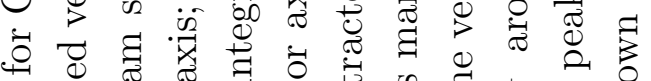

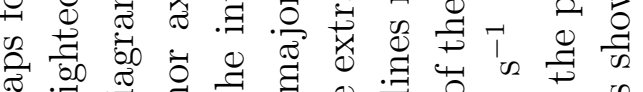

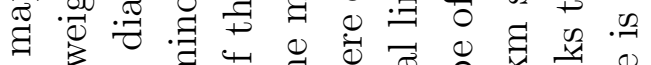

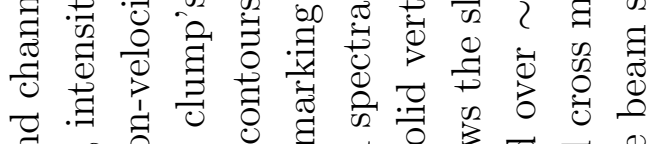
范

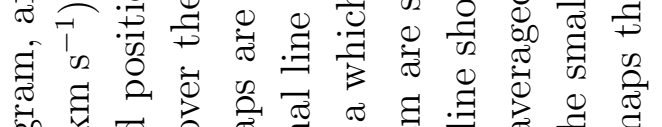

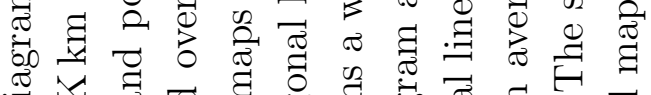
坖 焉 荡

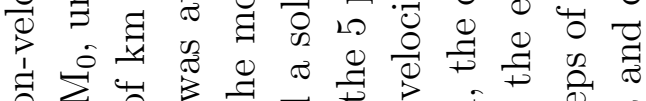

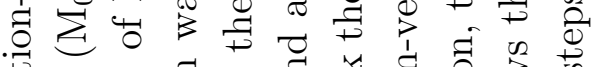

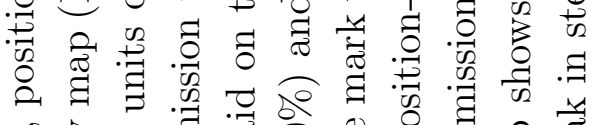

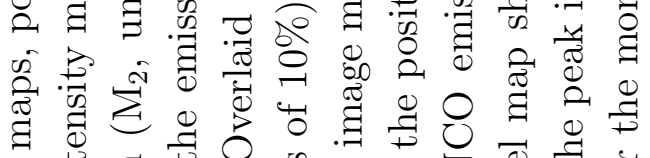

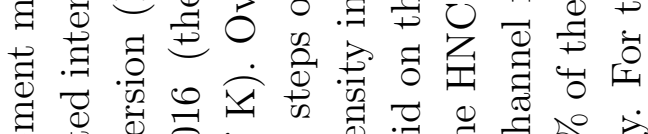

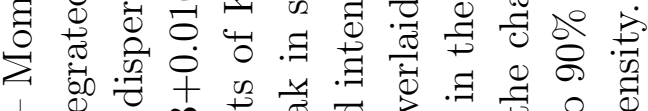

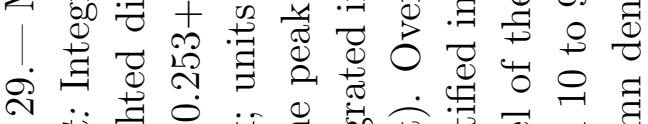

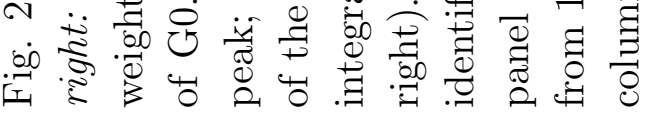

